\title{
UNITARY REPRESENTATIONS OF LIE GROUPS WITH COCOMPACT RADICAL AND APPLICATIONS
}

\author{
BY \\ L. PUKANSZKY ( $\left.{ }^{1}\right)$
}

\begin{abstract}
The paper gives a necessary and sufficient condition in order that a connected and simply connnected Lie group with cocompact radical be of type I. This result is then applied to a characterization of Lie groups, all irreducible unitary representations of which are completely continuous.
\end{abstract}

\section{INTRODUCTION}

Let $G$ be a connected Lie group. In a previous paper [20] we established a canonical bijection between the underlying set of the primitive ideal space Prim( $(G)$ of the group $C^{*}$ algebra $C^{*}(G)$ of $G$ and the set of all characters of $G$ or, equivalently, the set of all quasi-equivalence classes of normal representations of $G$ (cf. loc. cit., Theorem 1, p. 119). Here we say that a factor representation $T$ of $G$ is normal (cf. loc. cit., p. 106) if it generates a semifinite factor such that the range of $T$, viewed as a representation of $C^{*}(G)$, contains a nonzero traceable operator with respect to a normal trace on the former. If so, the image, by virtue of the quoted bijection, of the quasi-equivalence class of $T$ is equal to the kernel of $T$. We recall also that $G$ is of type I if and only if any of its irreducible unitary representations is normal. In this, and only in this, case our bijection gives rise to one between $\operatorname{Prim}(G)$ and $\hat{G}$ (= set of all unitary equivalence classes of irreducible unitary representations of $G$ ). This being so, the question of the concrete construction of the normal representations of $G$ and of a corresponding description, in terms of $G$ and of its Lie algebra $g$, of $\operatorname{Prim}(G)$ poses itself in a natural fashion. If $G$ is solvable this has been completely settled (cf. loc. cit., Theorem 3, p. 134). Extending earlier investigations by L. Auslander and B. Kostant (cf. [1]), here our theory associates with each $J$ in $\operatorname{Prim}(G)$ a well-determined geometrical object $\theta(J)$ ("generalized orbit"; cf. [18, Chapter II, p. 512]), constructed by aid of the coadjoint representation of $G$ acting on $\mathfrak{g}^{\prime}$ (= dual of the underlying space of g), and provides an algorithm to obtain any normal $T$ with $\operatorname{ker}(T)=J$ via

Received by the editors April 22, 1976.

AMS (MOS) subject classifications (1970). Primary 22D25; Secondary 46L05, 46L25.

(')This paper was partially supported by a grant from the National Science Foundation. The results of this paper were announced in [21].

- American Mathematical Society 1978 
$\theta(J)$. If the structure of $G$ is sufficiently simple, e.g. if it is nilpotent, the family of all generalized orbits coincides with the orbit space of the coadjoint representation, introduced in this context first by A. A. Kirillov. One further question is, in what way the properties of $J$ in $\operatorname{Prim}(G)$ of being of type I or CCR are reflected by $\theta(J)$. In the solvable case the first problem has already been answered (cf. [18, Theorem 2, p. 551]), but as to the second, only partial results are available (cf. [16, Theorem 2, p. 111]), [17, Theorem, p. 465], and further below).

The purpose of the present paper is to initiate an extension of these investigations to arbitrary connected and simply connected Lie groups; we do this here under the assumption that the radical of $g$ is cocompact. A new feature, when compared with the solvable case, consists in the fact, otherwise fully expected from special cases, that only a part of $g^{\prime}$ is involved in the construction of the characters (cf. II.2.1). We start by setting up, in general in a many-to-one fashion, a map from $\operatorname{Prim}(G)$ onto a family of geometrical objects constructed by the coadjoint representation of $G$. This permits us to derive criteria in order that a primitive ideal be of type I (cf. Proposition 2), which is the principal result of this paper. In this manner, in particular, we obtain a characterization, in terms of the relevant part of $g^{\prime} / G$, of the type I groups of our class (cf. Theorem 1). This generalizes the analogous result for the solvable case, obtained by Auslander and Kostant (cf. Corollary loc. cit.). In order to indicate the nature of the principle according to which the "relevant" part of $\mathfrak{g}^{\prime}$ is singled out, we give some details for the special case when the radical is nilpotent (cf. Proposition 1); this is of a certain independent interest. These results are used in an essential fashion in the proof of Proposition 2. If some slight additional condition is fulfilled, the "relevant" elements are those $g \in g^{\prime}$, for which there is a character $\chi_{g}$ of the connected component of the identity $\left(G_{g}\right)_{0}$ of the stabilizer $G_{g}$ of $g$ in $G$, such that $d x_{g}=i\left(g \mid g_{g}\right)$, where $g_{g}$ is the subalgebra of $g$ corresponding to $\left(G_{g}\right)_{0}$. Let us denote by $W(G)$ the set of all these elements. Then there is a map, uniquely determined by some natural conditions (cf. II.2.3, Remark 2), from $\hat{G}$ onto $W(G) / G$ which, if $G$ is nilpotent, reduces to the classical Kirillov bijection between $\hat{G}$ and $g^{\prime} / G$. In the general case the number, always finite, of the elements of $\hat{G}$, corresponding to the same $O=W(G) / G$, is to be determined as follows. If $O=G g$, let us set $\dot{G}_{g}=\operatorname{ker}\left(\chi_{g}\right)$ and assume first that $\dot{G}_{g} \subsetneq\left(G_{g}\right)_{0}$. The exact sequence

$$
1 \rightarrow\left(G_{g}\right)_{0} / \dot{G}_{g} \rightarrow G_{g} / \dot{G}_{g} \rightarrow G_{g} /\left(G_{g}\right)_{0} \rightarrow 1
$$

defines a central extension by the circle group of the finite group $G_{8} /\left(G_{g}\right)_{0}=$ $\pi_{1}(O)$. This being so, the number in question is equal to the number of all irreducible representations of $G_{g} / \dot{G}_{g}$ which, on the circle group, reduce to 
multiples of the identity map of the latter onto itself. If $\dot{G}_{g}=\left(G_{g}\right)_{0}$ take the number of elements in the dual of $G_{g} /\left(G_{g}\right)_{0}$. Conversely, given a central extension, by the circle group, of an arbitrary finite group, there is a group $G$ of our class and a $g \in W(G)$ such that the above sequence is isomorphic to the given one.

In Chapter III we apply the results of Chapter II to obtain a description of all connected and simply connected CCR Lie groups, In spite of some artificiality in the question, we believe that the answer offered here can serve to further illustrate the amount of information one can derive from the orbit picture. We recall that $G$ admits the quoted property if, given a right invariant Haar measure $d a$ on $G$, for any irreducible representation $T$ and for any continuous function $\varphi$ of compact support the operator $\int_{G} \varphi(a) \cdot T(a) \cdot d a$ is completely continuous. This has been known for some time to be the case if $G$ is either semisimple (Harish-Chandra) or nilpotent (J. Dixmier). On the other hand, among solvable Lie groups already the affine group of the real line fails to have this property. Combining the results quoted above of Auslander and Kostant with those of C. C. Moore (cf. [2, Chapter VD), one can state in this case simple necessary and sufficient conditions in terms of $\mathrm{g}^{\prime} / G$, in order that $G$ be CCR (cf. Corollary, III.3 below); we shall do the same for a general $G$ (cf. loc. cit. Theorem 2). Other classes of groups have been investigated with respect to the CCR property by R. Lipsman (cf. [12]). If $G$ is CCR then, in particular, $\operatorname{Prim}(G)$ must be a $T_{1}$ space. The latter property can be expressed equivalently in terms of several others (cf. Proposition 3); part of this has been obtained independently by C. C. Moore and J. Rosenberg (cf. [14]). In particular, the condition that the radical be cocompact practically imposes itself in this context (cf. loc. cit. (i) $\Leftrightarrow$ (iv)). Another of these properties is provided by the concept of a generalized completely continuous representation. This is a normal representation, $T$ say, such that $T\left(C^{*}(G)\right)$ is composed of operators which are traceable with respect to some normal trace. If $T$ is irreducible, it admits this property if and only if $T\left(C^{*}(G)\right)$ is composed of completely continuous operators in the classical sense. To say that $G$ is $C C R$ is equivalent to requiring that it be of type I and that any of its normal representations be a generalized completely continuous one. In this manner Theorem 2, providing a description of all such groups in terms of $g^{\prime} / G$, will be derived by combining Theorem 1 and Proposition 3, which give analogous characterizations, respectively, for each of these properties.

Observe that the first two sections of Chapter III leading to the proof of Proposition 3 are essentially independent of Chapter II.

The reader is assumed to be familiar with the basic results of $\mathbf{G}$. W. Mackey's theory of induced representations (cf. [13]), which we shall often 
use without special reference. General assumptions and notational conventions are collected in the Appendix. We shall refer to it by $\mathbf{A}+$ number of the relevant section.

\section{UNITARY REPRESENTATIONS OF LIE GROUPS WITH A COCOMPACT RADICAL}

The principal result of this chapter is Proposition 2, which provides a characterization in terms of the orbits of the coadjoint representation of the primitive ideals of type I of a connected Lie group, the Lie algebra of which satisfies the condition in the title of this paper. In the first section we have collected some preparatory material drawn mostly from the paper [8] of $\mathbf{M}$. Duflo. In $\$ 2$ we first give the definition of admissibility (cf. 2.1), which singles out the orbits relevant for the representation theory. Next (cf. 2.3), confining ourselves to groups with cocompact and nilpotent radical, we exhibit a canonical surjection from the dual of our group onto the collection of admissible orbits (cf. Lemma 6 and Proposition 1 loc. cit.); in the nilpotent case this map coincides with the Kirillov isomorphism. A primitive ideal $J$ is of type $I$ if and only if the action of our group on a space, depending on $J$, is smooth, and if a certain integer associated with $J$ is finite (cf. [20, Proposition 3 , (1) and (2), respectively, p. 1111). We find the expression in terms of the coadjoint representation of these conditions, respectively, in the last two sections.

1. In this section $G$ can be an arbitrary connected Lie group; we denote its Lie algebra by $g$.

1.1.(a) For the details of the constructions, due to $M$. Duflo, sketched in the sequel, cf. $[8, \mathrm{p} .106]$. Let $n$ be the nilradical of $g$ and $f$ a nonzero element, which we keep fixed, of $\mathfrak{n}^{\prime}$ (= dual of the underlying space of $\mathfrak{n}$ ). Let us set $\mathfrak{b}=\mathfrak{n} / \mathfrak{n}_{f}$ (A.1); $\mathfrak{b}$ carries a nondegenerate skewsymmetric bilinear form $B$ obtained, through passage to quotient, from the map $\left(l_{1}, l_{2}\right) \rightarrow\left(\left[l_{1}, l_{2}\right], f\right)$ $\left(l_{1}, l_{2} \in \mathfrak{n}\right)$. If $\operatorname{dim}(\mathfrak{b})>0$, since $\pi_{1}(\operatorname{Sp}(B))$ is isomorphic to an infinite cyclic group, $\operatorname{Sp}(B)$ admits a well-determined double covering which we denote by $\operatorname{Mp}(B)$. If $\operatorname{dim}(b)=0$ the latter will stand for the cyclic group of order two. We write $\tau$ for the canonical morphism of $\mathrm{Mp}(B)$ onto $\operatorname{Sp}(B)$ and $\varepsilon$ for the element, different from the unity, of $\mathrm{Mp}(B)$ for which $\tau(\varepsilon)$ is equal to the unity of $\operatorname{Sp}(B)$. If $a \in G_{f}$ we denote by $j(a)$ the operator, corresponding to $\operatorname{Ad}(a) \mid \mathfrak{n}$, on $\mathfrak{v}=\mathfrak{n} / \mathfrak{n}_{f}$; clearly we have $j(a) \in \operatorname{Sp}(B)$. This being so, we define $\mathcal{G}$ as the subgroup of $B_{f} \times \operatorname{Mp}(B)$ composed of all pairs $\left(a, a^{\prime}\right)$ for which $j(a)=\tau\left(a^{\prime}\right)$. Putting $p\left(\left(a, a^{\prime}\right)\right)=a,(\mathcal{S}, p)$ is a twofold covering of $G_{f}$.

(b) Given $a \in \mathcal{G}$ and $n \in N(N=\exp (\mathfrak{n}) \subset G$; cf. A.4), we put $a(n)=$ $p(a) n(p(a))^{-1}$, and define the semidirect product of topological groups $M=\mathcal{G} \times{ }_{j} N$ through the law of composition $(a, n)(b, m)=\left(a b, b^{-1}(n) m\right)$. 
Let $\pi$ be a concrete irreducible unitary representation of $N$ belonging, in the sense of Kirillov, to the orbit $N f \subset \mathfrak{n}^{\prime}$ (cf. e.g. [18, pp. 494-495]). The following facts are fundamental for the rest of this paper (cf. [8, Theorème 6.1, p. 107]; for some details also see the proof of Lemma 1 below). There is a canonical choice of a unitary representation $W$ of $M$ on $H(\pi)$ (cf. A.2) such that: (1) $W \mid N=\pi$ (2) Let us put $\Re={ }_{p}^{-1}\left(N_{f}\right)$ and write $\varphi_{f}$ for the character of $\Re$ determined by $d \varphi_{f}=i\left(f \mid n_{f}\right)$ and $\varphi_{f}(\varepsilon)=-1$. Then if $V=W \mid \mathcal{S}$,

$$
V(n) \equiv \overline{\varphi_{f}(n)} \pi(p(n)) \quad(n \in \Re)
$$

(cf. loc. cit., Lemme 5.2 and Lemme 5.5, pp. 104-105).

(c) In the following we reproduce a classical construction, due to Mackey, in a manner adapted to the context of (d) below. For $\pi \in \hat{N}$ we denote by the same letter a concrete representation of this class, and by $\mathscr{F}$ the set of all projective extensions of $\pi$ to $G_{\pi}$ (for the notions employed, cf. e.g. [2, Chapter I, §4, p. 18]). (1) Let $\rho$ be an element of $\mathscr{F}$ and $\alpha$ the corresponding extension cocycle. We write $\mathbb{S}(\delta)$ for the group defined on the set $G_{\pi} \times \mathrm{T}(\mathrm{T}=$ circle group) by the law of composition $(a, u)(b, v)=(a b, \alpha(a, b) u v)\left(a, b \in G_{\pi}\right.$; $u, v \in \mathrm{T})$. We define the unitary representation $\tilde{\rho}$ of $\&(\rho)$ by $\tilde{\rho}((a, u)) \equiv$ $\rho(a) u$. We also write $\mathrm{T}$ for $\{(e, u) ; u \in \mathrm{T}\} \subset \circlearrowleft(\rho)(e=$ unity). Finally, let $q$ be the projection onto $G_{\pi}$. (2) Assume $\rho_{j} \in \mathscr{F}$; we put $\mathscr{G}_{j}=\mathbb{B}\left(\rho_{j}\right)$ and distinguish notions, relative to $\rho_{j}$, by the corresponding subscript $(j=1,2)$. There is a unique measurable map $g: G_{z} \rightarrow \mathrm{T}$ such that $\rho_{2}(a) \equiv \overline{g(a)} \rho_{1}(a)$ $\left(a \in G_{n}\right)$; putting

$$
(\delta g)(a, b) \equiv g(a b) / g(a) g(b) \quad\left(a, b \in G_{\pi}\right),
$$

we have then $\alpha_{2}=\delta g \cdot \alpha_{1}$. This being so, there is an isomorphism $\varphi_{21}$ of $\mathbb{S}_{1}$ onto $\mathbb{G}_{2}$ uniquely determined by the properties: $(\alpha) \varphi_{21} \mid \mathrm{T}$ is the identity map onto $\mathrm{T} \subset \mathbb{S}_{2} ;(\beta) q_{2} \circ \varphi_{21}=q_{1} ;(\gamma) \tilde{\rho}_{2} \circ \varphi_{21}=\tilde{\rho}_{1}$. One sees at once that with the previous notations we have $\varphi_{21}((a, u)) \equiv(a, g(a) u)$. Observe that if $\rho_{j} \in$ $\mathcal{F}(j=1,2,3)$, evidently $\varphi_{31}=\varphi_{32}{ }^{\circ} \varphi_{21}$. (3) Let $H$ be the direct product of groups $\Pi_{\rho \in \mathcal{F}}\left(\mathcal{S}(\rho)\right.$, and $p(\rho)$ the projection $H \rightarrow \mathbb{S}(\rho)$. Given $\rho_{j} \in \mathscr{F}$ we write $p_{j}=p\left(p_{j}\right)(j=1,2)$. We denote by $G_{\pi}^{e}$ the set of all elements $s$ of $H$ for which $\varphi_{21}\left(p_{1}(s)\right)=p_{2}(s)$ for all $\left(\rho_{1}, \rho_{2}\right) \in \mathscr{F} \times \mathscr{F} . G_{\pi}^{e}$ is a subgroup of $H$ and, for any $\rho \in \mathscr{F}, q(\rho)=p(\rho) \mid G_{\pi}^{e}$ is an isomorphism of $G_{\pi}^{e}$ onto $\mathbb{S}(\rho)$. (4) We recall (cf. loc. cit., p. 24) that $\&(\rho)$ carries the structure of a locally compact group uniquely determined by the condition that the product of a Haar measure on $G_{\pi}$ and on T, respectively, be of the same kind on $B(\rho)$. We endow $G_{\pi}^{e}$ with a similar structure by requiring that $q(\rho)$ be an isomorphism of topological groups. Observe that this definition is independent of the choice of $\rho \in \mathscr{F}$. In fact, if $q_{j}=q\left(\rho_{j}\right)\left(\rho_{j} \in \mathscr{F}\right.$ for $\left.j=1,2\right)$, then $q_{2} \circ \stackrel{-1}{q}_{1}=\varphi_{21}$ is clearly a Borel isomorphism $\&\left(\rho_{1}\right) \rightarrow \circlearrowleft\left(\rho_{2}\right)$, and thus also an isomorphism of the underlying topological groups (cf. loc. cit., Proposition 3.3, p. 14). (5) Again let $\alpha$ be the 
extension cocycle corresponding to $\rho \in \mathscr{F}$. Since $\alpha(a m, b n) \equiv \alpha(a, b)(a, b$ $\in G_{\pi}^{\mathbf{e}} ; m, n \in N$ ), and the projection $(a, u) \mapsto a$ is continuous (cf. loc. cit., p. 24), the set $\{(n, u) ; n \in N, u \in T\}$ is a closed invariant subgroup of \& $(\rho)$. We also have, with the previous notations, $\varphi_{21}((n, u)) \equiv(n, u) \in \mathbb{S}\left(\rho_{2}\right)$, and hence there is a unique injection $i: N \times \mathrm{T} \rightarrow G_{\pi}^{e}$ such that $q(\rho) \circ i$ is the identity map for all $\rho \in \mathscr{F}$. Below we shall of ten simply write $N$ for $i(N) \subset G_{\pi}^{e}$. In this manner, if $\Phi$ is the canonical homomorphism from $G_{\pi}^{e}$ onto $\mathfrak{M}=G_{q}^{e} / N$, we have the exact sequence

$$
1 \rightarrow N \stackrel{i}{\rightarrow} G_{\pi}^{e} \rightarrow \mathfrak{M} \rightarrow 1 .
$$

(d) Let $f \in \mathfrak{n}^{\prime}$ and $\pi \in \hat{N}$ be as in (b). We recall that $G_{\pi}=G_{f} \cdot N$ (cf. e.g. [18, Lemma 5.1, p. 497]). We denote by $\pi^{\prime} \in \mathcal{F}$ a projective extension of $\pi$ to $G_{\pi}$ (cf. A.2) and by $\alpha$ the corresponding cocycle. Since for any $a \in \mathcal{G}$ and $n \in N$ we have

$$
V(a) \pi(n) V\left(a^{-1}\right)=\pi(a(n))=\pi^{\prime}(p(a)) \pi(n)\left(\pi^{\prime}(p(a))\right)^{*},
$$

there is a unique measurable map $k: \mathcal{G} \rightarrow \mathrm{T}$ (= circle group) such that

$$
V(a) \equiv k(a) \pi^{\prime}(p(a)) \quad(a \in \mathbb{S}) .
$$

One verifies at once that $\alpha(p(a), p(b)) \equiv(\delta k)(a, b)(a, b \in \mathcal{G})$ and therefore, since $k(\varepsilon)=-1$ (cf. (b)), the map $\eta: \mathcal{G} \rightarrow G\left(\pi^{\prime}\right)$ defined by $\eta(a)=$ $(p(a), k(a))$ is an injective homomorphism. If $\rho_{j} \in \mathscr{F}$ and $\eta_{j}$ is analogous to $\eta$ above $(j=1,2)$, we have $\eta_{2}=\varphi_{21} \circ \eta_{1}$. Hence there is an injective homomorphism $\gamma: \mathcal{G} \rightarrow G_{\pi}^{e}$ uniquely determined by the condition that $q(\rho) \circ \gamma=\eta$ for all $\rho \in \mathcal{F}$. Putting $\mathscr{\mathcal { C }}=\operatorname{ker}\left(\varphi_{f}\right)$ (cf. (b)), we are going to show that $\operatorname{ker}(\Phi \circ \gamma)=\dot{\gamma}$. To this end it is enough to observe that if $k$ and $\pi^{\prime}$ are as above, by $V(n) \equiv \overline{\varphi_{f}(n)} \pi(p(n))(n \in \Re)$ (cf. (b) end) we have $k(n) \equiv \overline{\varphi_{f}(n)}$. Therefore the conditions $p(a) \in N$ and $k(a)=1(a \in \mathcal{G})$ are equivalent to $a \in \mathfrak{\Upsilon}$. Let us define finally $h: G_{\pi}^{e} \rightarrow G_{\pi}$ by $h(s)=a$ if $q(\rho)(s)=(a, u) \in \mathbb{S}(\rho)(\rho \in \mathcal{F})$. Denoting by $j$ the canonical homomorphism $\mathcal{G} \rightarrow \mathcal{G} / \dot{\pi}$, by $\gamma(\mathcal{G}) N=G_{\pi}^{e}$ we have the diagram

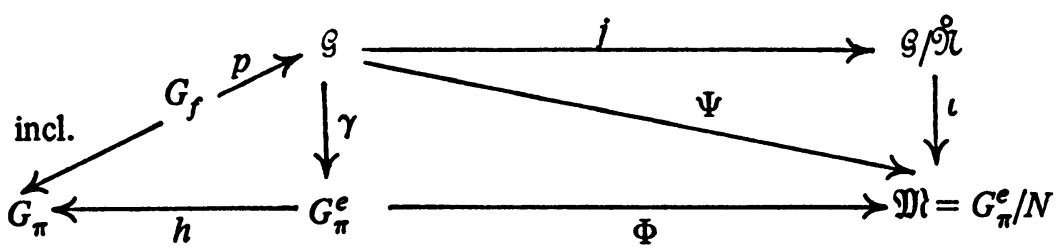

where $i$ is an isomorphism onto and incl. signifies inclusion.

(e) Later we shall make repeated use of the following obvious implication of the previous considerations. Let us assume that $G$ is contained as closed invariant subgroup in the (say) connected Lie group (S), and suppose $a \in G_{f}$. 
$\mathcal{G}$ and $G_{\pi}^{e}$ being contained as invariant subgroups in the corresponding objects constructed with respect to $\mathbb{S}, a$ induces the automorphism $\zeta^{\prime}$ of $\mathcal{G}$ and $\eta^{\prime}$ of $G_{\pi}^{e}$. Clearly $\zeta^{\prime}(\stackrel{\Upsilon}{ })=\vartheta^{\circ}$ and $\eta^{\prime}(N)=N$, and thus we can define automorphisms $\zeta$ and $\eta$ of $\mathfrak{M}$ such that $\zeta \circ \Psi=\zeta^{\prime}$ and $\eta \circ \Phi=\eta^{\prime}$. This being so, we have $\zeta=\eta$.

1.2. In the following we shall consider $\mathfrak{g}, \mathfrak{n}$ and their complexifications as left $\operatorname{Aut}(G)$ modules via the adjoint representation, and $g^{\prime}, \mathfrak{n}^{\prime}$, etc. as $\operatorname{Aut}(G)$ modules with respect to the contragredient action. Let $\alpha$ be some fixed element of $\operatorname{Aut}(G)$. Suppose that $\pi_{1}$ is an irreducible unitary representation of $N$ corresponding to the orbit $N f_{1} \subset \mathfrak{n}^{\prime}$; then if $f_{2}=\alpha f_{1}, \pi_{2}=\alpha \pi_{1}$ (A.3) will belong to $N f_{2} \subset \mathfrak{n}^{\prime}$ (cf. e.g. [18, p. 497, Remark 4.1]). Let $M_{j}$, $W_{j}$ be the objects belonging to $f_{j}$ and $\pi_{j}(j=1,2)$ as introduced in 1.1(b).

LEMMA 1. With the above notations there is a canonical isomorphism $\beta$ of $M_{1}$ onto $M_{2}$ such that $W_{2}=\beta W_{1}$.

Proof. (a) We start by defining an isomorphism $\beta$ of $\mathcal{G}_{1}$ onto $\mathcal{G}_{2}$; for unexplained notations cf. 1.1(a) above. Let us write $G_{j}, \mathfrak{n}_{j}$ for $G_{f}$ and $\mathfrak{n}_{f}$ if $f=f_{j}$ respectively $(j=1,2)$. We have, evidently, $\alpha\left(G_{1}\right)=G_{2}, \alpha(\mathfrak{n})=\mathfrak{n}$, and hence also $\alpha\left(\mathfrak{n}_{1}\right)=\mathfrak{n}_{2}$. If $\operatorname{dim}\left(\mathfrak{b}_{j}\right)=0$, by definition $\mathcal{S}_{j}$ is direct product of $G_{j}$ and of a cyclic group of order $2(j=1,2)$. If $\left(a, a^{\prime}\right) \in \mathcal{G}_{1}$ we define $\beta\left(\left(a, a^{\prime}\right)\right)=\left(\alpha(a), a^{\prime}\right)$. Assume next that $\operatorname{dim}\left(\mathfrak{y}_{j}\right)>0$. By going to the quotients we obtain from $\mathfrak{n} \ni l \mapsto \alpha l$ an isomorphism $w$ from $\mathfrak{b}_{1}=\mathfrak{n} / \mathfrak{n}_{1}$ onto $\mathfrak{v}_{2}=\mathfrak{n} / \mathfrak{n}_{2}$, which makes $B_{1}$ and $B_{2}$ correspond to each other, and thus gives rise to an isomorphism $i: \operatorname{Sp}\left(B_{1}\right) \rightarrow \operatorname{Sp}\left(B_{2}\right)$. Since $\pi_{1}\left(\operatorname{Sp}\left(B_{j}\right)\right) \sim Z(j=1,2)$, there is a well-determined isomorphism $v: \operatorname{Mp}\left(B_{1}\right) \rightarrow \operatorname{Mp}\left(B_{2}\right)$ such that $i \circ \tau_{1}=\tau_{2} \circ v$. Since clearly $i \circ j_{1}=j_{2} \circ\left(\alpha \mid G_{1}\right)$, if $\left(a, a^{\prime}\right) \in \mathcal{G}_{1}$ (which by definition is equivalent to $\left.j_{1}(a)=\tau_{1}\left(a^{\prime}\right)\right)$, then we also have $\left(\alpha(a), v\left(a^{\prime}\right)\right) \in$ $\mathcal{G}_{2}$. Thus there is an isomorphism $\beta: \mathcal{G}_{1} \rightarrow \mathcal{G}_{2}$ such that $\beta\left(\left(a, a^{\prime}\right)\right)=$ $\left(\alpha(a), v\left(a^{\prime}\right)\right)$. This admits a unique extension to a morphism $\beta: M_{1} \rightarrow M_{2}$ through the condition $\beta|N=\alpha| N$.

(b) We shall complete the proof of our lemma by showing that, with $\beta$ defined as above in (a), we have $W_{2}=\beta W_{1}$; for this it will clearly suffice to prove $V_{2}=\beta V_{1}$. Using the notations of 1.1(b), we recall the construction of $V$ as follows (cf. [8, p. 107]; for the notations concerning holomorphic induction of unitary representations of nilpotent groups cf. $[18$, p. 494, f]). (1) For $\mathfrak{h}=\operatorname{pol}(f)$ let us put $\pi_{\mathfrak{h}}=\operatorname{Ind}(\mathfrak{h}, f)$. Since $\pi_{\mathfrak{h}} \sim \pi$ (A.2), there is a unitary map $W: H(\pi) \rightarrow H\left(\pi_{\mathfrak{h}}\right)$ (A.2) such that $W^{-1} \pi_{\mathfrak{h}} W=\pi$. Suppose now that $s$ is fixed in $G_{f}$. There is an $\mathfrak{h}=\operatorname{pol}(f)$ such that $s \mathfrak{h}=\mathfrak{h}$, and we have a canonical construction of a unitary operator $U(\mathfrak{h})$, depending on $s$, acting on $H\left(\pi_{\mathfrak{h}}\right)$ (for the latter also see (c) below). This being so, the crucial fact is that $W^{-1} \cdot U(\mathfrak{h})$ - $W$ is independent of the particular choice of $\mathfrak{h}$ as just specified; we denote it by 
$U(s)$. Summing up, this procedure defines a function from $G_{f}$ with values in the unitary group of $H(\pi)$. (2) For any fixed $b \in \operatorname{Sp}(B)$ there is, with respect to $B$, a maximal self-orthogonal and positive subspace $\mathfrak{m} \subset \mathfrak{b}_{C}$ such that $b \mathfrak{m}=\mathfrak{m}$. Then the number $\arg \left(\operatorname{det}\left(b \mid \mathfrak{b}_{\mathrm{c}} / \mathfrak{m}\right)\right)$ does not depend on the particular choice of $m$ as just described; let us denote it by $\delta(b)$. There is a unique, complex-valued $C^{\infty}$ function $\eta$ on $\mathrm{Mp}(B)$ such that

$$
(\eta(s))^{2} \equiv \delta(\tau(s)) \quad(s \in \operatorname{Mp}(B))
$$

and $\eta(\varepsilon)=-1$. This being so, writing $t$ for the projection $\mathcal{G} \rightarrow \operatorname{Mp}(B)$, we have

$$
V(a) \equiv \eta\left(t\left(a^{-1}\right)\right) U(p(a)) \quad(a \in \mathcal{G})
$$

Returning to the notations of (a) above, by what we have just seen, to complete our proof it is enough to establish that (1) if $s \in G_{1}$ and $s_{2}=\alpha\left(s_{1}\right)$ $\in G_{2}$, then $U_{1}\left(s_{1}\right)=U_{2}\left(s_{2}\right)$; (2) $\eta_{2} \circ v \equiv \eta_{1}$. These points will be dealt with, respectively, in (c) and (d) below.

(c) (1) For some $f \in \mathfrak{n}^{\prime}$ take $\mathfrak{h}=\operatorname{pol}(f)$; then $\pi_{\mathfrak{h}}=\operatorname{Ind}(\mathfrak{h}, f)$ is constructed as follows (cf. [18, proof of Lemma 5.2, p. 498]). Let us set $d=\mathfrak{h} \cap \mathfrak{n}$, $D=\exp (d) \subset N$ (A.4) and let $\chi_{f}$ be the element of $X(D)$ (A.5) satisfying $d x_{f}=i(f \mid d)$. We also write $e=n \cap(\mathfrak{h}+\overline{\mathfrak{h}}), E=\exp (e) \subset N, H=\exp (\mathfrak{h}) \subset$ $N_{\mathrm{C}}$ and observe that $H E$ is open in $E_{\mathrm{C}} \subset N_{\mathrm{C}}$. Let $\chi_{f}$ be a continuous morphism of $H$ into $\mathrm{C}$ such that $d x_{f}=i(f \mid \mathfrak{h})$. Finally, let $d v$ be a positive invariant measure on $D \backslash N$. We now consider the family $\mathcal{H}$ of all measurable functions on $N$ satisfying the following conditions: $(\alpha) g(d x) \equiv$ $x_{f}(d) \cdot g(x)(d \in D, x \in N),(\beta) \int_{D \backslash N}|g(x)|^{2} \cdot d v(p)<+\infty,(\gamma)$ for any fixed $b$ in $N$ the map $h k \mapsto \chi_{f}(h) g(k b)$ is holomorphic on $H E$. This being so, we obtain $H\left(\pi_{\mathfrak{h}}\right)$ by dividing $\mathcal{H C}$ through the variety of elements of vanishing norm, and $\pi_{b}(a)$ arises out of translation on the right by $a \in N$. (2) Assume that $s \in G_{f}$ and that above we have $s \mathfrak{h}=\mathfrak{h}$. Then for an appropriately fixed constant $k>0$, the map $g(x) \mapsto k \cdot g\left(s^{-1} \cdot x s\right)$ defines an isometry of $\mathcal{K}$ onto itself, giving rise to $U(\mathfrak{h})$ on $H\left(\pi_{\mathfrak{h}}\right)$ (cf. (b) above). (3) With notations as in (a) assume that $s_{1} \in G_{1}$ and that $\mathfrak{h}_{1}=\operatorname{pol}\left(f_{1}\right)$ satisfies $s_{1} \mathfrak{h}_{1}=\mathfrak{h}_{1}$. If $s_{2}=$ $\alpha\left(s_{1}\right)$ and $\mathfrak{h}_{2}=\alpha\left(\mathfrak{h}_{1}\right)$ we have $\mathfrak{h}_{2}=\operatorname{pol}\left(f_{2}\right)$ and $s_{2} \mathfrak{h}_{2}=\mathfrak{h}_{2}$. Denoting by $\gamma$ the diffeomorphism $D_{1} \backslash N \rightarrow D_{2} \backslash N$ determined by $\gamma\left(D_{1} x\right)=D_{2} \alpha(x)$, suppose that $v_{2}=\gamma_{*}\left(v_{1}\right)$. Let us write $\pi_{j}=\pi_{\mathfrak{h}}$ if $\mathfrak{h}=\mathfrak{h}_{j}(j=1,2)$, respectively. One easily verifies that the correspondence $g(x) \mapsto g\left({ }^{-1}(x)\right)$ takes $H_{1}$ onto $H_{2}$, giving rise to a unitary map $T: H\left(\pi_{1}\right) \rightarrow H\left(\pi_{2}\right)$ verifying

$$
T\left(\alpha \pi_{1}\right) T^{-1}=\pi_{2} \text { and } T U\left(\mathfrak{h}_{1}\right) T^{-1}=U\left(\mathfrak{h}_{2}\right) \text {. }
$$

From here we prove that $U_{1}\left(s_{1}\right)=U_{2}\left(s_{2}\right)$ (cf. the end of (b) above) as follows. Let $V: H(\pi) \rightarrow H\left(\pi_{1}\right)$ be the unitary map such that $V \pi V^{-1}=\pi_{1}$, and let us put $W=T V$. By what we have just said $W(\alpha \pi) W^{-1}=\pi_{2}$ and, therefore, by 
definition, $U_{2}\left(s_{2}\right)=W^{-1} \cdot U\left(\mathfrak{h}_{2}\right) W$. But the right-hand side is equal to $V^{-1} U\left(\mathfrak{h}_{1}\right) V=U_{1}\left(s_{1}\right)$, completing the proof of our statement.

(d) Let us write $F \equiv \eta_{2} \circ v$; evidently $F$ is a $C^{\infty}$, complex-valued function on $\operatorname{Mp}\left(B_{1}\right)$ such that $F(\varepsilon)=-1$. In this manner, by what we said in (b), it is enough to show that

$$
(F(s))^{2} \equiv \delta_{1}\left(\tau_{1}(s)\right) \quad\left(s \in \mathrm{Mp}\left(B_{1}\right)\right)
$$

Let $s_{1}$ be fixed in $\operatorname{Mp}\left(B_{1}\right)$. If $\mathfrak{m}_{1} \subset\left(\mathfrak{b}_{1}\right)_{\mathrm{C}}$ is maximal self-orthogonal and positive with respect to $B_{1}$, then $m_{2}=w\left(m_{1}\right) \subset\left(b_{2}\right)_{C}$ will be of the same kind in relation to $B_{2}$. If, in addition, we have $\tau_{1}\left(s_{1}\right)\left(\mathfrak{m}_{1}\right)=\mathfrak{m}_{2}$ then, putting $s_{2}=v\left(s_{1}\right)$, we shall have also $\tau_{2}\left(s_{2}\right)\left(\mathfrak{m}_{2}\right)=\mathfrak{m}_{2}$. In this manner we conclude that

$$
\begin{aligned}
\left(F\left(s_{1}\right)\right)^{2} & =\left(\eta_{2}\left(s_{2}\right)\right)^{2}=\delta_{2}\left(\tau_{2}\left(s_{2}\right)\right)=\arg \left(\operatorname{det}\left(\tau_{2}\left(v\left(s_{1}\right)\right) \mid\left(\mathfrak{b}_{2}\right)_{\mathrm{C}} / \mathfrak{m}_{2}\right)\right) \\
& =\arg \left(\operatorname{det}\left(s_{1} \mid\left(\mathfrak{b}_{1}\right)_{\mathrm{C}} / \mathfrak{m}_{1}\right)\right)=\delta_{1}\left(s_{1}\right),
\end{aligned}
$$

completing the proof of Lemma 1. Q.E.D.

2.1. Let $G$ be a connected Lie group with the Lie algebra $g$, and $g$ a fixed element of $g^{\prime}$. We write $f=g \mid \mathfrak{n}$, assume $f \neq 0$ and consider, as in 1.1(a), the group $\mathcal{G}$. It acts, via $p(\mathcal{G})=G_{f}$, on $g^{\prime}$ and thus, forming $\mathcal{G}_{g}$ (A.l), we can observe that $\left(\left(\mathcal{G}_{g}\right)_{0}, p\right)$ provides a covering, of a degree not exceeding two, of $\left(G_{g}\right)_{0}$ (A.6). With these notations we introduce the following

DEFINITION. We call the element $g \in g^{\prime}$ admissible if and only if there is a $\chi_{g} \in X\left(\left(\mathcal{G}_{g}\right)_{0}\right)$ (A.5) such that $d \chi_{g}=i\left(g \mid g_{g}\right)$ and, if $\varepsilon \in\left(\mathcal{G}_{g}\right)_{0}$ then $\chi_{g}(\varepsilon)=$ -1 .

We denote by $W(G)$ the set of all admissible elements of $g^{\prime}$. One easily verifies (cf. (a), proof of Lemma 1) that $W(G)$ is invariant under $\operatorname{Ad}(G)$.

To clarify the content of the above definition, we consider two special cases.

(1) Assume first that $G$ is compact. We start by recalling, that in this case $G_{g}$ is always connected. In fact, since $g=[\mathfrak{g}, q]+g^{\natural}$ we have $G=[G, G] \cdot G^{\natural}$ (A.7) and thus we can suppose that $g$ is semisimple. We identify $g$ to $g^{\prime}$ by aid of the Killing form. If $a \in G$ and $X \in \mathrm{g}$, we have $a X=X$ if and only if $a$ commutes with the closure $T$ of $\{\exp (t X) ; t \in \mathrm{R}\}$. Hence the desired conclusion follows from the existence of a maximal torus containing both $a$ and $T$ (cf. e.g. $\left[22,3.9 .4\right.$, p. 72]). Since $g^{\natural}=n$, we have $n_{f}=n$, and thus $\left(\left(G_{g}\right)_{0}, p\right)$ is a simple covering of $G_{g}$. Summing up, if $G$ is compact, $W(G)$ is composed of all those elements $g$ of $\mathrm{g}^{\prime}$ for which there is a $\chi_{g} \in X\left(G_{g}\right)$ satisfying $d x_{g}=i\left(g \mid g_{g}\right)$.

(2) Suppose next that $G$ is a simply connected solvable Lie group. We claim that then $W(G)=g^{\prime}$. To show this let us recall that any connected subgroup of $G$ is closed and simply connected. This implies first that $\left(\left(\mathcal{G}_{g}\right)_{0}, p\right)$ is a simple covering of $\left(G_{g}\right)_{0}$, and next, by the same token, that $\chi_{8} \in X\left(\left(\mathcal{G}_{g}\right)_{0}\right)$, as 
required in the definition, exists for all $g \in g^{\prime}$.

2.2. The following lemma will often be used in the sequel. Its demonstration is close to that of [18, Lemma 6.1, p. 500].

LEMMA 2. Let $G$ be a connected Lie group with the Lie algebra $\mathfrak{g}, \mathfrak{m}$ an ideal of $\mathfrak{g}$ and $M$ the corresponding connected analytic subgroup. For some fixed $g \in g^{\prime}$ we put $h=g \mid \mathfrak{m}$ and $g_{\lambda}=\mathfrak{m}+g_{h}$. Then we have $\left(M_{h}\right)_{0} g=g+g_{\lambda}^{1}$.

Proof. (a) We start by observing that if $n$ is an integer not smaller than two and $\left\{l_{j} ; 1<j<n\right\} \subset \mathfrak{m}_{h}$, then $l_{1} \cdot l_{2} \cdots l_{n} g=0$. In fact, writing $v$ for the left-hand side, we have for an arbitrary $l \in g:(l, v)=\left(\left[l_{n}, k\right], g\right)$, where $k \in \mathfrak{m}$. Hence, to obtain the desired conclusion it is enough to note that $l \in \mathfrak{g}$ belongs to $g_{h}$ if and only if $([u, l], g)=0$ for all $u$ in $\mathrm{m}$. We conclude from this at once that $\left(M_{h}\right)_{0} g=g+\mathfrak{m}_{h} \cdot g$. Since $\mathfrak{m}_{h} g$ is clearly orthogonal to $\mathfrak{m}+\mathfrak{g}_{h}=g_{\lambda}$ we infer that also $\left(M_{h}\right)_{0} g \subseteq g+g_{\lambda}^{1}$.

(b) By the preceding, to complete our proof it suffices to show that $\operatorname{dim}\left(g_{\lambda}^{+}\right)=\operatorname{dim}\left(\mathfrak{m}_{h} \cdot g\right)$. But here the right-hand side is equal to $\operatorname{dim}\left(\mathfrak{m}_{h}\right)-$ $\operatorname{dim}\left(\mathfrak{m}_{\mathfrak{g}}\right)$, while on the left we have $\operatorname{dim}(\mathfrak{g})-\operatorname{dim}\left(\mathfrak{g}_{\lambda}\right)$. Since $\operatorname{dim}\left(\mathfrak{g}_{\lambda}\right)=\operatorname{dim}(\mathfrak{m})$ $+\operatorname{dim}\left(\mathfrak{g}_{h}\right)-\operatorname{dim}\left(\mathfrak{m}_{h}\right)$, it is enough to remark that $\operatorname{dim}\left(\mathfrak{g}_{h}\right)=\operatorname{dim}(g)-$ $\operatorname{dim}(\mathfrak{m})+\operatorname{dim}\left(\mathfrak{m}_{\mathfrak{g}}\right)$. Q.E.D.

2.3. In this subsection $\mathfrak{d}$ will denote a Lie algebra, the radical of which is nilpotent and, at the same time, cocompact. The principal result here is Proposition 1 which, because of its intrinsic interest, provides somewhat more than needed in the sequel (for this Lemma 6 would suffice). Let us put $L=\exp (\delta)$ (A.4). If $0 \neq f \in \mathfrak{n}^{\prime}$, we can form, as in 1.1(d), but now with respect to $L$, the diagram

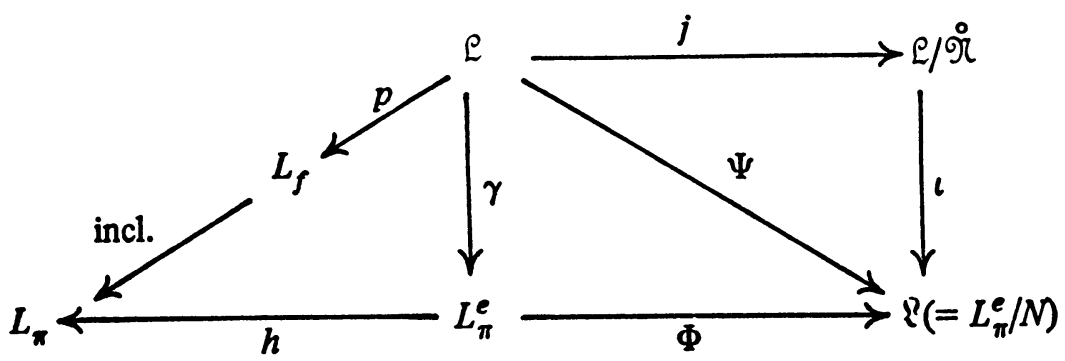

Observe that by virtue of our assumption $\mathfrak{R}$ is compact. In fact, it is an extension, by the circle group $\Psi(\Re)=\mathfrak{T}\left(\subset \mathbb{R}^{\natural}\right)$, of $\mathcal{L} / \Re$; but the latter is isomorphic to $L_{f} / N_{f}=L_{\pi} / N$, which is compact since $L / N$ is. We set $\mathfrak{\Omega}_{0}=3=\exp (3)($ A.4, A.6).

LEMMA 3. With the previous notations and assumptions suppose that $h \in \mathfrak{d}^{\prime}$ is 
such that $h \mid \mathfrak{n}=f \neq 0$. Let us write $h \mid \delta_{f}=\delta \Psi(\kappa)\left(\kappa \in \jmath^{\prime}\right)$. Then $h$ belongs to $W(L)$ if and only if $\kappa$ belongs to $W(3)$.

Proof. A. We assume first that $h \in W(L)$. We recall that by the Definition of 2.1 this is equivalent to the existence of $\chi_{h} \in X\left(\left(\mathcal{L}_{h}\right)_{0}\right)$ such that $d \chi_{h}=i\left(h \mid \delta_{h}\right)$, and if $\varepsilon \in\left(\mathcal{E}_{h}\right)_{0}$ then also $\chi_{h}(\varepsilon)=-1$. By what we said in the first example loc. cit., to prove that then $\kappa \in W(3)$ we have to exhibit a character $\chi_{\kappa} \in X\left(Z_{\kappa}\right)$ satisfying $d \chi_{\kappa}=i\left(\kappa \mid z_{\kappa}\right)$.

(a) Setting ${ }^{-1} \Psi\left(3_{k}\right)=\Re \supset \Re$ and $p(\Re)=M=\exp (\mathfrak{m})$ (A.4) we claim that $\Re=\left(\mathscr{L}_{h}\right)_{0} \cdot \Re$. In fact, to this end it is enough to show that $M=\left(L_{h}\right)_{0}$ - $N_{f}$. But (1) by $h \mid \delta_{f}=\delta \Psi(\kappa)$, we have

$$
p\left(\stackrel{-1}{\Psi}^{-1}\left(\mathfrak{I}_{\kappa}\right)\right)=\left\{a ; a \in L_{f}, a h\left|\delta_{f}=h\right| \delta_{f}\right\} .
$$

Let us write $\delta_{\pi}=\mathfrak{n}+\delta_{f}$. If $a$ belongs to the previous set we obviously have $a h\left|\delta_{\pi}=h\right| \delta_{\pi}$. Hence, by Lemma 2 there is an $n \in N_{f}$ such that $a h=n h$ implying $a \in L_{h} \cdot N_{f}$. Conversely, we can infer from this relation that $a h \in h$ $+\delta_{\pi}^{\perp}$ and thus $a h\left|\delta_{f}=h\right| \delta_{f}$. Summing up, we have just proved that

$$
p\left(\Psi^{-1}\left(\mathfrak{R}_{\kappa}\right)\right)=L_{h} \cdot N_{f}
$$

Observe that this implies $\mathfrak{m}=\mathfrak{d}_{h}+\mathfrak{n}_{f}$. (2) Both now and later we repeatedly use the following statement, the verification of which we leave to the reader. Suppose that $\mathbb{R}$ is a closed subgroup of $\mathfrak{R}$ containing $\mathfrak{I}=\Psi(\mathscr{T})$. Then we have $p(\Psi(\Omega))_{0}$. Since $3_{\kappa}=\left(\mathfrak{R}_{\kappa}\right)_{0}$, using (1) we conclude by aid of the quoted statement that

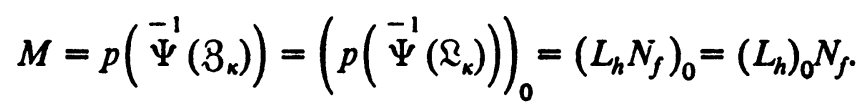

(b) We want to show next that there is a $\chi \in X(\mathscr{R})$ such that $\chi \mid\left(\varrho_{h}\right)_{0}=\chi_{h}$ and $\chi \mid \Re=\varphi_{f}$ (for the latter cf. 1.1(b)). To this end it suffices to prove that the restrictions of $\chi_{h}$ and of $\varphi_{f}$, respectively, to $\left(\mathscr{L}_{h}\right)_{0} \cap \Re$ coincide. But we clearly have $\left(\mathfrak{L}_{h}\right)_{0} \cap \mathscr{T}_{0}=\exp \left(\mathfrak{n}_{h}\right) \subset \mathfrak{L}$ (A.4) and, therefore, if the former subgroup is larger it is equal to $\exp \left(\mathfrak{n}_{h}\right) \cup \varepsilon \cdot \exp \left(\mathfrak{n}_{h}\right)$. In this manner the desired conclusion follows from $d \chi_{h}\left|\mathfrak{n}_{h}=i\left(f \mid \mathfrak{n}_{h}\right)=d \varphi_{f}\right| \mathfrak{n}_{h}$, and that if $\varepsilon \in$ $\left(\mathcal{L}_{h}\right)_{0}$ then $\chi_{h}(\varepsilon)=-1=\varphi_{f}(\varepsilon)$. Observe that $d \chi=i(h \mid \mathrm{m})$.

Since $\operatorname{ker}(\chi) \subseteq \mathscr{\Upsilon}^{\circ}=\operatorname{ker}(\Psi)$, there is an $\omega \in X\left(3_{k}\right)$ such that $\omega \circ(\Psi \mid \Re)$ $=x$. To complete our proof that $\kappa \in W(ß)$, it is enough to show that $d \omega=i\left(\kappa \mid z_{k}\right)$. If $z_{k} \ni v=d \Psi(u)(u \in \mathfrak{m})$, we have $\omega(\exp (v))=\chi(\exp (u))=$ $\exp [i(u, h)]$ which, by $h \mid \delta_{f}=\delta \Psi(\kappa)$, coincides with $\exp [i(v, \kappa)]$.

B. Let us assume now that $\kappa \in W(3)$. Putting $\chi=\chi_{k} \circ(\Psi \mid \Re) \in X(\Re)$, we again have $d \chi=i(h \mid \mathrm{m})$. Observe that $\chi \mid \Re=\varphi_{f}$. In fact, since $d\left(\chi \mid \Re_{0}\right)=$ 
$i\left(f \mid \mathfrak{n}_{f}\right)=d\left(\varphi_{f} \mid \Re_{0}\right)$, the desired conclusion follows from $\operatorname{ker}(\chi) \subseteq \dot{\vartheta}$. Hence, in particular, $\chi(\varepsilon)=\varphi_{f}(\varepsilon)=-1$. Writing $\psi=\chi \mid\left(\varrho_{h}\right)_{0}$, we therefore have, $d \psi=i\left(h \mid \delta_{f}\right)$ and also, if $\varepsilon \in\left(\mathscr{E}_{h}\right)_{0}, \psi(\varepsilon)=-1$, completing the proof that $h \in W(L)$, since $\psi$ satisfies the conditions placed on $\chi_{h}$ by the Definition of 2.1. Q.E.D.

The following lemma is inspired by [11]; cf., in particular, pp. 205-206, loc. cit. The map $\delta$ introduced here can be shown to be the "classical" one; for instance, the character of $\rho \in \hat{G}$ admits a simple integral expression by aid of a translate of $\delta(\rho)$ (for the semisimple case cf. e.g. [15, Proposition 1, p. 259]). This is what we intend to express by the adjective "canonical".

Lemma 4. Assume that $G$ is a compact, connected Lie group. There exists a canonical bijection $\delta$, equivariant with respect to $\operatorname{Aut}(G)$, from the dual of $G$ onto $W(G) / G$.

Proof. (a) Let $g$ be the Lie algebra of $G$. We set $z=g^{\natural}$ (A.7) and $\mathfrak{g}_{1}=[\mathfrak{g}, \mathfrak{g}]$. The latter is a compact Lie algebra and $\mathfrak{g}$ is equal to the direct sum $g_{1}+z$. We select a maximal abelian subalgebra $a$ of $g$ to be kept fixed until further notice, and write $a_{1}=a \cap g_{1}$. Let us put $\mathfrak{h}=a_{c}$ and $\mathfrak{h}_{1}=\left(a_{1}\right)_{c}$. We fix a Weyl chamber $P$ in $i a_{1} \subset \mathfrak{h}_{1}$ and denote by $\mathscr{D}^{\prime}\left(\subset \mathfrak{h}_{1}^{\prime}\right)$ the set of the dominant integral functions corresponding to $P$. Let us write

$$
\Gamma_{G}=\{l ; l \in \mathfrak{a}, \exp (l)=\text { unity }\},
$$

and

$$
\mathscr{D}=\left\{\Lambda ; \Lambda \in \mathfrak{h}^{\prime}, \Lambda\left(\Gamma_{G}\right) \subseteq 2 \pi i Z, \Lambda \mid \mathfrak{h}_{1} \in \mathscr{D}^{\prime}\right\} .
$$

This being so, we recall that there exists a canonical bijection between $\hat{G}$ and $\mathscr{D}$ (cf. [22, 4.6.12, p. 98]). Let us denote by $g_{1}^{\prime}$ and $z^{\prime}$ the annihilator with respect to the canonical bilinear form on $g \times g^{\prime}$ of $z$ and $g_{1}$ respectively; we then have the direct sum representation $g^{\prime}=g_{1}^{\prime}+z^{\prime}$. We shall identify $\mathfrak{g}^{\prime}$ to $g$ by aid of the Killing form on the latter. Now let $\rho$ be an element of $\hat{G}$ and $\Lambda$ the corresponding linear form in $\mathscr{D}$. We define $g \in \mathfrak{g}^{\prime}$ by the condition that $\Lambda(z) \equiv i(z, g)(z \in z)$ and $g \mid \mathfrak{g}_{1}=\lambda$, where $\lambda \in a_{1}$ is determined by $\Lambda(a) \equiv$ $i B(a, \lambda)\left(a \in a_{1}\right)$. Observe that in this fashion $\Lambda(a) \equiv i(a, g)(a \in \mathfrak{a})$. We next show that $g \in g^{\prime}$, as just defined, belongs to $W(G)$. To this end let us first put $\exp (a)=T$ (A.4); $T$ is a maximal torus in $G$. Next we remark that, by $\Lambda \in \mathscr{D}$, given $\gamma \in \Gamma_{G},(\gamma, g)$ is of the form $2 \pi m$, where $m$ is an integer. From this we conclude that there is a $\chi \in X(T)$ (A.5) such that $d \chi=i(g \mid \mathrm{a})$ or $g \mid \mathfrak{a} \in W(T)$. We claim this implies $g \in W(G)$. Since $G_{g}$ is connected, to prove this we can evidently assume $G_{g}=G$; observe that this is equivalent to $g \mid g_{1}=0$. Let us write $G_{1}=\exp \left(g_{1}\right)(=[G, G]), Z=\exp (z)\left(=\left(G^{\natural}\right)_{0}\right)$ and $T_{1}=\exp \left(a_{1}\right)$. Since $T=T_{1} \cdot Z$, and $T_{1}$ is a maximal torus in $G_{1}$, we have $G_{1} \cap T=T_{1}\left(G_{1} \cap Z\right)=T_{1}$, implying that $\chi \mid\left(G_{1} \cap T\right) \equiv 1$. Since $G=G_{1} \cdot$ 
$T$, there is a $\varphi \in X(G)$ such that $\varphi|T \equiv \chi, \varphi| G_{1} \equiv 1$, whence clearly $d \chi=i g$, proving that $g \in W(G)$. Summing up, given $\rho \in \hat{G}$, the above procedure assigns to it a well-determined element $g \in W(G)$. We define $\delta: \hat{G} \rightarrow$ $W(G) / G$ by $\delta(\rho)=G g$. (b) Let us assume next that the elements $\rho_{j}(j=1$, 2) of $\hat{G}$ are such that $\delta\left(\rho_{1}\right)=\delta\left(\rho_{2}\right)$; we claim that then $\rho_{1}=\rho_{2}$. We shall distinguish the objects, introduced as in (a) above, relative to $\rho_{j}$ by the index $j=1,2$; we have to show that $\Lambda_{1}=\Lambda_{2}$. Our assumption implies that there is an $a \in G$ such that $\lambda_{2}=a \lambda_{1}$. We claim that one can assume that at the same time $a \mathfrak{a}=\mathfrak{a}$. In fact, let us put $h=\lambda_{2}$ and $\mathfrak{b}=a \mathfrak{a}$. Since $\mathfrak{a}$ and $\mathfrak{b}$ are maximal abelian in $g_{h}$ (A.1), there is an $s \in G_{h}$ such that $a=s \mathfrak{b}$ (cf. e.g. [22, 3.6.11, (4), p. 63]). Setting $k=s a$ we then have $k \lambda_{1}=\lambda_{2}(=h)$ and $k a=a$. In this manner we can assume that $a \Lambda_{2}=\Lambda_{1}$. Let us write $\Lambda_{j}^{\prime}=\Lambda_{j} \mid \mathfrak{h}_{1}(j=1,2)$. Then $\Lambda_{1}^{\prime}$ and $\Lambda_{2}^{\prime}$ differ by an action of the Weyl group and thus, being dominant integral, $\Lambda_{1}^{\prime}=\Lambda_{2}^{\prime}$. But by $\mathfrak{h}=\mathfrak{h}_{1}+z_{c}$ this implies $\Lambda_{1}=\Lambda_{2}$ and $\rho_{1}=\rho_{2}$. Summing up, by what we have just seen, $\delta$ is an injection $\hat{G} \rightarrow$ $W(G) / G$. (c) We prove next that $\delta$ is surjective. Let $O \in W(G) / G$ be given. We can choose $g \in O$ such that $g_{g} \supseteq \mathfrak{a}$ and that $i(g \mid \mathfrak{a}) \in \bar{P}$ (cf. (a)). Then defining $\Lambda \in \mathfrak{h}^{\prime}$ by $\Lambda(a) \equiv i(a, g)(a \in \mathfrak{a})$, it suffices to show that $\Lambda \in \mathscr{D}$. By $g \in W(G)$ we have $g \mid a \in W(T)$ and thus clearly $(\gamma, g) \in 2 \pi \cdot Z\left(\gamma \in \Gamma_{G}\right)$ implying that $\Lambda\left(\Gamma_{G}\right) \subset 2 \pi i \cdot Z$. Denoting by $\alpha_{1}, \alpha_{2}, \ldots, \alpha_{r}$ the system of simple roots corresponding to $P$ we set $H_{j}=H_{\alpha} / \alpha\left(H_{\alpha}\right)$ if $\alpha=\alpha_{j}(1<j<$ $r)$. To conclude our proof it is enough to establish that $\Lambda \mid h_{1} \in \mathcal{D}^{\prime}$ or that $\Lambda\left(H_{j}\right)$ is a nonnegative integer. Since $i\left(g \mid a_{1}\right) \in \bar{P}$, we have $\Lambda\left(H_{j}\right)>0$. Hence to arrive at the desired conclusion it suffices to recall that $2 \pi i\left(\mathrm{ZH}_{1}+\mathrm{ZH}_{2}\right.$ $\left.+\cdots+Z H_{r}\right) \subset \Gamma_{G}$ (cf. [22, 4.6.7, p. 96]). Thus $\delta$ is a bijection $\hat{G} \rightarrow$ $W(G) / G$. (d) To complete the proof of our lemma, let us first note that the map $\delta$ is independent of the particular choice of the maximal abelian subalgebra $\mathfrak{a}$ in $\mathfrak{g}$. In fact, let $\mathfrak{b}$ be a second one of this kind; we can assume that $\mathfrak{b}=s \mathfrak{a}$ $\left(s \in G\right.$ ). If, for a given $\rho \in \hat{G}, \Lambda$ and $\Lambda^{\prime}$ are the corresponding linear forms, as in (a), on $a_{C}$ and $\mathfrak{b}_{C}$, respectively, we have $\Lambda^{\prime}=s \Lambda$. Let $g$ and $g^{\prime}$ be the elements of $g^{\prime}$ obtained, starting from, respectively, $\Lambda$ and $\Lambda^{\prime}$, as in (a), via, respectively, $a$ and $\mathfrak{b}$. We finish the proof of our assertion by showing that $\boldsymbol{g}^{\prime}=s \boldsymbol{g}$. But, by construction, for all $l$ in $\mathfrak{b}$ :

$$
i\left(l, g^{\prime}\right) \equiv \Lambda^{\prime}(l) \equiv \Lambda\left(s^{-1} l\right) \equiv i\left(s^{-1} l, g\right) \equiv i(l, s g)
$$

or $\left(l, g^{\prime}\right) \equiv(l, s g)(l \in \mathfrak{b})$, whence the desired conclusion follows by recalling that $g^{\prime}\left|\mathfrak{g}_{1}, s g\right| \mathfrak{g}_{1} \in \mathfrak{b}_{1} \quad\left(\mathfrak{b}_{1}=\mathfrak{b} \cap \mathfrak{g}_{1}\right)$. (e) Let us prove finally that $\delta$ is equivariant with respect to $\operatorname{Aut}(G)$. If $\alpha \in \operatorname{Aut}(G)$, a maximal abelian in $g$, and if $g \in \delta(\rho)$ corresponds to it as in (a), the reasoning of (d) shows that $\alpha g \in \delta(\alpha \rho)$, and thus

$$
\delta(\alpha \rho)=G \alpha g=\alpha(G g)=\alpha(\delta(\rho)),
$$


completing the proof of Lemma 4. Q.E.D.

The following lemma provides an analogue for the statements summarized in 1.1(b) assuming that $N$, loc. cit., is replaced by some connected, invariant compact subgroup. Since here we do not need the analogue of Lemma 1, we can formulate our statement somewhat more simply.

In the following, $\mathfrak{R}$ will denote a locally compact group, $3=\exp (z)(\mathrm{A} .4) \mathrm{a}$ fixed connected, invariant and compact Lie subgroup of $\mathfrak{R}$. For a nontrivial $\sigma$ in $\hat{\jmath}$ we fix a $\kappa \in \delta(\sigma) \subset W(3)$ (cf. Lemma 4).

LEMMA 5. With the previous notations there is a continuous unitary representation $V$ of $\mathfrak{M}_{\kappa}$ on $H(\sigma)$ (A.1, A.2) such that

$$
\begin{aligned}
V(a) \sigma(z) V\left(a^{-1}\right) & =\sigma\left(a z a^{-1}\right) & & \left(z \in B_{3} a \in \mathbb{M}_{\kappa}\right), \\
V(z) & \equiv \overline{\chi_{\kappa}(z)} \sigma(z) & & \left(z \in B_{\kappa}\right) .
\end{aligned}
$$

Proof. This is a simple implication of the Borel-Weil theorem. The following brief summary of its relevant part is organized in analogy with the nilpotent theory (cf. the proof of Lemma 1, in particular (c), loc. cit.); for more details, cf. [22, Chapter 6, in particular, 6.3 (p. 153)]. We now write $\mathfrak{g}=[\mathfrak{z}, z]$ and identify $\mathfrak{g}^{\prime}$ to $\mathfrak{g}$ by aid of the Killing form. Let $\mathfrak{a} \subset \mathfrak{g}_{\boldsymbol{k}}$ be a maximal abelian subalgebra and, setting $\mathfrak{h}_{1}=a_{C}$ and $\lambda=\kappa \mid \mathfrak{a}(\lambda \in \mathfrak{a})$, we select a Weyl chamber $P \subset i \mathfrak{a} \subset \mathfrak{h}_{1}$ such that $i \lambda \in \bar{P}$. We denote by $\Delta_{+}\left(\Delta_{-}\right)$ the corresponding set of positive (resp. negative) roots and put $\Delta=\Delta_{+} \cup \Delta_{-}$; $g_{\alpha} \subset g_{C}$ stands for the root space of $\alpha \in \Delta$. Setting $\mathscr{F}_{0}=\{\alpha ; \alpha \in \Delta, \alpha(\lambda)=$ $0\}$, we have, evidently, $\left(\mathfrak{g}_{\lambda}\right)_{\mathrm{C}}=\mathfrak{h}_{1}+\Sigma_{\alpha \in \mathfrak{J}_{0}} \mathfrak{g}_{\alpha}$. Let us observe next that if $\mathscr{F}_{+}=\{\alpha ; \alpha \in \Delta, \alpha(\lambda)>0\}$, we have $\mathscr{F}_{+} \subset \Delta_{+}$. In fact, if $\alpha \in \Delta_{-}$then, by $i \lambda \in \bar{P}, \alpha(i \lambda)<0$. Thus $\mathfrak{m}=\Sigma_{\alpha \in \mathscr{F}_{+}} g_{\alpha}$ is a nilpotent subalgebra of $z c$. Furthermore, if $\alpha \in \mathscr{F}_{0}$ and $\beta \in \mathscr{F}_{+}$, we have clearly $\alpha+\beta \in \mathscr{F}_{+}$; therefore $\left[\mathfrak{m},\left(\mathfrak{g}_{\lambda}\right)_{\mathfrak{c}}\right] \subseteq \mathfrak{m}$ and $\mathfrak{h}_{2}=\left(\mathfrak{g}_{\lambda}\right)+\mathfrak{m}$ is a subalgebra. Let us set $\mathfrak{h}=\mathfrak{h}_{2}+\left(\mathfrak{g}^{\mathfrak{h}}\right)_{\mathfrak{c}}$ and write $H=\exp (\mathfrak{h}) \subset Z_{\mathrm{C}}$ (A.4). There is a holomorphic character, to be denoted again by $\chi_{k}$, of $H$ satisfying $d \chi_{\kappa}=i(\kappa \mid \mathfrak{h})$. Also, one can show that $H \cap B=3_{\kappa}$. Therefore if $f$ is a complex-valued function on 3 satisfying, for any $z \in Z_{\kappa}$ and $x \in Z, f(z x)=\chi_{k}(z) f(x)$, there is a well-defined function $F$ on the open subset $H 3$ of $3_{\mathrm{c}}$, such that $F^{\prime}(h x)=\chi_{x}(h) f(x)(h \in H, x \in Z)$. This being said, let us denote by $\mathcal{H C}$ the linear space of all those functions $f$ as above, for which $F$, as just defined, is holomorphic on $H 3$. Then $\operatorname{dim}(\mathcal{H C})<$ $+\infty$, and the representation of 3 , corresponding to translations on the right of elements of $\mathcal{T}$, is equivalent to $\sigma$. From here we prove our lemma as follows. Suppose that $\tau \in \operatorname{Aut}(3)$ is such that $\tau \kappa=\kappa$. We claim that this implies $\tau(\mathfrak{h})=\mathfrak{h}$. To this end it evidently suffices to show that $\tau\left(\mathfrak{h}_{2}\right)=\mathfrak{h}_{2}$. Our assumption at once yields $\tau \lambda=\lambda$, and thus we can write

$$
\tau\left(\mathfrak{h}_{2}\right)=\left(\mathfrak{g}_{\lambda}\right)_{\mathrm{C}}+\sum_{\alpha \in \mathscr{F}^{\prime}} \mathfrak{g}_{\alpha}
$$


where $\mathscr{F}^{\prime} \cap \mathscr{F}_{0}=0$. Therefore it is enough to prove $\mathscr{F}^{\prime} \subseteq \mathscr{F}_{+}$. If $B$ is the Killing form and $h \in \mathfrak{h}_{2}$, then $i B([h, \bar{h}], \lambda)>0$; but then the same is also valid for $h \in \tau\left(\mathfrak{h}_{2}\right)$. Since clearly $\tau\left(\mathcal{B}_{\kappa}\right)=3_{\kappa}$ and $\tau \chi_{\kappa}=\chi_{\kappa}$ (A.3), we conclude that the map $f(x) \mapsto f(\tau(x))$ transforms $\mathcal{X}$ into itself. Let $d v$ be an invariant measure on $3_{x} \backslash 8$, then the said substitution leaves the unitary metric, defined on $\mathcal{X}$ by $\int_{3 \times 13}|f(x)|^{2} \cdot d v$, invariant. In this manner, to obtain the unitary representation $V$, appearing in the statement of our lemma, of $\mathfrak{M}_{x}$ on $H(\sigma)$, it suffices to define for $a \in \mathbb{M}_{\kappa}, f \in \mathcal{H}:(V(a) f)(x) \equiv f\left(a^{-1} x a\right)(x \in$ 3). Q.E.D.

REMARK 1. Given a nontrivial unitary representation (as above) of 3 and a fixed $\kappa \in \delta(\sigma) \subset W(3)$ (A.2), we construct a diagram, analogous to that of 1.1(d), in the following manner. First, replacing $G, N$ and $\pi$, loc. cit., through, respectively $\mathfrak{M}, 3$ and $\sigma$ (all as above), we form $\mathfrak{M R}_{\sigma}$. Next, as in 1.1(c)(5), we observe that 3 can be canonically identified to an invariant subgroup (to be denoted again by 3 ) of $\mathfrak{M}_{\sigma}^{e}$. We set $\Gamma=\mathfrak{D}_{\mathfrak{c}}^{e} / 3$ and write $\varphi$ for the corresponding canonical homomorphism. We can now define, as in 1.1(d), an injective homomorphism $\varepsilon: \mathfrak{M}_{\kappa} \rightarrow \mathfrak{M}_{\sigma}^{e}$ through the following remarks. Let $\sigma^{\prime}$ be a projective extension, giving rise to the 3 invariant cocycle $\alpha$, of $\sigma$ to $\mathfrak{R}_{e}$, and let us form, similarly as in 1.1(c)(5), the group $\&\left(\sigma^{\prime}\right)$. Next we observe that if $V$ is as in Lemma 5 , there is a unique measurable map $k$ of $\mathbb{M}_{k}$ into the circle group such that $V(a) \equiv k(a) \sigma^{\prime}(a)\left(a \in \mathbb{M}_{k}\right)$. This first implies, as loc. cit., that $\alpha \mid\left(\mathfrak{M}_{\kappa} \times \mathfrak{M}_{\kappa}\right)=\delta k$, and thus we can define an injective homomorphism $\eta: \mathfrak{R}_{\kappa} \rightarrow \mathbb{B}\left(\sigma^{\prime}\right)$ by $\eta(a)=(a, k(a))$, whence the existence of $\varepsilon$ as above again follows as loc. cit. Let us put $\dot{3}_{k}=\operatorname{ker}\left(\chi_{k}\right)$; since $\kappa \neq 0$ this is a proper subgroup of $3_{k}$. Using $V(z) \equiv \overline{\chi_{k}(z)} \sigma(z)\left(z \in B_{k}\right)$ we next observe that $k(z) \equiv \overline{\chi_{k}(z)}$ and therefore $\operatorname{ker}(\varphi \circ \varepsilon)=\dot{3}_{k}$. Let us also remark that by Lemma 4 we have $\mathfrak{R}_{\sigma}=\mathfrak{R}_{\kappa} \cdot 3$. In fact, since $\delta(\sigma)=Z_{\kappa}$, we conclude that if $a \in \mathfrak{R}: \delta(a \sigma)=a \delta(\sigma)=z 3_{\kappa} . \delta$ being injective, we have $a \sigma=\sigma$ if and only if $\delta(a \sigma)=\delta(\sigma) \circ \tau$ if $a 3 \kappa=3 \kappa$, which is equivalent to $a \in \mathbb{M}_{\kappa} \cdot 3$. This immediately implies $\mathfrak{M}_{\sigma}^{e}=\varepsilon\left(\mathfrak{R}_{\kappa}\right) \cdot 3$ and $\varphi\left(\varepsilon\left(3_{\kappa}\right)\right)=\Gamma$. In this manner we obtain the following diagram:

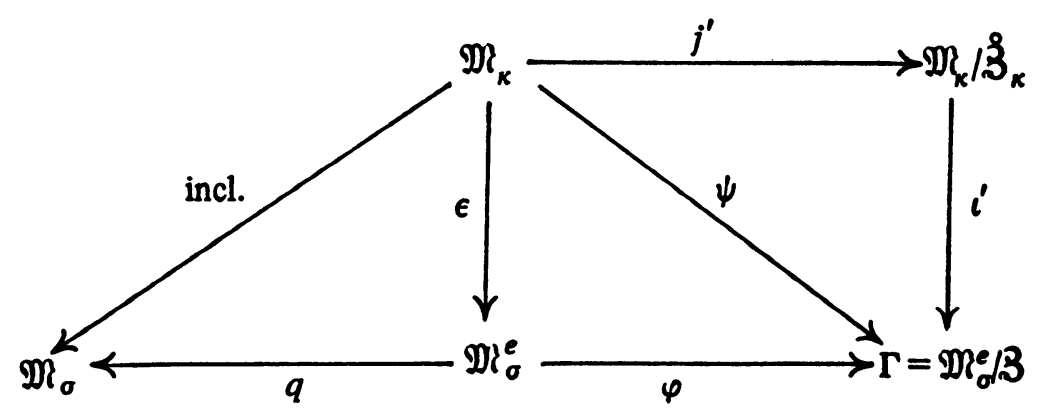


where $\psi$ is a surjective homomorphism, and the unexplained symbols are to be interpreted analogously to the diagram of 1.1(d).

ReMARK 2. From the preceding we can derive the following observation which is similar to that of $1.1(\mathrm{e})$. Let us suppose that the group $\mathfrak{R}$ contains $\mathfrak{M}$ and 3 (as before) as closed invariant subgroups. If $n \in \mathfrak{R}$ is such that $n \kappa=\kappa$, it induces an automorphism $\zeta^{\prime}\left(\eta^{\prime}\right)$ of $\mathfrak{D}_{\kappa}$ (resp. $\left.\mathfrak{M} \mathcal{F}_{\sigma}\right)$ such that $\zeta^{\prime}\left(\hat{\zeta}_{k}\right)=\dot{\zeta}_{\kappa}$ (resp. $\eta^{\prime}(\xi)=3$ ). Hence there are automorphisms $\zeta$ and $\eta$ of $\Gamma$ satisfying $\zeta \circ \psi=\zeta^{\prime}, \eta \circ \varphi=\eta^{\prime}$. This being said, we have $\eta=\zeta$.

We recall that now $\delta$ denotes a Lie algebra, the radical of which is nilpotent and cocompact. If $\mathfrak{n}$ is nilpotent and $N=\exp (\mathfrak{n})$ (A.4), there is a canonical bijection $\zeta$, to be quoted as the Kirillov isomorphism, from $\hat{N}$ onto $\mathfrak{n}^{\prime} / N$, which is also $\operatorname{Aut}(N)$ equivariant (for this cf. e.g. [18, Remark 4.1, p. 497]). For the explanation of the adjective "canonical" in the next lemma cf. Remark 3 below.

Leman 6. Let us put $L=\exp (\delta)$. There is a canonical $\operatorname{Aut}(L)$ equivariant surjection $\eta$ from $\hat{L}$ onto $W(L) / L$ such that any $\eta$ orbit in $\hat{L}$ is finite. If $\delta$ is nilpotent, $\eta$ coincides with the Kirillov isomorphism.

Proof. We continue to denote the nilradical of $\delta$ by $\mathfrak{n}$ and $\operatorname{set} N=\exp (\mathfrak{n})$ $C L$.

(a) We write $F=\{\lambda ; \lambda \in \hat{L}, \operatorname{ker}(\lambda) \supset N\} ; F$ is clearly invariant under $\operatorname{Aut}(L)$. Let us put $g=\delta / \mathfrak{n}$; by assumption $g$ is compact semisimple. Hence $G=\exp (g)$ (A.4) is compact. There is a canonical identification between $g^{\prime}$ and $\mathfrak{n}^{\perp} \subset \mathfrak{d}^{\prime}$ and, as one verifies easily, $W(G)=W(L) \cap \mathfrak{n}^{\perp}$. We denote by $i$ the canonical homomorphism $L \rightarrow G$. For $\lambda \in F$ let us write $\lambda=\sigma \circ i$; the map $\sigma \mapsto \lambda$ is a bijection between $\hat{G}$ and $F \subset \hat{L}$. With these notations and identifications let us define $\eta(\lambda)=\delta(\sigma) \subset W(L) \cap \mathfrak{n}^{\perp}$ (cf. Lemma 4). Clearly $\eta$ is an $\operatorname{Aut}(L)$ equivariant bijection from $F$ onto $\left(W(L) \cap \mathfrak{n}^{\perp}\right) / L$. In the following we assume that $\lambda \in \hat{L}-F$.

(b) Let us observe next that the space $\hat{N} / L$ is countably separated. Since $\zeta$ is a homeomorphism from $\hat{N}$ onto $n^{\prime} / N$ (cf. [4, Theorem, p. 409]), to this end it is enough to show that any $L$ orbit on $\mathfrak{n}^{\prime}$ is closed. By assumption there is a compact subalgebra $\mathfrak{b}$ of $\delta$ such that $\delta$ is equal to the direct sum of subspaces $\mathfrak{h}+\mathfrak{n}$. Hence $H=\exp (\mathfrak{h})($ A.4) is compact in $L$ and we also have $L=H N$. Therefore to obtain the desired conclusion, it suffices to recall that any $N$ orbit on $\mathfrak{n}^{\prime}$ is closed (cf. e.g. [18, Proposition 1, p. 153]). From this we infer that any $\lambda \in \hat{L}$, when restricted to $N$, is decomposable over a welldetermined $L$ orbit in $\hat{N}$, which we denote by $O(\lambda)$. Observe that if $\alpha \in$ $\operatorname{Aut}(L)$, clearly $O(\alpha \lambda)=\alpha O(\lambda)$. Let $o(\lambda) \subset \mathfrak{n}^{\prime}$ be the inverse image of $\zeta(O(\lambda))$; then $o(\alpha \lambda)=\alpha o(\lambda)$. This being said, we shall carry out the substance of the proof of our lemma in the following steps. We assign to each 
$f \in o(\lambda)$ an $L_{f}$ orbit $\varepsilon(f) \subset W(L)$ (cf. (c) below), and show that if $\alpha \in$ $\operatorname{Aut}(L)$, and $\varepsilon_{1}$ stands for the analogous procedure corresponding to $\alpha \lambda$, then $\varepsilon_{1}(\alpha f)=\alpha \varepsilon(f)$ (cf. (d) below). This being so, $\eta(\lambda)$ will be defined by

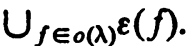

(c)(1) Let $f$ be a fixed element of $o(\lambda)(f \neq 0)$ and $\pi \in \hat{N}$ be such that $\zeta(\pi)=N f$; thus $O(\lambda)=L \pi \subset \hat{N}$. Let $\pi^{\prime}$ be a projective extension of $\pi$ to $L_{\pi}$ (A.2); we shall denote by the same symbol the corresponding representation of $L_{i}^{e}$ (cf. 1.1(c)). With notations as at the start of 2.3, let us write $\chi_{0}$ for the character of $\mathfrak{T}=\Psi(\mathscr{T})\left(\subset \mathfrak{R}^{4}\right)$ for which $\chi_{0}{ }^{\circ}(\Phi \mid T)$ is the conjugate of the identity map of the circle group $\mathrm{T} \subset L_{\pi}^{e}$ (cf. 1.1(c)) onto itself, and $\hat{\mathfrak{L}}_{-1}$ for the collection of all those elements of $\hat{\mathfrak{L}}$ which, on $\mathfrak{I}$, are multiples of $\chi_{0}$. If $\mu$ is some element of $\hat{\mathfrak{L}}_{-1}$, the representation $\tau=\pi^{\prime} \otimes(\mu \circ \Phi)$ of $L_{\pi}^{e}$ is equal to the identity operator on $T$; we denote by $\tau \sim$ the corresponding representation of $L_{\psi}$. We recall that by what we saw in (b) there is a well-determined $\mu$ as above such that, writing $\rho=\tau \sim$, we have $\lambda=\operatorname{ind}_{L \uparrow \uparrow} \rho$. (2) We again set $\mathfrak{Z}=\mathfrak{\Omega}_{0}$ (A.6) and define $\hat{\mathfrak{S}}_{-1}$ analogously to $\hat{\mathfrak{L}}_{-1} \cdot \mu \mid \mathfrak{Z}$ gives rise to a (finite) $\mathfrak{L}$ orbit, $\mathfrak{L} \sigma$ (say), in $\hat{\mathbb{S}}_{-1}$. Substituting 3 in place of $G$ in Lemma 4 , let us select an element $\kappa$ in $\delta(\sigma) \subset z^{\prime}$. We claim next that $\delta \Psi(\kappa)\left|\mathfrak{n}_{f}=f\right| \mathfrak{n}_{f}$. To see this it suffices to show that $\chi_{0} \circ\left(\Psi \mid \tau_{0}\right) \equiv \varphi_{f} \mid \vartheta_{0}$ (cf. 1.1(b)). This is clear by observing that, with the notations of $1.1(\mathrm{~d})$, we have $\Psi=\Phi \circ \gamma$ and

$$
\left(q\left(\pi^{\prime}\right) \circ \gamma\right)(n) \equiv\left(p(n), \overline{\varphi_{f}(n)}\right) \quad(n \in \mathscr{N}) .
$$

Since $\mathfrak{d}_{f} \cap \mathfrak{n}=\mathfrak{n}_{f}$, from this we conclude that there is an element $h$ of $\boldsymbol{d}^{\prime}$ such that $h \mid \delta_{f}=\delta \Psi(\kappa)$ and $h \mid \mathfrak{n}=f$. (3) Since by Lemma $4 \delta(\sigma) \subset W(3)$, Lemma 3 implies that $h \in W(L)$. (4) We claim that $L_{f} h$ is well determined by $f$ $(\in O(\lambda))$. In fact, if $\sigma_{1} \in \mathfrak{L} \sigma$ and $\kappa_{1} \in \delta\left(\sigma_{1}\right)$, by $\mathfrak{L}=\Psi(\mathfrak{L})$ there is an $s \in L_{f}$ such that $\kappa_{1}=s \kappa$. Putting $\delta_{\pi}=\mathfrak{n}+\delta_{f}$, we infer from this that $h_{1}\left|\delta_{\pi}=s h\right| \delta_{\pi}$. By Lemma 2, we have $N_{f} h=h+\delta_{\pi}^{+}$, whence clearly $L_{f} h=L_{f} h_{1}$. Summing up, we can define the $L_{f}$ orbit $\varepsilon(f)$ in $W(L)$ by $L_{f} h$, where $h \mid \delta_{f}=\delta \Psi(\kappa)$ (as above) and $h \mid \mathfrak{n}=f$.

(d) For a fixed $\alpha \in \operatorname{Aut}(L)$ let us put $f_{2}=\alpha$; as already observed (cf. (b) above) in this case $f_{2} \in o\left(\lambda_{2}\right)\left(\lambda_{2}=\alpha \lambda_{1}, \lambda_{1}=\lambda\right)$. We also write $f_{1}=f$ and, in general, shall distinguish notions relative to $f_{j}$ by the index $j=1,2$. Denoting by $\varepsilon_{j}$ the procedure of (c), corresponding to $\lambda_{j}$ and $f_{j}$, we claim that $\varepsilon_{2}\left(f_{2}\right)=$ $\alpha\left(\varepsilon_{1}\left(f_{1}\right)\right)$. To simplify notations, if, respectively, $\pi=\pi_{j}(j=1,2)$ we shall write $L_{j}=L_{\pi}$ and $D_{j}=L_{\pi}^{e}$. Since $f_{2}=\alpha f_{1}$ and $N f_{j}=\zeta\left(\pi_{j}\right)$ we have $\pi_{2}=\alpha \pi_{1}$ and thus $L_{2}=\alpha\left(L_{1}\right)$. Let us put $N_{j}$ for $N \subset D_{j}$ (cf. 1.1(c)). (1) Assuming that $\beta$ has the meaning as in Lemma 1 ( $\mathcal{G}$ loc. cit. replaced by $\mathfrak{L}$ ) with the notations of the diagram at the start of 2.3 , we claim that there is an isomorphism $\omega$ from $D_{1}$ onto $D_{2}$ such that

$$
\omega\left(N_{1}\right)=N_{2}, \alpha \circ h_{1}=h_{2} \circ \omega \text { and } \omega \circ \gamma_{1}=\gamma_{2} \circ \beta \text {. }
$$


In fact, let $\pi_{1}^{\prime}$ be a projective extension of $\pi_{1}$ to $L_{1}$, and let us put $\pi_{2}^{\prime}=\alpha \pi_{1}^{\prime}$. Clearly, if $\xi_{j}$ is the cocycle corresponding to $\pi_{j}^{\prime}$, we have

$$
\xi_{2}(\alpha(a), \alpha(b)) \equiv \xi_{1}(a, b) \quad\left(a, b \in L_{1}\right) .
$$

In this fashion, using the notations of 1.1(c), we can define an isomorphism $\tau$ : $\mathbb{B}\left(\pi_{1}^{\prime}\right) \rightarrow \mathbb{B}\left(\pi_{2}^{\prime}\right)$ by $\tau((a, u))=(\alpha(a), u)\left(a \in L_{1}, u \in T\right)$. Putting $q_{j}=q\left(\pi_{j}^{\prime}\right)$ : $D_{j} \rightarrow \mathbb{S}\left(\pi_{j}\right)$ (cf. loc. cit.) we write $\omega=q_{2}^{-1} \circ \tau \circ q_{1}$. This is an isomorphism, depending on $\alpha$ only, of $D_{1}$ onto $D_{2}$ and we are going to show that it possesses all the properties claimed above. Since we clearly have $\omega\left(N_{1}\right)=N_{2}$ and $\alpha \circ h_{1}=h_{2} \circ \omega$, to this end it is enough to prove that $\omega \circ \gamma_{1}=\gamma_{2} \circ \beta$. As in 1.1(d), we assume that $k_{j}: \mathscr{L}_{j} \rightarrow T$ is determined by

$$
V_{j}\left(a_{j}\right)=k_{j}\left(a_{j}\right) \pi_{j}^{\prime}\left(p_{j}\left(a_{j}\right)\right) \quad\left(a_{j} \in \mathfrak{L}_{j} ; j=1,2\right) .
$$

Defining $\eta_{j}: \mathscr{L}_{j} \rightarrow \mathbb{S}\left(\pi_{j}^{\prime}\right)$ by $\eta_{j}\left(a_{j}\right)=\left(p_{j}\left(a_{j}\right), k_{j}\left(a_{j}\right)\right)$ we recall (cf. loc. cit.) that $\gamma_{j}={ }_{q}^{-1} \circ \eta_{j} \circ \eta_{j}(j=1,2)$. Hence it will suffice to establish that $\tau \circ \eta_{1}=\eta_{2} \circ \beta$. Let us assume now that $a_{2}=\beta\left(a_{1}\right) \in e_{2}$. Since $\left(\tau \circ \eta_{1}\right)\left(a_{1}\right)=\left(\alpha\left(p_{1}\left(a_{1}\right)\right)\right.$, $\left.k_{1}\left(a_{1}\right)\right),\left(\eta_{2} \circ \beta\right)\left(a_{1}\right)=\left(p_{2}\left(a_{2}\right), k_{2}\left(a_{2}\right)\right)$ and (cf. end of (a), proof of Lemma 1) $\alpha\left(p_{1}\left(a_{1}\right)\right)=p_{2}\left(\beta\left(a_{1}\right)\right)=p_{2}\left(a_{2}\right)$, it will be enough to show that $k_{1}\left(a_{1}\right)=$ $k_{2}\left(a_{2}\right)$. It is to obtain this conclusion that we use Lemma 1 by observing that it yields $V_{2}\left(a_{2}\right)=\left(\beta V_{1}\right)\left(\beta\left(a_{1}\right)\right)=V_{1}\left(a_{1}\right)$, whence the result is clear by $\pi_{1}^{\prime}\left(p_{1}\left(a_{1}\right)\right)=\pi_{2}^{\prime}\left(p_{2}\left(a_{2}\right)\right)$. (2) We infer from (1) that there is a surjective isomorphism $g: \mathfrak{L}_{1} \rightarrow \mathfrak{R}_{2}$ such that $g \circ \Psi_{1}=\Psi_{2} \circ \beta$. In fact, by $\omega\left(N_{1}\right)=N_{2}$ there is a $g$ of the said kind verifying $g \circ \Phi_{1}=\Phi_{2} \circ \omega$, whence we obtain that

$$
g \circ \Psi_{1}=g \circ \Phi_{1} \circ \gamma_{1}=\Phi_{2} \circ \omega \circ \gamma_{1}=\Phi_{2} \circ \gamma_{2} \circ \beta=\Psi_{2} \circ \beta,
$$

or that $g \circ \Psi_{1}=\Psi_{2} \circ \beta$, which is the desired conclusion. (3) We recall that $\lambda_{1}=\lambda, \lambda_{2}=\alpha \lambda_{1}$ and that, by (c), $\lambda_{1}=\operatorname{ind}_{L_{1} \uparrow L} \rho_{1}$. From here we obtain at once that $\lambda_{2}=\operatorname{ind}_{L_{2} \uparrow L} \rho_{2}$, where $\rho_{2}=\alpha \rho_{1}$ (A.3). We also have (cf. loc. cit.) $\rho_{1}=\left[\pi_{1}^{\prime} \otimes\left(\mu_{1} \circ \Phi_{1}\right)\right]^{\sim}$ which, by $\alpha \circ h_{1}=h_{2} \circ \omega$ and (2), implies at once that $\rho_{2}=\left[\pi_{2}^{\prime} \otimes\left(g \mu_{1} \circ \Phi_{2}\right)\right]^{\sim}$. (4) As a corollary of the reasoning of Lemma 4, we have the following result. Suppose that $G_{j}$ is compact, connected, and that $\alpha$ : $G_{1} \rightarrow G_{2}$ is an isomorphism onto. Then

$$
\alpha\left(\delta_{1}(\sigma)\right)=\delta_{2}(\alpha \sigma) \quad\left(\sigma \in \hat{G}_{1}\right) .
$$

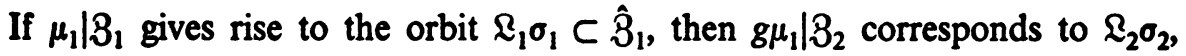
where $\sigma_{2}=g \sigma_{1}$. Assume that $\kappa_{1} \in \delta_{1}\left(\sigma_{1}\right)$; by what we have just said this implies $g \kappa_{1} \in \delta_{2}\left(\sigma_{2}\right)$. Let us put $\kappa_{2}=g \kappa_{1}$ and denote by $\delta_{j}$ the Lie algebra of $L_{j}$ if $f=f_{j}(j=1,2)$. If $h \in \varepsilon_{1}\left(f_{1}\right)$ is such that $h \mid \delta_{1}=\delta \Psi_{1}\left(\kappa_{1}\right)$ then, by $\Psi_{2} \circ \beta=g \circ \Psi_{1}$ (cf. (2)) and $p_{2} \circ \beta=\alpha \circ p_{1}$, we also have $h \mid \delta_{2}=\delta \Psi_{2}\left(\kappa_{2}\right)$. Since at the same time $h \mid \mathfrak{n}=f_{1}$ and, hence, $\alpha h \mid \mathfrak{n}=f_{2}$, by what we saw, respectively, in (c) and (3) above, we conclude that $\alpha h \in \varepsilon_{2}\left(f_{2}\right)$, completing the proof of $\varepsilon_{2}\left(f_{2}\right)=\alpha\left(\varepsilon_{1}\left(f_{1}\right)\right)$. 
(e) We infer from our previous discussion that the set $\bigcup_{f \in o(\lambda)} \varepsilon(f)$ is an $L$ orbit on ' $\boldsymbol{\delta}^{\prime}$, well determined by $\lambda \in \hat{L}-F$; we shall denote it by $\eta(\lambda)$. Since, by (c)(3), $\varepsilon(f) \subset W(L)$, we have $\eta(\lambda) \in W(L) / L$.

(f) Next we prove that $\eta: \hat{L} \rightarrow W(L) / L$, as defined, respectively, in (a) and (e), is surjective. Suppose that $O \in W(L) / L$; we shall construct $\lambda \in \hat{L}$ with $\eta(\lambda)=O$. By what we saw in (a) we can assume that $O \mid \mathfrak{n} \neq\{0\}$. Hence, if $h$ is some fixed element in $O$, and $f=h \mid \mathfrak{n}$, we have $f \neq 0$. This being so, to obtain $\lambda$ of the required kind it suffices to reverse the procedure of (c) as follows. Let $\kappa \in z^{\prime}$ be such that $\delta \Psi(\kappa)=h \mid \delta_{f}$. Since $h \in W(L)$, we conclude by Lemma 3 that $\kappa \in W(3)$. Hence, by Lemma 4 , there is a $\sigma \in \hat{3}$ satisfying $\delta(\sigma)=3 \kappa$. By what we saw in (c)(2) above, we even have $\sigma \in \hat{3}_{-1}$. Let $\mu \in \hat{\mathfrak{L}}_{-1}$ be such that, when restricted to 3 , it gives rise to the orbit $\mathfrak{L} \sigma \subset \hat{\mathbb{Z}}$. We determine $\pi \in \hat{N}$ (A.2) by $\zeta(\pi)=N f$ and denote by $\pi^{\prime}$ a projective extension of $\pi$ to $L_{\boldsymbol{r}}$. We form, similarly as in (c)(1), $\rho=\left[\pi^{\prime} \otimes(\mu \circ \Phi)\right]^{-}$and set $\lambda=\operatorname{ind}_{L_{\uparrow} \uparrow} \rho$. We have $\lambda \in \hat{L}$ and, clearly, $\eta(\lambda)=O(=L h)$.

(g) Let us assume now that $\eta\left(\lambda_{1}\right)=\eta\left(\lambda_{2}\right)$. If, in addition, the kernel of at least one of these representations contains $N$, we infer from (a) that $\lambda_{1}=\lambda_{2}$, since, by (c), $\operatorname{ker}(\lambda) \not X$ implies $\eta(\lambda) \not \subset \mathfrak{n}^{\perp}$. We suppose now that $\lambda_{j} \in \hat{L}-$ $F$. Since, for any $\lambda \in \hat{L}, \eta(\lambda) \mid \mathfrak{n}=o(\lambda)$, our assumption yields that $o\left(\lambda_{1}\right)=$ $o\left(\lambda_{2}\right)$. Let $f$ be a fixed element of either side and suppose that $\zeta(\pi)=N f$. Then with notations as in (c)(1), we can write $\lambda_{j}=\operatorname{ind}_{L_{\uparrow} \uparrow L} \rho_{j}$, where $\rho_{j}=\left[\pi^{\prime}\right.$ $\left.\otimes\left(\mu_{j} \circ \Phi\right)\right]^{\sim}(j=1,2)$. Our assumption further entails that $\mu_{1}$ and $\mu_{2}$, when restricted to 3 , give rise to the same orbit $\& \sigma \subset \hat{\mathcal{B}}_{-1}$. Since $\& / \mathcal{Z}$ is finite, the collection of all such elements of $\hat{L}$ is also finite. Summing up, if $\eta\left(\lambda_{1}\right)=$ $\eta\left(\lambda_{2}\right)=O$ (say), $\lambda_{j}$ belongs to a finite subset, determined by $O$, of $\hat{L}$. Evidently, if $\delta$ is nilpotent $\eta$ coincides with the Kirillov isomorphism, completing the proof of Lemma $6 . \quad$ Q.E.D.

REMARK 3. Let us note that the map $\eta: \hat{L} \rightarrow W(L) / L$ of our lemma is uniquely determined by the following properties: (1) $\eta \mid F \equiv \delta$ (cf. (a)); (2) if $\lambda \in \hat{L}-F$ then: (A) $\eta(\lambda) \mid \mathfrak{n}=o(\lambda)$ (cf. (b)); (B) if $f \in o(\lambda), \zeta(\pi)=N f$ and if we write, as in (c)(1), $\lambda=\operatorname{ind}_{L_{*} \uparrow L} \rho, \rho=\left[\pi^{\prime} \otimes(\mu \circ \Phi)\right]^{\sim}$, denote by $\mathfrak{L} \sigma$ the orbit of $\mu \mid 3$, and take $\kappa \in \delta(\sigma)$, then there is an $h \in \eta(\lambda)$ such that $h \mid \mathfrak{n}=f$ and $h \mid \delta_{f}=\delta \Psi(\kappa)$ (cf. (c)(2)). In fact, let us put $\eta_{1}=\eta$ and assume that $\eta_{2}$ : $\hat{L} \rightarrow W(L) / L$ also has the above properties; we are going to prove that $\eta_{1}=\eta_{2}$. It is clear that $\eta_{1}\left|F=\eta_{2}\right| F$. If $\lambda \in \hat{L}-F$, we first have, by (2A), $\eta_{1}(\lambda)\left|\mathfrak{n}=o(\lambda)=\eta_{2}(\lambda)\right| \mathfrak{n}$. Let $f$ be a fixed element in $o(\lambda)$; note that now $f \neq 0$. Let us assume that $\sigma_{j}$ are elements of $\hat{\beta}$ in the orbit, well determined by $\lambda$ and $f$, of $\mu \mid 3$, and that $\kappa_{j} \in \delta\left(\sigma_{j}\right)(j=1,2)$. By (2B) there are elements $h_{j} \in \eta_{j}(\lambda)$ such that $h_{j} \mid \mathfrak{n}=f$ and $h_{j} \mid \delta_{f}=\delta \psi\left(\kappa_{j}\right)(j=1,2)$. Hence to show that $\eta_{1}(\lambda)=\eta_{2}(\lambda)$ it suffices to find an $s \in L_{f}$ with $h_{2}=s h_{1}$. As in (c)(4) above, there is a $t \in L_{f}$ such that $\kappa_{2}=t \kappa_{1}$ implying $t h_{1}\left|b_{f}=h_{2}\right| b_{f}$. Thus also $t h_{1} \mid \delta_{\pi}=$ 
$h_{2} \mid \delta_{\pi}\left(\delta_{\pi}=\mathfrak{n}+\delta_{f}\right)$. Using Lemma 2 and reasoning as loc. cit. we can determine $n \in N_{f}$ such that $h_{2}=n t h_{1}$, and therefore we can take $s=n t \in L_{f}$.

In the following statement we call an orbit of $L$ on $\delta^{\prime}$ admissible if it is contained in $W(L)$. Let $G$ be some topological group, $j: T \rightarrow G^{\natural}$ (A.7) an injection of the circle group T, and $\chi_{0}$ the character of $j(T)$ such that $\chi_{0}{ }^{\circ} j$ is the identity map of $T$ onto itself. We shall call a unitary representation of $G$, the restriction of which to $j(T)$ is a multiple of $\chi_{0}$, a class one representation, and denote by $\hat{G}_{1}$ the corresponding part of $\hat{G}$. Analogously, we write $\hat{G}_{-1}$ for the part of $\hat{G}$ obtained by replacing above $\chi_{0}$ through $\bar{\chi}_{0}$ (cf. also (c)(1) in the proof of Lemma 6). Given a set $S$, below we shall denote by $\#(S)$ its cardinality. Observe that $\#\left(\hat{G}_{1}\right)=\#\left(\hat{G}_{-1}\right)$.

Proposition 1. Suppose that $L$ is a connected and simply connected Lie group, the Lie algebra $\delta$ of which is nilpotent and cocompact. Then: (i) There is a canonical surjection $\eta$, equivariant with respect to $\operatorname{Aut}(L)$, from the dual of $L$ onto the set of all admissible orbits in $D^{\prime}$. If $L$ is nilpotent, $\eta$ coincides with the Kirillov isomorphism. (ii) Each admissible orbit $O$ determines an element of $H^{2}\left(\pi_{1}(O), T\right)$ (to be denoted by [O]) such that if $\Lambda$ is the corresponding central extension of the fundamental group $\pi_{1}(O)$ through the circle group, the number of elements in $\hat{L}$, the image of which is $O$, is equal to the number of class one elements in the dual of $\Lambda$. In particular, it does not exceed the order of $\pi_{1}(O)$. (iii) Assume that $E$ is an arbitrary finite group and $\alpha \in H^{2}(E, T)$. There is a group of our class with an abelian radical and having an admissible orbit $O$ such that $\pi_{1}(O)=E$ and $[O]=\alpha$.

Proof. Ad (i). This we have already proved in Lemma 6 and Remark 3. Ad (ii) Assume that $O$ is a given admissible orbit. (a) If $O \subset \mathfrak{n}^{\perp}$, by virtue of our definition of $\eta$ (cf. (a), loc. cit.) $\lambda \in \hat{L}$ is well determined by $\eta(\lambda)=O$. But at the same time (cf. (1) in 2.1) if $h \in O, L_{h}$ is connected. (b) We suppose next that $O \mid \mathfrak{n} \neq\{0\}$. Let $h$ be a fixed element of $O$ and $f=h \mid \mathfrak{n}$; hence $f \neq 0$. With notations similar to those of (c)(2), proof of Lemma 6, we assume $\kappa \in z^{\prime}$ such that $h \mid \delta_{f}=\delta \Psi(\kappa)$ and $\sigma \in \hat{3}$ with $3 \kappa=\delta(\sigma)$. (1) Let us write $\mathbb{Q}$ for the set of all elements of $\hat{\mathfrak{L}}$ which, when restricted to $\mathcal{Z}$, give rise to the orbit $\mathfrak{\delta} \subset \hat{\mathfrak{B}}$. By what we saw in (g) loc. cit., we conclude that $\#\left(\bar{\eta}^{-1}(O)\right)=$ $\#(\mathfrak{Q})$. (2) Let us now replace $\mathfrak{M}$ by $\mathfrak{L}$ in the diagram of Remark 1 above. If 9 stands for the set of all those elements of $\hat{\mathfrak{L}}_{\sigma}$ which on 3 are multiples of $\sigma$, reasoning as in (c)(1) above, we conclude that $\#(\mathscr{B})=\#\left(\hat{\Gamma}_{-1}\right)=\#\left(\hat{\Lambda}_{1}\right)$. (3) With notations as in (A), proof of Lemma 3, and setting $\mathscr{N}=\operatorname{ker}(\chi) \subset \mathfrak{N}$, we show next that $\Gamma=L_{h} N / \dot{M}$. Let us observe at once that by so doing we shall have settled (ii). In fact, division of $\Gamma$ by $\Re / \Upsilon^{\circ} \sim T$ will then yield that

$$
\mathfrak{L}_{h} \Re /\left(\mathfrak{C}_{h}\right)_{0} \Re=L_{h} N_{f} /\left(L_{h}\right)_{0} N_{f}
$$


which, by $L_{h} \cap N_{f}=N_{h}=\left(L_{h}\right)_{0} \cap N_{f}$, is equal to $L_{h} /\left(L_{h}\right)_{0}=\pi_{1}(O)$. In this manner $\Lambda$, up to isomorphism well determined by $O$, turns out to be a central extension of $\pi_{1}(O)$ by the circle group. To establish our previous statement, let us recall first that (cf. loc. cit.) $\Psi^{-1}\left(\mathfrak{R}_{k}\right)=\mathfrak{L}_{h} \Re$ and thus (cf. Remark 1), putting $\mu=\psi \circ(\Psi \mid \Re)$, we get $\mu\left(\mathscr{L}_{h} \Re\right)=\psi\left(\mathfrak{R}_{k}\right)=\Gamma$. Hence it suffices to show that $\operatorname{ker}(\mu)=\mathscr{0}$. But this follows at once from $\operatorname{ker}(\psi)=\dot{3}_{\kappa}$ and $\chi=\chi_{\kappa} \circ(\Psi \mid \Im)$. Before proceeding, we wish to make the following two comments. (A) Let us set

$$
\tilde{\mathfrak{L}}_{h}={ }^{-1}\left(\left(L_{h}\right)_{0}\right)=\left(\mathscr{L}_{h}\right)_{0} \cup \varepsilon\left(\mathscr{L}_{h}\right)_{0} \text { and } \stackrel{\mathfrak{E}}{h}_{h}=\operatorname{ker}\left(\chi \mid \tilde{\mathscr{L}}_{h}\right) \text {. }
$$

We claim that if $\chi_{h} \neq 1$, we have $\Gamma=\mathscr{L}_{h} / \mathfrak{E}_{h}$. In fact, putting $v=\psi \circ\left(\Psi \mid \mathfrak{L}_{h}\right)$ $=\mu \mid \mathscr{L}_{h}$, let us first observe that $\nu\left(\mathscr{L}_{h}\right)=\Gamma$. To see this it is enough to show that $\psi(\Psi(\mathscr{T})) \subset \nu\left(\left(\mathcal{L}_{h}\right)_{0}\right)$. If $n \in \Re$, by $\chi_{n} \neq 1$ there is an $a \in\left(\mathscr{L}_{h}\right)_{0}$ such that $\chi(n)=\chi(a)$ or $n=a m(m \in \mathscr{T} R)$, whence $\psi(\Psi(n))=\mu(a) \in \nu\left(\left(\mathcal{E}_{h}\right)_{0}\right)$. On the other hand, by $\Re \cap \mathfrak{L}_{h}=\tilde{\mathfrak{L}}_{h}$ we have

$$
\operatorname{ker}(\nu)=\operatorname{ker}(\mu) \cap \mathfrak{L}_{h}=\mathfrak{\pi} \cap \mathfrak{L}_{h}=\operatorname{ker}\left(\chi \mid \tilde{E}_{h}\right)=\mathfrak{L}_{h},
$$

proving our statement. (B) Let us assume that there is a $\varphi \in X\left(\mathscr{L}_{h}\right)$ such that $\varphi \mid\left(\mathcal{L}_{h}\right)_{0} \equiv 1$ and $\varphi(\varepsilon)=-1$. We observe that by what we saw in 1.1(a) this is, in particular, always the case whenever $\mathfrak{n}$ is abelian. Also our assumption implies that there is a $\chi_{h} \in X\left(\left(L_{h}\right)_{0}\right)$ with $d \chi_{h}=i\left(h \mid \delta_{h}\right)$. Putting $\dot{L}_{h}=\operatorname{ker}\left(\chi_{h}\right)$, one easily verifies that $L_{h} / \dot{L}_{h}=L_{h} / \dot{L}_{h}$. Summing up, by what we have just seen we conclude that if $\mathfrak{n}$ is abelian, and $h \in W(L)$ is such that $\chi_{h} \mid\left(L_{h}\right)_{0} \neq 1$ $\left(\chi_{h} \equiv \varphi_{h}\right)$, then $\Gamma=L_{h} / \dot{L}_{h}$. Ad (iii) Let us suppose that $E$ is a given finite group and that $\Lambda$ satisfies the exact sequence

$$
1 \rightarrow \mathrm{T} \stackrel{\text { central }}{\rightarrow} \Lambda \rightarrow E \rightarrow 1 .
$$

By what we have just seen, to settle this point it is enough to find an $L$ of our class for which $\mathfrak{n}$ is abelian and an $h \in W(L)$ with $L_{h} / \dot{L}_{h}=\Lambda$. (1) We start by observing that one can assume $\Lambda \subset G$, where $G$ is semisimple and simply connected. In fact, to this end it suffices, for instance, to consider a faithful linear representation of $\Lambda$ in $S U(n)$. (2) Next we note that given any closed subgroup $K \subset G$, there is a representation $\psi$ of $G$ by orthogonal transformations on a Euclidean space $V$ and a $v \in V$ such that $G_{v}=K$ (A.1). In fact, for a suitable integer $n$, there is a regular and $G$ equivariant imbedding $f$ of $G / K$ into $S^{n} \subset E^{n+1}$ (cf. [22, 5.10.11, p. 143]); hence it suffices to take $V=E^{n+1}$ and $v=f(K)$. Thus, in particular, we can assume $G_{v}=\Lambda$. (3) Putting $a u$ in place of $\psi(a) u(a \in G, u \in V)$ we define

$$
L=\{(a, u) ; a \in G, u \in V \text { with }(a, u)(b, v)=(a b, u+a v)\} .
$$

Writing $L=\exp (\S), G=\exp (g)$ (A.4) we have a direct sum decomposition 
$\mathfrak{d}=\mathfrak{g}+\mathfrak{n}$, where $\mathfrak{n}$ is identifiable to $V$. Setting, as we can, $\mathfrak{g}^{\prime}=\mathfrak{n}^{\perp}, \mathfrak{n}^{\prime}=\mathfrak{g}^{\perp}$, we also obtain the $G$ invariant decomposition $\mathfrak{d}^{\prime}=\mathfrak{g}^{\prime}+\mathfrak{n}^{\prime}$. We identify $g$ to $g^{\prime}$ $\left(\mathfrak{n}\right.$ to $\mathfrak{n}^{\prime}$ ) by aid of the Killing form $B$ on $\mathfrak{g} \times \mathfrak{g}$ (resp. orthogonal metric (, ) on $V)$. (4) Let $l \in g$ be such that $\Lambda_{0}=\{\exp (t l) ; t \in R\}$ and $\exp (t l)=$ unity if and only if $t=2 \pi n(n \in Z)$. We put $h=(l / B(l, l))+v$ and claim that $L_{h}=\Lambda N_{h}$. In fact, since $f=h \mid n=v$, we first have $L_{f}=\Lambda N$. On the other hand, by $\Lambda_{0}=\Lambda^{\natural}$, for any $\delta \in \Lambda: \delta l=l$ and hence $\Lambda \subset L_{h}$. In this fashion $L_{h}=\left(L_{f}\right)_{h}=\Lambda N_{h}$. (5) We observe next that $h \in W(L)$. In fact, let us note first that $\left(L_{h}\right)_{0}=\Lambda_{0} N_{h}$. We set $\chi_{h}^{\prime}(\exp (l)) \equiv \exp (i(l, h))\left(l \in \mathfrak{n}_{h}\right)$ and note that the map $\chi_{h}:\left(L_{h}\right)_{0} \rightarrow \mathrm{T}$ determined by

$$
\chi_{h}(\exp (t l) \cdot n)=\exp (i t) \cdot \chi_{h}^{\prime}(n) \quad\left(n \in N_{h}\right)
$$

is a character of $\left(L_{h}\right)_{0}$ satisfying $d \chi_{h}=i\left(h \mid \delta_{h}\right)$. Since $\mathfrak{n}$ is abelian, this proves that $h \in W(L)$. (6) Let us show finally that $L_{h} / \dot{L}_{h}=\Lambda$. We can realize $\Lambda$ as the set of all pairs $(a, u)(a \in E, u \in T)$ with the law of composition $(a, u)(b, v)=(a b, \alpha(a, b) u v)$. Writing $b$ also for $(b, 1) \in \Lambda$, we then have setwise $L_{h}=E \Lambda_{0} N_{h}$. This being so, one verifies immediately that the map $F$ : $L_{h} \rightarrow \Lambda$ defined by $F(a u m)=\left(a, u \chi_{h}(m)\right)$ is a homomorphism onto such that $\operatorname{ker}(F)=\dot{L}_{h}$, completing the proof of Proposition 1. Q.E.D.

2.4. From now on we shall assume that $G$ is a connected and simply connected Lie group with the Lie algebra $g$ such that the radical of $g$ is cocompact. Denoting again by $\mathfrak{n}$ the nilradical, we set $\mathfrak{b}=[\mathfrak{g}, \mathfrak{g}]+\mathfrak{n}$ and observe that its radical, equal to $\mathfrak{n}$, is cocompact. In fact, any Levi section $\mathfrak{h}$ for $\mathfrak{g}$ is such for $\mathfrak{d}$ too; but by our hypothesis bearing on $\mathfrak{g}, \mathfrak{h}$ is necessarily compact. We set $L=\exp (\mathfrak{d})$ (A.4).

LEMMA 7. With the above notations we have $W(G) \mid \delta=W(L)$.

Proof. (A) Let $g \in \mathfrak{g}^{\prime}$ be such that $g \mid \mathfrak{d}=h \in W(L)$; we are going to prove that $g \in W(G)$. Putting $f=g \mid \mathfrak{n}, \mathcal{L}=p\left(L_{f}\right) \subset \mathcal{G}$ (cf. 1.1(a)) and $\mathscr{D}=\left(L_{h}\right)_{0}$ we recall that our assumption is equivalent to the existence of a $x_{h} \in X(D)$ such that $d x_{h}=i\left(h \mid \delta_{h}\right)$ and, if $\varepsilon \in \mathscr{D}$, also $\chi_{h}(\varepsilon)=-1$. Let us write $\mathcal{C}=$ $\exp \left(\delta_{g}\right) \subseteq \mathcal{G}$ (A.1, A.4); we have $\mathcal{C} \subseteq \mathscr{D}$. Setting $\psi=x_{h} \mid \mathcal{C}, \mathcal{C}=\operatorname{ker}(\psi)$ and $\mathcal{X}=\left(G_{g}\right)_{0}$ we shall show that $[\mathcal{K}, \mathcal{H}] \subseteq \dot{C}$ and that $\mathcal{X} / \mathcal{C}$ is isomorphic to a vector group. This will first imply the existence of an $\omega \in X(\mathcal{H C})$ with $\omega \mid \mathcal{C}=\psi$. Since $d \psi=i\left(g \mid \delta_{g}\right)$, replacing, if necessary, $\omega$ through $\zeta \omega$, where $\zeta \in X(H)$ such that $\zeta \mid \mathcal{E} \equiv 1$ is appropriately chosen, we shall be able to conclude that there is a $\chi_{g} \in X(\mathcal{H})$ satisfying $d \chi_{g}=i\left(g \mid g_{g}\right)$ and, if $\varepsilon \in \mathcal{H}$, also $\chi_{g}(\varepsilon)=-1$. To prove our previous assertion we first observe that [ $\mathcal{H}$, $\mathcal{H C}]=\exp \left(\left[g_{g}, g_{g}\right)(A .4)\right.$ and that $\left[g_{g}, g_{g}\right] \subset g_{g} \cap \delta=\delta_{g}$; hence $[\mathcal{K C}, \mathcal{F C}] \subset \mathcal{C}$. Thus it suffices to note that if $x, y \in g_{g}$, we have

$$
\psi(\exp ([x, y]))=\exp [i([x, y], h)]=1 .
$$


Since $G$ is simply connected, $G / L$ is isomorphic to a vector group and hence so is $\left(G_{g}\right)_{0} /\left(\left(G_{g}\right)_{0} \cap L\right)$. Thus $\left(G_{g}\right)_{0} \cap L$ is connected and equal to $\left(L_{g}\right)_{0}$. Therefore $\left(G_{g}\right)_{0} /\left(L_{g}\right)_{0}$ and also $\mathcal{X} / \mathcal{C}$ is isomorphic to some vector group. If $\varepsilon \in \mathcal{C}$ we clearly have $\varepsilon \in \mathcal{C}$, and thus $\chi_{g}(\varepsilon)=\psi(\varepsilon)=\chi_{h}(\varepsilon)=-1$, completing the proof of our statement.

(B) Let us now select $g$ in $W(G)$; we are going to show that $g \mid \delta=h \in$ $W(L)$. We start by observing that $\left[\delta_{h}, \delta_{h}\right] \subseteq g_{h}$. To this end it is enough to note that if $l \in g$ and $x, y \in \mathfrak{d}_{h}$ are arbitrary, we have

$$
([l,[x, y]], h)=([[l, x], y], h)+([x,[l, y]], h)
$$

and that, by $[g, g] \subseteq \delta$, both summands on the right-hand side vanish. $C$ being as in (A) we now set $\stackrel{\mathrm{C}}{=}=\operatorname{ker}\left(\chi_{g} \mid \mathcal{C}\right)$. By what we have just seen we conclude that $[\mathscr{D}, \mathscr{D}] \subseteq \mathcal{E}$. Therefore, to complete the proof of our lemma it will be enough to show that $\mathscr{D} / \mathcal{C}$ is isomorphic to a vector group. In fact, in this case, similarly as in (A) above, we shall be able to infer first that there is a $\chi_{h} \in X(D)$ satisfying $d \chi_{h}=i\left(h \mid D_{h}\right)$. If, furthermore, $\varepsilon \in \mathscr{D}$, then $\varepsilon \in \mathcal{C} \subseteq$ $\mathcal{X}$, and thus $\chi_{h}(\varepsilon)=\chi_{g}(\varepsilon)=-1$. To prove our point we observe that by Lemma 2 setting $g_{\lambda}=\delta+g_{h}$ we have $\left(L_{h}\right)_{0} g=g+g_{\lambda}^{\perp}$. Therefore, writing $F(a)=a g-g\left(a \in\left(L_{h}\right)_{0}\right)$ we obtain a map $F:\left(L_{h}\right)_{0} \rightarrow g_{\lambda}^{\perp}$ which, by [g, g] $\subseteq \mathfrak{d}$, is a homomorphism of $\left(L_{h}\right)_{0}$ onto the underlying group of $g_{\lambda}^{1}$. From this we infer that $\left(L_{h}\right)_{0} \cap G_{g}$ is connected and hence is equal to $\left(L_{g}\right)_{0}$. In this fashion $\left(L_{h}\right)_{0} /\left(L_{g}\right)_{0}$ and also $\mathbb{Q} / \mathcal{C}$ is isomorphic to a vector group, completing the proof of our lemma. Q.E.D.

3. We continue to employ the assumptions made at the start of 2.4. As we recalled in the introductory remarks to this chapter (cf. also 3.4(5) below), in order that a primitive ideal $J$ of $C^{*}(G)$ be of type $I$, in particular, it is necessary that the action of $G$ on a space, depending on $J$, be smooth. The purpose of this point is to find an expression for this condition in terms of $\mathrm{g}^{\prime} / G$; the corresponding result is contained in Lemma 14 .

3.1. Let $g$ be a fixed element of $g^{\prime}$; as before we set $f=g \mid \mathfrak{n}$ and $h=g \mid \boldsymbol{b}$.

LeMma 8. We have $\left\{a ; a \in G_{f}, a h\left|\delta_{f}=h\right| \delta_{f}\right\}=G_{h} N_{f}$.

Proor. (a) If $a \in G_{h} N_{f}$, there is a $b \in N_{f}$ such that $a h=b h$. Again putting $\delta_{\pi}=\mathfrak{n}+\delta_{f}$ we recall (cf. Lemma 2) that $N_{f} h=h+\delta_{\pi}^{\perp}$ and therefore ahl$\delta_{f}$ $=b h\left|\delta_{f}=h\right| \delta_{f}$. (b) To prove the opposite inclusion let us assume that $a \in G_{f}$ and $a h\left|\delta_{f}=h\right| \delta_{j}$. Then we also have $a h\left|\delta_{\pi}=h\right| \delta_{\pi}$ and hence, reasoning as above, we can find an $n \in N_{f}$ such that $a h=n h$, proving that $a \in G_{h} N_{f}$. Q.E.D.

We want to assume from now on that $f \neq 0$ also; then, with $\pi \in \hat{N}$ satisfying $N f=\zeta(\pi)$, we can form the diagram of 1.1(d). We set, as in 2.4, $\mathfrak{L}={ }_{p}^{-1}\left(L_{f}\right) \subset \mathcal{G}$ and $\mathfrak{R}=\Psi(\mathfrak{L}) . \mathfrak{R}$ is a compact group and we again write 
$\mathfrak{S}_{0}=\boldsymbol{Z}=\exp (z)$. Putting $\Psi^{\prime}=\Psi \mid \mathcal{L}$, we choose $\kappa \in z^{\prime}$ such that $\delta \Psi^{\prime}(\kappa)=$ $h \mid \delta_{f}$. Let us assume also that $g \in W(G)$. Then by Lemma $7 h \in W(L)$, and by Lemma $3 \kappa \in W(3)$. With $\sigma \in \hat{3}$ such that $3 \kappa=\delta(\sigma)$, we can form the diagram of Remark 1 in 2.2 with $3 \subset \mathfrak{M}=\Psi(\mathcal{G})$. Combining there two diagrams we obtain

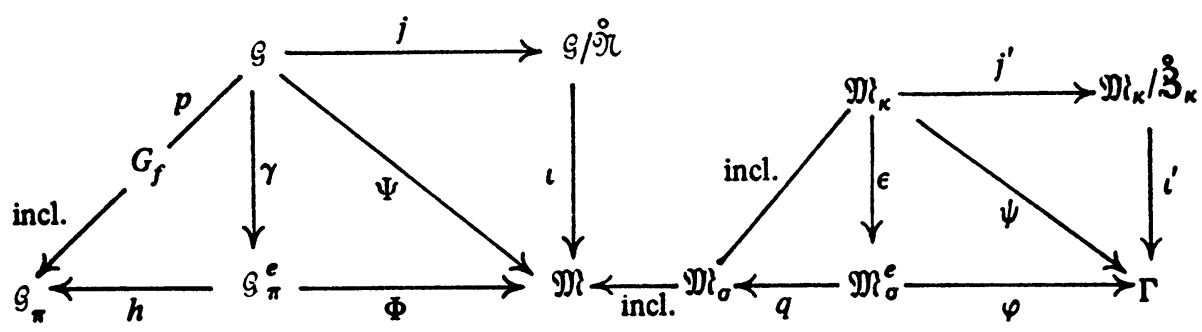

where incl stands for the inclusion map. Given a subset $A \subset G_{f}$, we shall write $A^{\prime}=p^{-1}(A)$. Putting $\delta=G_{h} N_{f}$, we infer from Lemma 9 that $\delta^{\prime}$ $=\Psi^{-1}\left(\mathfrak{D} R_{k}\right)$. Note that if $S_{0}=\exp (a)$ (cf. A.4), then we have $a=g_{h}+n_{f}$.

LEMMA 9. With the above notations we have

$$
\{a ; a \in \mathcal{S}, a(g \mid a)=g \mid \mathfrak{a}\}=G_{g}\left(L_{h}\right)_{0} N_{f} .
$$

Proof. (a) We write $\mathcal{T}$ for the left-hand side and start by observing that $N_{f} \subseteq \mathcal{T}$. In fact, putting $\mathrm{g}_{\pi}=\mathfrak{n}+\mathrm{g}_{f}$ we have, by Lemma $2, N_{f} g=g+g_{\pi}^{1}$. Hence the desired conclusion follows by noting that $g_{\pi} \supseteq a$. Thus it suffices to establish that $G_{h} \cap \mathcal{T} \subseteq G_{g}\left(L_{h}\right)_{0}$. Writing $g_{\lambda}=\mathfrak{d}+g_{h}$ we have, again by Lemma 2, $\left(L_{h}\right)_{0} g=g+g_{\lambda}^{1}$. If $a \in G_{h} \cap \mathcal{T}$, since $\delta+a=g_{\lambda}$, we also obtain $a g\left|g_{\lambda}=g\right| g_{\lambda}$. Thus there is a $l \in\left(L_{h}\right)_{0}$ such that $a g=l g$, implying $a \in$ $G_{s}\left(L_{h}\right)_{0}$.

(b) Let us put $\mathfrak{h}=g_{\pi}^{\perp}+g_{\lambda}^{\perp}$; by what we have just seen we can infer that $\left(L_{h}\right)_{0} N_{f} g=g+\mathfrak{h}$. Since clearly $\mathfrak{a} \subseteq g_{\lambda} \cap g_{\pi}$, in this manner $a \in G_{g}\left(L_{h}\right)_{0} N_{f}$ certainly implies $a(g \mid \mathfrak{a})=g \mid \mathfrak{a}$ and evidently also $a \in G_{h} N_{f}$. Q.E.D.

Let us put $\Lambda=\psi^{\circ}\left(\psi \mid \mathcal{S}^{\prime}\right)$; this is a homomorphism of $\mathcal{S}^{\prime}$ onto $\Gamma$.

Leman 10. We have $p\left(\Lambda^{-1}\left(\Gamma_{0}^{q}\right)\right)=\left(G_{g}\right)_{0}\left(L_{h}\right)_{0} N_{f}$.

Proof. (a) Let us write $V=\mathfrak{R}_{\sigma} / 3 ; \Gamma$ is a central extension, by the circle group, of $V$. We set $\mathfrak{F}=\mathfrak{\Omega}_{o} / \mathfrak{Z}$ and note that $\mathfrak{F}$ is finite and that $V / \mathfrak{F}$ is isomorphic to a closed subgroup of $R^{n}$ for $n$ sufficiently large. In fact, let us first observe that we have $V / \mathfrak{F}=\mathfrak{M}_{0} / \mathfrak{R}_{0}=\mathfrak{M}_{o} \mathfrak{L} / \mathfrak{R}$, and that the latter is closed in $\mathfrak{R} / \mathfrak{R}=G_{n} / L_{n}=G_{\pi} L / L$. Hence to arrive at the desired conclusion it is enough to remark that, since $L$ is the semidirect product of a compact and of a nilpotent group, $G_{\pi} L$ is closed in $G$ and, the latter being 
simply connected, $G / L$ is isomorphic to a vector group. In this manner, in particular, the maximal compact subgroup $T$ of $\Gamma_{0}$ is a circle group, such that $\Gamma_{0} / T$ is isomorphic to a vector gorup. We also have $\left[\Gamma, \Gamma_{0}\right] \subseteq T$. In fact, to see this it suffices to observe that $\left[V, V_{0}\right] \subseteq \mathfrak{F} \cap V_{0}$; hence, $F$ being finite, the right-hand side is trivial.

(b) Let us put $\Gamma_{0}=\exp (\gamma)$ and $\Gamma_{0} \supset T=\exp (\mathrm{R} v)$ (A.4). If $d$ is such that $\delta \Lambda(d)=g \mid \mathfrak{a}$, we evidently have $(v, d) \neq 0$. We denote by $\Gamma_{1}$ the centralizer, in $\Gamma$, of $\Gamma_{0}$ and claim that $\Gamma_{1}=\Gamma_{d}$. Clearly, to see this it is enough to show that $\Gamma_{d} \subseteq \Gamma_{1}$. If $l \in \gamma$ and $a \in \Gamma$, by $\left[\Gamma, \Gamma_{0}\right] \subseteq T$ we have $a l=l+c v$. If, furthermore, $a \in \Gamma_{d}$, we have $(l, d)=(a l, d)=(l, d)+c(v, d)$ and thus, by $(v, d) \neq 0, c=0$ and $a \in \Gamma_{1}$. Noting that the center of $\Gamma_{0}$ is connected, we infer from this that $\Gamma_{0}^{\natural}=\left(\Gamma_{1}\right)_{0}=\left(\Gamma_{d}\right)_{0}$. Let us next observe that, by virtue of Lemma 9, we have $p\left(\Lambda\left(\Gamma_{d}\right)\right)=G_{g}\left(L_{h}\right)_{0} N_{f}$. Hence, putting $M=\left(L_{h}\right)_{0} N_{f}$ and reasoning as in $A(\mathrm{a})$, proof of Lemma 3, we obtain $p\left(\Lambda^{-1}\left(\Gamma_{0}^{\natural}\right)\right)=\left(G_{g} M\right)_{0}$.

(c) We shall complete the proof of our lemma by showing that $M \cap G_{g}$ is connected. In fact, in this case $G_{g} \cap M=\left(G_{g}\right)_{0} \cap M$, and hence also $\left(G_{g} M\right)_{0}$ $=\left(G_{g}\right)_{0} M$ which, along with the relation at the end of $(\mathrm{b})$, yields the desired conclusion. Let us put $\Delta=G_{g} \cap M$ and, for $m \in M, f(m)=m g-g$. By what we saw in (b), proof of Lemma $9, f$ provides a continuous bijection from $M / \Delta$ onto a vector group $\mathfrak{h}$. It is easily seen to be a homeomorphism and thus $\Delta$ is connected. Q.E.D.

3.2. Let $\lambda$ be a fixed element of $\hat{L}$. We recall (cf. [18, Lemma 3.4, p. 483]) that if $\mathbb{Q}$ is the collection of all connected subgroups $A$ of $G$, such that $A \supseteq L$ and that there is a $\lambda^{\prime} \in \hat{A}$ which is $\left(G_{\lambda}\right)_{0}$ invariant and satisfies $\lambda^{\prime} \mid L=\lambda$, then $\mathbb{Q}$ admits a unique maximal element.

Leman 11. Assume that $g \in g^{\prime}$ is such that $g \mid \delta \in \eta(\lambda)$ (cf. Lemma 6). Then $\Pi=\left(G_{8}\right)_{0} L$.

Proof. (a) Let us assume first that $\operatorname{ker}(\lambda) \supseteq N$. Since $G / N$ is reductive, $\lambda$ extends to $G$ and thus $\Pi=G$. On the other hand, by what we saw in (a), proof of Lemma 6, $g \mid \mathfrak{n}=0$, implying $\left(G_{g}\right)_{0} L=G$.

(b) From now on we suppose that $\lambda \mid N$ is not trivial. In the following we shall make extensive use of the notations of the diagram of 3.1 with the following additional conventions. Given a projective extension $\pi^{e}$ of $\pi \in \hat{N}$ to $G_{\pi}$, we let $\pi^{e}$ stand also for the corresponding representation of $G_{\pi}^{e}$. If $M \subset G_{\pi}^{e}$ is some subgroup we shall write, whenever convenient, $\pi^{e}$ and $\Phi$ (cf. loc. cit.) in place of, respectively, $\pi^{e} \mid M$ and $\Phi \mid M$. Also, given a subset $K \subset G_{\pi}$, we denote by $K^{e}$ its inverse image in $G_{\pi}^{e}$; similar rules will be employed with regard to $\mathfrak{M} e_{\sigma}$.

(1) Proceeding as in (c)(1), proof of Lemma 6, we can write $\lambda=\operatorname{ind}_{L_{\uparrow} \uparrow} \rho$, 
where $\rho=\left[\pi^{e} \otimes(\mu \circ \Phi)\right]^{\sim}\left(\mu \in \hat{\mathfrak{L}}_{-1}\right.$ and $\pi^{e}, \Phi$ stands, respectively, for $\pi^{e} \mid L_{\pi}^{e}$ and $\left.\Phi \mid L_{\pi}^{e}\right)$. Again putting $3=\Omega_{0}$ (cf. (c)(2), loc. cit.) and assuming that $\mu \mid \mathcal{B}$ gives rise to the (finite) orbit $\Omega \sigma$ in $\hat{\mathbb{B}}$, where, as in 3.1 , we shall suppose $\delta(\sigma)=3 \kappa, \delta \Psi(\kappa)=g \mid \delta_{f}$, we conclude that $\mu=\operatorname{ind}_{\mathfrak{R}_{\odot} \uparrow \mathfrak{R}} \nu$, where $\nu \in \hat{\mathfrak{I}}_{\sigma}$ and $\nu \mid 3$ is a multiple of $\sigma$. Hence, writing $F^{e}=\varphi\left(\mathfrak{R}_{\sigma}^{e}\right)$, and denoting by $\sigma^{e}$ a projective extension of $\sigma$ to $\mathfrak{R}_{\sigma}$, there is a $\nu^{\prime} \in \mathfrak{F}_{-1}^{e}$ such that $\nu=\left[\sigma^{e} \otimes\right.$ $(\nu \circ \varphi)]^{\sim}$. (2) We show next that $\mathfrak{F}^{e}$ commutes with $\Gamma_{0}$. In fact, if $a \in \mathfrak{F}^{e}$ and $l \in \gamma$, we have, by what we say in (b), proof of Lemma 10 , al $=1+c v$. Since, however, $\mathfrak{F}^{e}$ is an extension, by a subgroup of the center of $\Gamma_{0}$, of the finite group $\mathfrak{\Omega}_{o} / 3$, clearly $c=0$. Let us put $\Delta^{\prime}=\mathfrak{F}^{e} \cdot \Gamma_{0}$; by what we have just seen $\Delta^{\prime}$ is the direct product of $\mathfrak{F}^{e}$ with a vector group. Hence there is a $\bar{\nu}^{\prime} \in \hat{\Delta}^{\prime}$ such that $\bar{\nu}^{\prime} \mid \mathscr{F}^{e}=\nu^{\prime}$. This being so, let us set $\mathbb{\Re}^{\prime}=q\left(\bar{\varphi}^{-1}\left(\Delta^{\prime}\right)\right)$ and $K^{\prime}=h\left(\Phi\left(\Omega^{\prime}\right)\right)$; the latter is a closed subgroup, containing $N$, of $G_{\pi}$. Therefore, since $N$ is cocompact in $L, \bar{K}=K^{\prime} \cdot L$ is closed in $G$. In the following we shall show that $\bar{K}=\Pi$. Let us set $\tau^{\prime}=\left[\sigma^{e} \otimes\left(\bar{\nu}^{\prime} \circ \varphi\right)\right]^{\sim}$; we have $\tau^{\prime} \in{\hat{\aleph^{\prime}}}^{\prime}$ and $\tau^{\prime} \mid \mathfrak{I}_{0}=\nu$. Writing $\rho^{\prime}=\left[\pi^{e} \otimes(\tau \circ \Phi)\right]$, we form finally $\lambda^{\prime}=\operatorname{ind}_{K^{\prime} \uparrow \bar{K}} \rho^{\prime}$.

(c) We prove next that $\bar{K} \subseteq \Pi$. Taking into account the definition of $\Pi$, this will result from the following three statements: (1) $\lambda^{\prime} \mid L=\lambda$, (2) $\bar{K}$ is connected, (3) $\lambda^{\prime}$ is $\left(G_{\lambda}\right)_{0}$ invariant. Ad (1) Let us put $L_{\pi}^{\prime}=K^{\prime} \cap L$; we then have

$$
\lambda^{\prime} \mid L=\inf _{L_{*}^{\prime} \uparrow L}\left(\rho^{\prime} \mid L_{\pi}^{\prime}\right)=\operatorname{ind}_{L_{*} \uparrow L}\left(\operatorname{ind}_{L_{*}^{\prime} \uparrow L}\left(\rho^{\prime} \mid L_{\pi}^{\prime}\right)\right) .
$$

We observe that $\Re^{\prime} \cap \mathfrak{L}=\mathfrak{I}_{\sigma}$ implies $L_{\pi}^{\prime}=h\left(\Phi\left(\mathfrak{I}_{\sigma}\right)\right)$ and thus, by $\pi^{\prime} \mid \mathfrak{I}_{\sigma}=\nu$,

$$
\rho^{\prime} \mid L_{\pi}^{\prime}=\left[\pi^{e} \otimes(\nu \circ \Phi)\right]^{\sim} \text {. }
$$

Therefore, since $\mu=$ ind $_{\mathfrak{R}_{\ominus} \uparrow \mathfrak{Q}} \nu$, we obtain

$$
\operatorname{ind}_{L_{i}^{\prime} \uparrow L_{m}}\left(\rho^{\prime} \mid L_{\pi}^{\prime}\right)=\left[\pi^{e} \otimes(\mu \circ \Phi)\right]^{\sim}=\rho,
$$

and thus finally $\lambda^{\prime} \mid L=\operatorname{ind}_{L_{\uparrow} \uparrow L} \rho=\lambda$. Ad (2) We prove next that $\bar{K}$ is connected; to this end it is enough to show the same for $\bar{K} / L$. By what we saw in (1), we have $\bar{K} / L=K^{\prime} / L_{\pi}^{\prime}=\Omega^{\prime} / \Re_{0}$; thus the desired conclusion follows by observing that $\Omega^{\prime}=\Re_{0}^{\prime} \cdot \mathfrak{L}_{\sigma}$. Ad(3)( $\alpha$ ) Since $\lambda \mid N$ decomposes over $L \pi \subset \hat{N}$, we have, if $b \in G_{\lambda}, b \pi \in L \pi$, and thus $b \in G_{\pi} \cdot L$ and, hence, $G_{\lambda}=\left(G_{\lambda} \cap G_{\pi}\right) \cdot L$, and $\left(G_{\lambda}\right)_{0}=\left(G_{\lambda} \cap G_{\pi}\right)_{0} \cdot L$. Let $b$ be fixed in $\left(G_{\lambda} \cap\right.$ $\left.G_{\pi}\right)_{0}$; it will suffice to show that $b \lambda^{\prime}=\lambda^{\prime}$. $(\beta)$ We denote by the same letter also an element of $\mathfrak{M}$, which is the image of some element of $G_{\pi}^{e}$ over $b$. This being so we claim that $b \in\left(\mathfrak{M}_{\sigma}\right)_{0}$. We have, in fact, for any $b \in G_{\lambda} \cap G_{\pi}$ :

$$
\text { ind } b \rho=b \lambda=\lambda=\operatorname{ind}_{L_{\uparrow \uparrow L} \rho} \rho
$$

and thus, since $b \pi=\pi, b \rho=\rho$ also. This yields $b \mu=\mu$ and $b \sigma \in \mathfrak{L} \sigma$; 
therefore, if $b \in\left(G_{\lambda} \cap G_{\pi}\right)_{0}$, we conclude that $b \sigma=\sigma$, proving our statement. $(\gamma)$ We claim next that $b K^{\prime} b^{-1}=K^{\prime}$. To this end it is enough to note that by $\left[\mathfrak{M}_{\sigma}, \mathfrak{M}_{\sigma}\right] \subseteq \mathbb{N}^{\prime}$ the latter is invariant in $\mathfrak{M}_{\sigma}$. We infer from this that $b \lambda^{\prime}=$ ind $_{K^{\prime} \uparrow \bar{K}} b \rho^{\prime}$. Observing that $b \rho^{\prime}=\left[\pi^{e} \otimes\left(b \tau^{\prime} \circ \Phi\right)\right]^{\sim}$, to arrive at our goal it suffices to show that $b \tau^{\prime}=\tau^{\prime}$. But there is a $c \in \Gamma_{0}$ such that $b r^{\prime}=\left[\sigma^{e} \otimes\left(c \bar{\nu}^{\prime} \circ \varphi\right)\right]^{\sim}$ and thus the desired conclusion follows from the commutativity, established in (b) above, of $\Delta^{\prime}=\mathfrak{F}^{e} \cdot \Gamma_{0}$ and $\Gamma_{0}$.

(d) Next we complete the proof of our claim that $\Pi=\bar{K}$ (cf. (b) end) in the following manner. Assume that $\lambda^{\prime \prime} \in \Pi$ is invariant under $\left(G_{\lambda}\right)_{0}$ such that $\lambda^{\prime \prime} \mid L=\lambda$. (1) Since $\lambda^{\prime \prime}|N=\lambda| N$, putting $K^{\prime \prime}=\Pi_{n}$, there is a $\rho^{\prime \prime} \in \hat{K}^{\prime \prime}$ such that $\lambda^{\prime \prime}=\operatorname{ind}_{K^{\prime \prime} \uparrow \Pi} \rho^{\prime \prime}$ and $\rho^{\prime \prime} \mid N$ is a multiple of $\pi$. Evidently, we also have

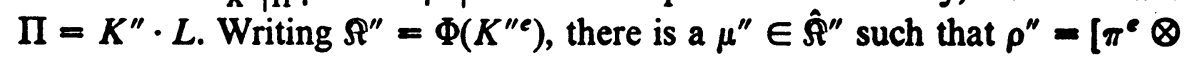
$\left.\left(\mu^{\prime \prime} \circ \Phi\right)\right]^{\sim}$. (2) Let us next observe that $\mu^{\prime \prime} \mid \mathbb{R}=\mu$. In fact, by $\Pi=K^{\prime \prime} \cdot L$ and $L_{\pi}=K^{\prime \prime} \cap L$ we have

$$
\operatorname{ind}_{L_{\pi} \uparrow L} \rho=\lambda=\lambda^{\prime \prime} \mid L=\operatorname{ind}_{L_{\varangle} \uparrow L}\left(\rho^{\prime \prime} \mid L_{\pi}\right)
$$

and hence

$$
\left[\pi^{e} \otimes(\mu \circ \Phi)\right]^{\sim}=\rho=\rho^{\prime \prime} \mid L_{\pi}=\left[\pi^{e} \otimes\left(\left(\mu^{\prime \prime} \mid \mathbb{R}\right) \circ \Phi\right)\right]^{\sim},
$$

and thus $\mu^{\prime \prime} \mid \mathfrak{R}=\mu$. With continued use of the notations of (b), we conclude from this that $\mu^{\prime \prime}=$ ind $_{\Omega_{n}^{\prime \prime} \uparrow \Omega^{\prime \prime}} \tau^{\prime \prime}$, where $\tau^{\prime \prime} \in \hat{\Omega}_{\sigma}^{\prime \prime}$ and $\tau^{\prime \prime} \mid \mathcal{B}$ is a multiple of 0 . Obviously $\Omega^{\prime \prime}=\Omega_{\alpha}^{\prime \prime} \cdot \mathfrak{R}$ also. Let us put $\Delta^{\prime \prime}=\varphi\left(\Omega_{\sigma}^{\prime \prime e}\right) \subset \Gamma$. There is a $\bar{\nu}^{\prime \prime} \in \hat{\Delta}^{\prime \prime}$ such that $\tau^{\prime \prime}=\left[\sigma^{\circ} \otimes\left(\bar{\nu}^{\prime \prime} \circ \varphi\right)\right]^{\sim}$. Evidently $\Delta^{\prime \prime} \supseteq \Delta^{\prime}$. To prove that $\Pi=\bar{K}$, it will be enough to show that $\Delta^{\prime \prime}=\Delta^{\prime}$ and $\bar{\nu}^{\prime \prime}=\bar{\nu}^{\prime}$. (2) Let us next observe that $\Delta^{\prime \prime} / \mathfrak{F}^{e}$ is connected. In fact, we have

$$
\Delta^{\prime \prime} / \mathfrak{F}^{e}=\Omega_{\sigma}^{\prime \prime} / \mathfrak{R}_{\sigma}=\mathfrak{\Omega}^{\prime \prime} / \mathfrak{R}=K^{\prime \prime} / L_{\pi}=\Pi / L
$$

and thus it suffices to recall that $\Pi$ is connected. Hence, in particular, we can conclude that $\Delta^{\prime \prime}=\mathfrak{F}^{e} \cdot\left(\Delta^{\prime \prime}\right)_{0}$. (3) We remark that $\bar{\nu}^{\prime \prime} \mid \mathfrak{F}^{e}=\nu^{\prime}$. In fact, since $\mathfrak{S}^{\prime \prime}=\mathfrak{R}_{0}^{\prime \prime} \cdot \mathfrak{L}, \mathfrak{I}_{0}=\mathfrak{S}_{0}^{\prime \prime} \cap \mathfrak{L}$ and $\mu^{\prime \prime} \mid \mathfrak{L}=\mu$ (cf. (1) above) we have

$$
\text { ind } \nu=\mu=\mu^{\prime \prime} \mid \mathfrak{R}=\operatorname{ind}_{\mathfrak{S}_{0} \uparrow \mathfrak{R}}\left(\tau^{\prime \prime} \mid \mathfrak{R}_{\mathrm{o}}\right) \text {, }
$$

and hence $\tau^{\prime \prime} \mid \mathfrak{R}_{\sigma}=\nu$ and $\bar{\nu}^{\prime \prime} \mid \mathfrak{F}^{e}=\nu^{\prime}$. (4) By what we saw in (2), $\Delta^{\prime \prime}$ is normalized by $\Gamma_{0}$. This being so, we next show that $\bar{\nu}^{\prime \prime} \in \hat{\Delta}^{\prime \prime}$ is invariant with respect to $\Gamma_{0}$. ( $\left.\alpha\right)$ Putting $H=\left(G_{\lambda} \cap G_{\pi}\right)_{0}$ we claim that $\left(\mathbb{R}_{0}\right)_{0}=\Phi\left(H^{e}\right)$. We already know from (c), $\operatorname{Ad}(3),(\beta)$, that $\Phi\left(H^{e}\right) \subseteq\left(\mathbb{R}_{o}\right)_{0}$. With notations as loc. cit., let us assume that $b \in\left(\mathbb{R}_{o}\right)_{0}$; it is enough to prove that $b \lambda=\lambda$. Since $\nu \in \hat{\mathfrak{L}}_{\sigma}$ and $\nu \mid 3=\sigma$, we clearly have $b \nu=\nu$, and thus $b \mu=\mu, b \rho=\rho$ and finally $b \lambda=\lambda$. $(\beta)$ To complete our reasoning, we make use of the invariance, with respect to $\left(G_{\lambda}\right)_{o}$, of $\lambda^{\prime \prime}$ in the following manner. Assume that $b \in H$. Since $\lambda^{\prime \prime}=\operatorname{ind}_{K^{\prime \prime} \uparrow \Pi} \rho^{\prime \prime}$, we have $b \rho^{\prime \prime}=\rho^{\prime \prime}$, and hence $b \mu^{\prime \prime}=\mu^{\prime \prime}$ and $b \tau^{\prime \prime}=\tau^{\prime \prime}$. Since $\tau^{\prime \prime}=\left[\sigma^{e} \otimes\left(\bar{\nu}^{\prime \prime} \circ \varphi\right)\right]^{\sim}$ and $\Gamma_{0}=\varphi\left(\left[\left(\mathbb{M}_{\sigma}\right)_{0}\right]^{e}\right)$, by what we saw in $(\alpha)$ we 
conclude that $\bar{\nu}^{\prime \prime}$ is $\Gamma_{0}$ invariant. (5) We prove finally that $\left(\Delta^{\prime \prime}\right)_{0} \subseteq \Gamma_{0}^{4}$; whence, by (2), $\Delta^{\prime \prime} \subseteq \Delta^{\prime}$ and thus $\Pi=\bar{K}$. Since $\bar{\nu}^{\prime \prime}\left|\mathscr{F}^{e}=\nu^{\prime} \in \hat{\mho}^{e}, \bar{\nu}^{\prime \prime}\right|\left(\Delta^{\prime \prime}\right)_{0}$ is a multiple of a $\Gamma_{0}$ invariant character $\varphi$ of $\left(\Delta^{\prime \prime}\right)_{0}$. If $\gamma \in \Gamma_{0}$ and $\delta \in\left(\Delta^{\prime \prime}\right)_{0}$ we have $\gamma \delta \gamma^{-1}=u \delta(u \in T)$, whence

$$
\varphi(\delta)=\varphi\left(\gamma \delta \gamma^{-1}\right)=\varphi(u) \varphi(\delta)
$$

and thus $\varphi(u)=1$. Since $\nu^{\prime} \in \mathfrak{F}_{-1}^{e}$, this implies at once that $u=e$ and therefore $\left(\Delta^{\prime \prime}\right)_{0} \subseteq \Gamma_{0}^{\natural}$, and $\Pi=K^{\prime} \cdot L$.

(e) Below we use again the following observations. If $\mathfrak{D}$ is a closed subgroup of $\mathfrak{M}$, such that $D \supseteq \Psi(\mathscr{D})$, then $h\left(\Phi^{-1}(\mathfrak{D})\right)=p\left(\Psi^{-1}(\mathfrak{D})\right) \cdot N$. Similarly, if $\Delta$ is closed in $\Gamma$ such that $\Delta \supseteq T$, then $q\left(\varphi^{-1}(\Delta)\right)={ }^{-1}(\Delta) \cdot 3$. This being so, to complete the proof of our lemma we recall that by what we saw above we have

$$
K^{\prime}=h\left(\Phi^{-1}\left(\Omega^{\prime}\right)\right), \quad{\mathbb{\Omega ^ { \prime }}}^{\prime}={\Omega_{0}^{\prime}}_{0} \cdot \mathfrak{\Omega}_{\sigma}, \quad \Omega_{0}^{\prime}=q\left({ }_{\varphi}^{-1}\left(\Gamma_{0}^{\dot{q}}\right)\right)=\psi^{-1}\left(\Gamma_{0}^{q}\right) \cdot 3 .
$$

Also, by Lemma 10 , setting $\Lambda=\psi \circ\left(\Psi \mid \mathcal{S}^{\prime}\right)$ we obtain $p\left(\Lambda^{-1}\left(\Gamma_{0}^{\xi}\right)\right)=$ $\left(G_{g}\right)_{0}\left(L_{h}\right)_{0} N_{f}$. Hence, by the final conclusion of (d) above,

$$
\Pi=K^{\prime} \cdot L=p\left({ }^{-1}\left(\Omega_{0}^{\prime}\right)\right) \cdot L=p\left(\Lambda^{-1}\left(\Gamma_{0}^{\xi}\right)\right) L=\left(G_{g}\right)_{0} L,
$$

which is the desired result. Q.E.D.

In the following we continue to use the notations of Lemma 11; in particular, $\lambda$ stands for a fixed element of $\hat{L}$, and $\lambda^{\prime} \in \hat{\Pi}$ satisfies $\lambda^{\prime} \mid L=\lambda$. We write $\Pi=\exp \left(\mathfrak{f}^{\prime}\right)$ (A.4) and observe that if $g \in g^{\prime}$ is such that $g \mid \delta=h \in$ $\eta(\lambda)$ then by Lemma $11 \mathfrak{f}=\mathfrak{d}+g_{g}$. Let $\Sigma$ be the orthogonal complement of $d$ in $\eta^{\prime}$. Given $v \in \Sigma$ we write $\varphi_{v}$ for the element of $Y(\Pi / L)($ A.5) determined by $d \varphi_{\nu}=i v$. In the following \& will stand for some fixed connected Lie group containing $G$ as an invariant subgroup.

LEMMA 12. With the above notations let us put $\mathbb{S}_{h}^{\prime}=\mathbb{B}_{h} \cap \mathbb{S}_{\lambda}, e=g \mid \mathbb{t}$ and, for some $a \in \mathbb{B S}_{h}^{\prime}, a e=e+v(v \in \Sigma)$. Then we have $a \lambda^{\prime}=\varphi_{v} \lambda^{\prime}$.

Proof. If the kernel of $\lambda$ contains $N$, by what we saw in (a), proof of Lemma 11, we have $\Pi=G$ and hence also $g=e$. We note next that if $r$ is the radical of $\mathfrak{g}, \&$ acts trivially on $\mathrm{r} / \mathfrak{n}$. Hence $a \in \mathbb{B}$ implies $a \lambda^{\prime}=\lambda^{\prime}$. By the same token, if $a \in \mathbb{B}_{h}$, we also have $a g=g$, proving the validity of our claim in this special case. In the following we shall assume, that $\lambda \mid N \not I$; then (cf. (a), proof of Lemma 6) $g \mid \mathfrak{n}=f \neq 0$.

(a)(1) We start by observing that, since $\mathbb{S}_{h} \subseteq \mathbb{S}_{f} \subseteq \mathbb{S}_{f} N=\mathbb{B}_{\pi}, \mathbb{S}_{h}$ operates on $G_{\pi}^{e}$ and evidently also on $\mathfrak{D}=G_{\pi}^{e} / N$. We put, as in $2.4, \mathcal{L}={ }^{-1}\left(L_{f}\right) \subset \mathcal{G}$, 
$\Psi^{\prime}=\Psi \mid \mathcal{L}$ and recall (cf. the diagram of 3.1) that we are assuming $\delta(\sigma)=3 \kappa$, where $\delta \Psi^{\prime}(\kappa)=h \mid \delta_{f}$. We infer from this, by virtue of $1.1(e)$, that if $a \in \mathbb{S}_{h}$, then $a \kappa=\kappa$ and hence, by Lemma 4, $a \sigma=\sigma$. This implies that $\mathbb{S}_{h}$ operates on $\mathfrak{R}_{\kappa}$ and $\mathfrak{R}_{\sigma}$, and thus also on $\mathfrak{M}_{\sigma}^{e}$ and $\Gamma=\mathfrak{R}_{\sigma}^{e} / 3$. For later use we recall (cf. 2.3, Remark 2) that the actions on $\Gamma$, derived from those on $\mathfrak{R}_{o}$ and $\mathfrak{M}_{\kappa} / \dot{\mathfrak{Z}}_{k}$, respectively, are identical. (2) We next observe that $\mathbb{S}_{h}$ normalizes $K^{\prime}$. Since $G_{g}$ depends on $g \mid d$ only, to this end it is enough to establish that $K^{\prime}=\left(G_{g}\right)_{0} L_{h}\left(L_{f}\right)_{0} N$. This follows easily from parts of Lemma 11 (cf., in particular, (e) loc. cit.) along with Lemma 10. In fact, it suffices to recall that

$$
\begin{gathered}
\mathfrak{R}^{\prime}=\mathfrak{L}_{0} \mathbb{\Re}_{0}^{\prime}=\mathfrak{R}_{\kappa} \stackrel{-1}{\psi}^{-1}\left(\Gamma_{0}\right) \mathfrak{3}, \\
K^{\prime}=p\left(\stackrel{-1}{\Psi}\left(K^{\prime}\right)\right) N \text { and } p\left(\stackrel{-1}{\Psi}\left(\mathfrak{I}_{\kappa}\right)\right)=L_{h} N_{f}
\end{gathered}
$$

(replace in Lemma 8, $G$ through $L$ ). This implies at once that $\mathbb{S}_{h}$ normalizes ${\Omega^{\prime}}^{\prime}$ and thus also $\Delta^{\prime}(3)$ Let us suppose that $\bar{\nu}^{\prime} \in \hat{\Delta}^{\prime}$ corresponds, in the sense of (b), proof of Lemma 11, to $\lambda^{\prime} \in \hat{\Pi}$. We are going to show that, upon replacing $\lambda^{\prime}$ by $a \lambda^{\prime}\left(a \in \mathbb{S}_{h}\right), \bar{\nu}^{\prime}$ gets replaced by $a \bar{\nu}^{\prime}$. In fact, we note first that, by (2), $a \lambda^{\prime}=\operatorname{ind}_{K^{\prime} \uparrow \bar{K}} a \rho^{\prime}$ and that $a \rho^{\prime}=\left[\pi^{e} \otimes\left(a \tau^{\prime} \circ \Phi\right)\right]^{-}$. Since $a \sigma=$ $\sigma$, this indeed implies that $a \tau^{\prime}=\left[\sigma^{e} \otimes\left(a \bar{\nu}^{\prime} \circ \varphi\right)\right]^{-}$. (4) Let us next observe that $a \in \mathbb{B S}_{h}^{\prime}=\mathbb{B}_{\lambda} \cap \mathbb{S}_{h}$ yields $a \nu^{\prime}=\nu^{\prime}$. In fact, if $a \in \mathbb{S}_{\pi} \cap \mathbb{S}_{\lambda}$, we have

$$
\text { ind } \rho=\lambda=a \lambda=\operatorname{ind}_{L_{\pi} \uparrow L} a \rho,
$$

and thus also $a \rho=\rho$ and $a \mu=\mu$. Therefore, by $a \sigma=\sigma, a \nu=\nu$ and $a \nu^{\prime}=\nu^{\prime}$. (5) If $\chi \in X\left(\Gamma_{0}^{4}\right)$ is such that $d \chi=i \alpha\left(\alpha \in\left(\gamma^{\natural}\right)^{\prime}\right)$, we write $\chi=\chi_{\alpha}$. If $\bar{\nu}^{\prime} \mid \Gamma_{0}^{\natural}$ is a multiple of $\chi_{\eta}$, by $a \nu^{\prime}=\nu^{\prime}$ we have $a \eta=\eta+w$ with $(v, w)=0$, where we again put $T=\exp (\mathrm{R} v)$. Defining $\chi_{w}^{\prime} \in X\left(\Delta^{\prime}\right)$ by $\chi_{w}^{\prime} \mid \mathcal{F}^{e} \equiv 1$ and $\chi_{w}^{\prime} \mid \Gamma_{0} \equiv \chi_{w}$, using (4) we conclude that $a \bar{\nu}^{\prime}=\chi_{w}^{\prime} \bar{\nu}^{\prime}\left(a \in \mathfrak{S}_{h}^{\prime}\right)$.

(b) In the following if a capital script $\left(\mathcal{E}\right.$, say) stands for a subgroup of $G_{f}$, we shall put $\mathcal{E}^{\prime}={ }_{p}^{-1}(\mathcal{E})$. Also if $\varphi \in X(\mathcal{E})$, we shall write $\varphi^{\prime}=\varphi \cdot\left(p \mid \mathcal{E}^{\prime}\right)$ $\in X\left(\mathcal{E}^{\prime}\right)$. Setting $\mathcal{C}=p\left(\Lambda^{-1}\left(\Gamma_{0}^{\dagger}\right)\right)$ we claim that there is an isomorphism $j$ from $Y(\Pi / L)$ onto $Y\left(\Delta^{\prime} / \mathfrak{F}^{e}\right)$ such that $(\alpha)$ for $\varphi \in Y(\Pi / L)$, the substitution $\lambda^{\prime} \mapsto \varphi \lambda^{\prime}$ is equivalent to $\bar{\nu}^{\prime} \mapsto j(\varphi) \bar{\nu}^{\prime} ;(\beta) j(\varphi) \circ\left(\Lambda \mid \mathcal{C}^{\prime}\right)=(\varphi \mid \mathcal{C})^{\prime}$. In fact, (A) we write $\mathcal{Q}=p\left(\bar{\psi}^{-1}\left(\Omega^{\prime}\right)\right)$ and show the existence of an isomorphism $i: Y(\Pi / L)$ $\rightarrow Y\left(\Omega^{\prime} / \Omega_{\sigma}\right)$ such that $(\alpha)$ for $\varphi \in Y(\Pi / L), \lambda^{\prime} \mapsto \varphi \lambda^{\prime}$ is equivalent to $\tau^{\prime} \mapsto i(\varphi) \tau^{\prime} ;(\beta) i(\varphi) \circ\left(\Psi \mid \mathcal{Q}^{\prime}\right)=(\varphi \mid \mathcal{Q})^{\prime}$. In fact, putting $\psi=\varphi \mid K^{\prime}$ we first have $\varphi \lambda^{\prime}=$ ind $_{K^{\prime} \uparrow \Pi} \psi \rho^{\prime}$. If we write $\psi^{e}=\psi \circ\left(h \mid K^{\prime e}\right) \in X\left(K^{\prime e}\right)$, there is a $\zeta \in X\left(K^{\prime}\right)$ with

$$
\zeta \circ\left(\Phi \mid K^{\prime e}\right)=\psi^{e} \text { and } \psi \rho^{\prime}=\left[\pi^{e} \otimes\left(\zeta \tau^{\prime} \circ \Phi\right)\right]^{\sim} .
$$

We next show that $\zeta \circ\left(\Psi \mid \mathbb{Q}^{\prime}\right)=(\varphi \mid \mathbb{Q})^{\prime}$. In fact, to this end it is enough to 
prove that $a^{\prime} \in \mathbb{Q}^{\prime}$ and $p\left(a^{\prime}\right)=a$ implies $\zeta\left(\Psi\left(a^{\prime}\right)\right)=\varphi(a)$. But by the diagram of 1.1(d), $\zeta\left(\Psi\left(a^{\prime}\right)\right)=\psi^{e}\left(\gamma\left(a^{\prime}\right)\right)$ which, since $h\left(\gamma\left(a^{\prime}\right)\right)=a$, is equal to $\varphi(a)$. Since $K^{\prime}=\mathbb{Q} N$, we have $\Pi=K^{\prime} \cdot L=\mathbb{Q} L$, and hence the map $\varphi \mapsto \zeta$ is an injection. To conclude that it is an isomorphism, it suffices to note that $p\left(\Psi\left(\mathfrak{Q}_{\odot}\right)\right)=\mathbb{Q} \cap L$, which relation follows from

$$
\mathfrak{R}^{\prime} \cap \mathfrak{L}=\mathfrak{L}_{0} \text { and } \mathbb{Q} \cap L=\mathbb{Q} \cap L_{f}=p\left(\Psi^{-1}\left(\mathscr{I}^{\prime} \cap \mathfrak{R}\right)\right) \text {. }
$$

(B) We write $\mathfrak{A}=\psi^{-1}\left(\Delta^{\prime}\right)$ and observe that $\mathfrak{A} \cap \mathfrak{L}=\psi\left(\mathfrak{F}^{e}\right)$. Imitating the previous reasoning one shows easily that there is an isomorphism from $Y\left(\Omega^{\prime} / \mathfrak{R}_{\sigma}\right)$ onto $Y\left(\Delta^{\prime} / \mathscr{F}^{e}\right)$ such that $(\alpha)$ for $\zeta \in Y\left(\Omega^{\prime} / \mathfrak{\Omega}_{\sigma}\right)$ the substitution $\tau^{\prime} \mapsto \zeta \tau^{\prime}$ is equivalent to $\bar{\nu}^{\prime} \mapsto i(\zeta) \bar{\nu}^{\prime} ;(\beta) i(\zeta) \circ(\psi \mid \mathfrak{2})=\zeta \mid \mathcal{A}$. This being so, let $i_{1}$ and $i_{2}$ be as $i$ in (A) and (B) respectively. Setting $j=i_{2} \circ i_{1}$, it is clear that $j$ is an isomorphism from $Y(\Pi / L)$ onto $Y\left(\Delta^{\prime} / \mathfrak{F}^{e}\right)$ having the properties claimed at the start of $(b)$.

(c) Let us put $\mathcal{C}=\exp (\mathfrak{c})\left(\mathfrak{c}=\mathfrak{g}_{g}+\mathfrak{d}_{h}+\mathfrak{n}_{f}\right)$ and $\Lambda^{\prime}=\Lambda \mid \mathcal{C}$. If $a \in \mathbb{S}_{h}^{\prime}$, by (a)(5) and (b) we can conclude that $a \lambda^{\prime}=\varphi \lambda^{\prime}$, where $\varphi \in Y(\Pi / L)$ is determined by $\chi_{w} \circ \Lambda^{\prime}=(\varphi \mid \mathcal{C})^{\prime}$. Putting $\varphi=\varphi_{z}(z \in \Sigma)$ we infer that $\delta \Lambda^{\prime}(w)$ $=z \mid c$. To complete the proof of our lemma we have to show that $z=v$, where $v$ is determined by $a e=e+v$. Evidently, we can assume $\delta \Lambda^{\prime}(\eta)=$ $g \mid c$. Therefore we have

$$
g|c+v| c=a g\left|c=\delta \Lambda^{\prime}(a \eta)=\delta \Lambda^{\prime}(\eta)+\delta \Lambda^{\prime}(w)=g\right| c+z \mid c,
$$

whence $v|\mathfrak{c}=z| c$. Since, however, $\mathfrak{f}=\mathfrak{d}+\mathfrak{c}$, this implies $v=z$. Q.E.D.

3.3. The proof of the following lemma is very similar to that of Lemma 2.

Lemma 13. Assume that $\mathfrak{m}$ is a subspace of $\mathfrak{g}$ containing $[\mathfrak{g}, \mathfrak{g}]$. Given $g \in \mathfrak{g}^{\prime}$ we set $\mathfrak{f}=\mathfrak{m}+g_{g}$ and $h=g \mid \mathfrak{m}$. Then we have $\left(G_{h}\right)_{0} g=g+\mathfrak{f}^{\perp}$.

Proof. (a) Let us suppose that $\left\{l_{j} ; 1<j<n\right\} \subset g_{h}$. We claim that if $n>2$, then $l_{1} \cdot l_{2} \cdots l_{n} g=0$. In fact, if $v$ denotes the left-hand side and $l \in \mathfrak{g}$, we have $(l, v)=\left(\left[l_{n}, u\right], g\right)$, where $u \in[g, g] \subset m$. Thus, by $l_{n} \in g_{h}$ and $h=g \mid \mathfrak{n},(l, v)=0$ and hence, since $l \in \mathfrak{g}$ is arbitrary, $v=0$. Observe that $g_{h} g$ is evidently orthogonal to $f$. (b) To complete our proof it will be enough to show that $\operatorname{dim}\left(\mathfrak{f}^{\perp}\right)=\operatorname{dim}\left(g_{h} \cdot g\right)$. But to this end it suffices to note that

$$
\begin{aligned}
\operatorname{dim}\left(\mathfrak{g}_{h} \cdot \mathfrak{g}\right) & =\operatorname{dim}\left(\mathfrak{g}_{h}\right)-\operatorname{dim}\left(\mathfrak{g}_{\mathfrak{g}}\right)=\operatorname{dim}(\mathfrak{g})-\operatorname{dim}(\mathfrak{m})+\operatorname{dim}\left(\mathfrak{m}_{\mathfrak{g}}\right)-\operatorname{dim}\left(\mathfrak{g}_{\mathfrak{g}}\right) \\
& =\operatorname{dim}(\mathfrak{g})-\operatorname{dim}\left(\mathfrak{m}+\mathfrak{g}_{\mathfrak{g}}\right)=\operatorname{dim}\left(\mathfrak{f}^{\perp}\right) . \text { Q.E.D. }
\end{aligned}
$$

3.4. Before stating Lemma 14, which summarizes the results of this section, we make a series of observations. (1) In the following we shall take $B 5=$ $\exp (\tilde{\mathfrak{g}})(\mathrm{A} .4)$, where $\tilde{\mathfrak{g}}$ contains $\mathfrak{g}$ such that $[\tilde{\mathfrak{g}}, \tilde{g}]=[\mathfrak{g}, \mathfrak{g}]$ and is isomorphic to an algebraic Lie algebra of endomorphisms on a finite dimensional real vector space (cf. $[20,1.1$, p. 84]). To obtain $\tilde{\mathfrak{g}}$ as just indicated, we may 
proceed as follows. First, by the theorem of Ado, we can assume that $g$ is isomorphic to a Lie algebra of endomorphisms. Next, let us denote by $\mathfrak{r}$ the radical of $\mathfrak{g}$ and by $\mathfrak{h}$ a Levi section. If $\tilde{r}$ is the smallest algebraic Lie algebra of endomorphisms containing $\mathfrak{r}$, we can take $\mathfrak{g}=\mathfrak{h}+\tilde{\mathfrak{r}}$. Note, in particular, that we may assume that just as for $G$, the radical of \& is cocompact. Observe also that $G$ acts trivially on $\mathfrak{d}^{\perp} \subset \mathfrak{g}^{\prime}$. (2) Let $\operatorname{Prim}(G)$ be the set of all primitive ideals of $C^{*}(G)$. We recall (cf. [20, Lemma 1.1.8, p. 89]) that if $J \in \operatorname{Prim}(G)$, there is a well-determined \&S orbit $E(J)$ on $\hat{L}$ with the following property: If $T$ is a factor representation of $G$, such that the kernel $\operatorname{ker}(T)$ of the associated * representation of $C^{*}(G)$ is equal to $J$, then the direct integral decomposition of $T \mid L$ over $\hat{L}$ is carried by $E(J)$. (3) Let us fix $\lambda$ in $\hat{L}$. For some $\rho \in \Pi$ (ח being as in Lemma 12) satisfying $\rho \mid L=\lambda$, let us put $\mathfrak{U}=G_{\rho}$; then $\mathfrak{U}$ depends on $\lambda$ only. This being so, we recall the following result (cf. [18, Lemma 3.5, p.484]). Let $\mathscr{B}$ be the family of all those closed subgroups containing $L$ of $G$ to which $\lambda$ admits a trivial $\mathfrak{U}$ invariant extension. Then $\mathscr{G}$ admits a well-determined maximal element $K$ and $K_{0}=\Pi$. We also note (cf. [20, Lemma 1.1.4, p. 86]) that $K, \Pi, \mathfrak{u}$ do not change if $\lambda$ is confined to the same orbit $E \in \hat{L} / \mathbb{S}$. (4) $E$ being the same, let us put $F=\{\tau ; \tau \in \hat{K}, \tau \mid L \in E\}$. Then $F$, as a subspace of $\hat{K}$, is locally compact, separable and Hausdorff (cf. [20, Lemma 1.1.5, p. 87]; note that by the proof loc. cit. this is always so if \& $/ L$ is abelian). If $E=E(J)$ we shall sometimes write $F=F(J)$. (5) We recall (cf. [20, pp. 110-111]) that we call a primitive ideal $J$ of type $I$ if for an irreducible representation $T$, with $\operatorname{ker}(T)=J$, $T\left(C^{*}(G)\right)$ contains a nonzero compact operator. $J$ is of type $I$ if and only if (A) $F(J) / G$ is a $T_{1}$ space, (B) $n(J)<+\infty$, where $n(J)$ is the order $(<+\infty)$ of the countable group $\mathfrak{u} / K\left(G_{\lambda}\right)_{0}$ formed with $E=E(J)$ (cf. [20, Proposition 3, p. 111]).

3.5. Below $\eta$ will have the same meaning as in Lemma 6 .

Lemma 14. Let $J$ be some element in $\operatorname{Prim}(G)$. With the previous notations, we write $\Omega(J)$ for the complete inverse image in $g^{\prime}$ of $\eta(E(J)) \subset \mathfrak{D}^{\prime} / L$. Then $F(J) / G$ is a $T_{1}$ space if and only if so is $\Omega(J) / G$.

Proof. (a) Let us select some $\lambda \in E(J)$ and $h \in \eta(\lambda)$; these are to be kept fixed in the sequel. \&S being as in 3.4(1), we put, as in Lemma 12, $\mathbb{S}_{h}^{\prime}=\mathbb{S}_{\lambda} \cap$ $\mathbb{S}_{h}$, and claim that $\mathbb{S}_{\lambda}=\mathbb{S}_{h}^{\prime} \cdot L$. To this end it is enough to show that $\mathbb{B}_{\lambda} \subseteq \mathbb{S}_{h} L$. But if $a \in \mathbb{S}_{\lambda}$, we have by Lemma 6:

$$
a L h=a \eta(\lambda)=\eta(a \lambda)=\eta(\lambda)=L h,
$$

and hence $a h \in L h$ and $a \in \mathbb{B}_{h} \cdot L$.

(b) Supposing that $g \in g^{\prime}$ is such that $g \mid \delta=h$, we again write $f=\delta+g_{g}$, $e=g \mid \mathfrak{t}$ and denote by $\Sigma$ the orthogonal complement of $\delta$ in $\mathfrak{f}^{\prime}$. Let $\tilde{S}$ be the direct product $\& \times \Sigma$; it acts on $\mathfrak{l}^{\prime}$ by $(a, v) d=a d+v\left(d \in \mathfrak{f}^{\prime} ;\right.$ cf. $\left.3.4(1)\right)$. 
Let $j$ be the restriction to $\tilde{\Theta}_{e}$ of the canonical projection $\tilde{\Im} \rightarrow \circlearrowleft$. One verifies at once that $j$ gives rise to an isomorphism between $\tilde{G}_{e}$ and $\mathbb{S}_{h}$. Putting $\tilde{\Theta}_{e}^{\prime \prime}=j\left(\mathscr{E}_{h}^{\prime}\right)$ we claim that $\tilde{\Theta}_{e} / \tilde{\Theta}_{e}^{\prime}$ is finite. To show this it suffices to establish the analogous fact for $\mathbb{S}_{h} / \mathbb{S}_{h}^{\prime}$. With the notations of (a), proof of Lemma 12 , let us note that, $\mathfrak{L}=\Phi\left(L_{\pi}^{e}\right)$ being compact, the set $\left\{\tau ; \tau \in \hat{\mathfrak{\Omega}}_{o}\right.$, $\tau \mid 3=\sigma\}$ is finite. On the other hand (cf. (a)(4) loc. cit.), if $a \in \mathbb{S}_{h}$, the substitution $\lambda \mapsto a \lambda$ is equivalent to $\nu \mapsto a \nu\left(\in \hat{\mathfrak{E}}_{o}\right)$ and, in particular, we have $\lambda=a \lambda$ if and only if $\nu=a \nu$. Thus, since $\mathscr{S}_{h}^{\prime}=\mathbb{S}_{h} \cap \mathbb{S}_{\lambda}, \mathbb{S}_{h} / \mathbb{S}_{h}^{\prime}$ is finite.

(c) Let us define an action of ङङ on $\Pi$ by $(a, v) \tau=\varphi_{v} a \tau$, where $\varphi_{v} \in X(\Pi)$ is again determined by $d \varphi_{v}=i v$. Let $\rho$ be a fixed element of $\hat{\Pi}$ such that $\rho \mid L=\lambda$. We claim that $\tilde{S}_{\rho}=\tilde{G}_{e}^{\prime} L$. In fact, $(1)$ if $(a, v) \in \tilde{G}_{e}^{\prime}$, we have $a \in \mathbb{S}_{h}^{\prime}$ and $a e=e-v$. Therefore replacing $\lambda^{\prime}$ by $\rho$ in Lemma 12, we conclude that $a \rho=\varphi_{-v} \rho$. Hence $(a, v) \in \tilde{G}_{\rho}$ and thus $\tilde{G}_{e}^{\prime} L \subseteq \tilde{E}_{\rho}$. (2) To establish the opposite inclusion let us observe that if $(a, v) \in \tilde{\mathbb{E}}_{\rho}$, then $\varphi_{v} a \rho=\rho$ and thus $a \lambda=\lambda$ and $a \in \mathbb{S}_{\lambda}=\mathscr{G}_{h}^{\prime} \cdot L$ (cf. (a)). In this manner we can assume from the start that $a \in \mathcal{O}_{h}^{\prime}$. Putting $a e=e+w$ we have by Lemma 12: $\varphi_{w} \rho=a \rho=$ $\varphi_{-0} \cdot \rho$, whence $w=-v$ and $(a, v) \in \tilde{G}_{e}^{\prime \prime}$, completing the proof of our statement.

(d) Let us set

$$
F_{0}=\{\tau ; \tau \in \hat{\Pi}, \tau \mid L \in E(J)\} .
$$

Replacing $G$ by $\Pi$ in 3.4(4), we conclude at once that $F_{0}$ as a subspace of $\hat{\Pi}$ is locally compact, separable and Hausdorff. To simplify notations, in the following we shall write $\Omega$ in place of $\Omega(J)$. Setting $\Omega^{\prime}=\Omega \mid \mathfrak{f} \subset \mathfrak{f}^{\prime}$, we are going to show that $F_{0} / G$ is a $T_{1}$ space if and only if so is $\Omega^{\prime} / G$. To this end

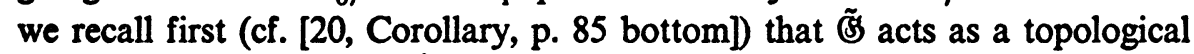
transformation group on $\hat{\Pi}$. Since $F_{0}=\tilde{\Theta} \rho$ we conclude that the map from $\tilde{\mathbb{S}} / \tilde{G}_{\rho}$ onto $F_{0}$, which sends $a \tilde{\mathbb{S}} \rho$ into $a \rho$, is a homeomorphism. Hence $F_{0} / G$ is a $T_{1}$ space if and only if $\tilde{S}_{\rho} \cdot G$ is closed. We have by (c), $\tilde{\Theta}_{\rho} G=\tilde{G}_{e}^{\prime \prime} \cdot G$, and the right-hand side, by (b), is cofinite in $\tilde{G}_{e} \cdot G$. Thus the latter is closed if and only if $F_{0} / G$ is a $T_{1}$ space. This being so, since $\Omega^{\prime}=\tilde{\Theta} e$, we shall have completed the proof of our starting claim by showing that $\Omega^{\prime} \subset \mathfrak{l}^{\prime}$ is locally closed. By virtue of our choice of \&S (cf. 3.4(1)), it acts on ' $b^{\prime}$ as the connected component of the identity of an algebraic subgroup of $G L\left(\delta^{\prime}\right)$. Hence $S^{S} h$ is locally closed in ' $\mathfrak{b}^{\prime}$ (cf. e.g. [19, p. 379] and the references loc. cit.) and thus the same holds true for its complete inverse image $\Omega^{\prime}$ in $\mathfrak{t}^{\prime}$.

(e) Since, by Lemma 13, any $G$ orbit $O$ in $\Omega$ is invariant under translations by $\mathcal{f}^{\perp} \subset \mathfrak{g}^{\prime}$, we conclude that $\Omega / G$ is a $T_{1}$ space if and only if $F_{0} / G$ is.

(f) We shall complete the proof of Lemma 14 by showing that if one of the spaces $F_{0} / G$ or $F(J) / G$ is $T_{1}$, then so is the other. Let us form the direct 
product $\mathfrak{A}=G \times Y(K / L)$. It acts as a topological transformation group on $K$ by the rule $(a, \psi) \sigma=\psi a \sigma((a, \psi) \in \mathfrak{A} ; \sigma \in \hat{K}$; cf. (d) above). Let $\tau \in \hat{K}$ be fixed such that $\tau \mid \Pi=\rho$. We have $F(J)=\mathfrak{A} \tau$ and, since $F(J)$ is locally compact and Hausdorff (cf. 3.4(4)), the map $\mathfrak{A} \ni \mathfrak{a} \mapsto$ a gives rise to an $\mathfrak{A}$ equivariant homeomorphism between the connected abelian Lie group $\mathfrak{A}=$ $\mathfrak{A} / \mathfrak{A}_{\tau}$ and $F(J)$. A similar construction, starting with $\mathfrak{U}_{1}=G \times Y(\Pi / L)$, leads to an analogous identification between $\mathfrak{U}_{1}=\mathfrak{A}_{1} /\left(\mathfrak{U}_{1}\right)_{p}$ and $F_{0}$. We denote by $p$ the morphism $A \rightarrow A_{1}$ resulting from the morphism $\mathfrak{A} \rightarrow \mathfrak{A}_{1}$ which corresponds to restriction to $\Pi$ of elements of $Y(K / L)$. The kernel of $p$ is isomorphic to the dual of the free abelian group $K / \Pi$. Let $A^{\prime}$ be the image of $G \tau$ in $A$. Then $F(J) / G\left(F_{0} / G\right)$ is a $T_{1}$ space if and only if $A^{\prime}$ (resp. $\left.p\left(A^{\prime}\right)\right)$ is closed. Let us next observe that $A^{\prime} \cap \operatorname{ker}(p)=\{e\}$. To this end it is enough to note that since $\tau$ is $\mathfrak{U}$ invariant (cf. 3.4(3)) we have $G_{\tau}=G_{\rho}=\mathfrak{u}$. In this manner, to complete our proof it suffices to invoke the following elementary statement. Let $B$ be a connected abelian Lie group, $T$ a closed subgroup, $B^{\prime}$ a connected subgroup such that $T \cdot B^{\prime}=B$ and $T \cap B^{\prime}=\{e\}$. Then $B^{\prime}$ is closed in $B$. Q.E.D.

3.6. Let us put $Z=\delta^{\perp} \subset \mathfrak{g}^{\prime}$ and define $P$ as the direct product of $B$ and of Z. $P$ acts on $g^{\prime}$ by the rule $(a, v) g=a g+v\left((a, v) \in P, g \in g^{\prime} ;\right.$ cf. 3.4(1)).

LEMMA 15. If $\Omega$ is a $P$ orbit on $\mathfrak{g}^{\prime}$, the following conditions are equivalent: (i) $\Omega / G$ is a $T_{1}$ space; (ii) there is an $O \in \Omega / G$ which is open in its closure in $g^{\prime}$.

Proof. Proceeding as in (d), proof of the previous lemma, we conclude that $\Omega$ is locally closed in $g^{\prime}$. Suppose that $O \in \Omega / G$. If the latter is a $T_{1}$ space, we have $O=\bar{O} \cap \Omega$ and thus $O$ is open in its closure. Conversely, let $O=G g$ $\subset \Omega(=P g)$ have this property. Since $\Omega$, as a subspace of $g^{\prime}$, is locally compact the map assigning ag to $a P_{g}(a \in P)$ gives rise to a $P$ equivariant homeomorphism between $P / P_{g}$ and $\Omega$. Since $L g$ is closed in $g^{\prime}, L P_{g}$ is a closed subgroup of $P$. By $\left[\right.$ S, ES] $\subseteq L$ we also have $[P, P] \subseteq L$ and hence $L P_{8}$ is invariant in $P$ such that $A=P / L P_{g}$ is abelian. Also, we have an $A$ equivariant homeomorphism between $A$ and $\Omega / L$. Let $A^{\prime}$ be the image of $G$ in $A$. If $O$ is open in its closure in $\mathfrak{g}^{\prime}, A^{\prime}$ has the same property in $A$, and thus it is closed. But then, evidently, $\Omega / G$ is a $T_{1}$-space. Q.E.D.

4. We recall (cf. 3.4(5)) that if $J \in \operatorname{Prim}(G)$ is of type one, then, in particular, $n(J)<+\infty$. The purpose of the first four points of this concluding section of Chapter II is to find an expression for this condition in terms of $\mathrm{g}^{\prime} / G$; the corresponding result is the content of Lemma 20. The final point summarizes the principal statements of this chapter (cf. Proposition 2 and Theorem 1).

4.1. In the following $\lambda$ will denote a fixed element of $\hat{L}$ which is not trivial on $N$. With the notations of $3.4(3)$, let $\lambda^{\prime \prime}$ be an element, invariant with 
respect to $\mathfrak{u}$, of $K$ such that $\lambda^{\prime \prime} \mid L=\lambda$. In the following construction we imitate the similar procedure of (d), proof of Lemma 11, to which the reader is referred for unexplained notations. Suppose that $\lambda \mid N$ gives rise to the orbit $L \pi \subset \hat{\jmath}$. Putting $K^{\prime \prime}=K_{\pi}$ we have $K=K^{\prime \prime} \cdot L$, and there exists $\rho^{\prime \prime} \in K^{\prime \prime}$ such that $\rho^{\prime \prime} \mid N$ is a multiple of $\pi$ and that $\lambda^{\prime \prime}=\operatorname{ind}_{K^{\prime \prime} \uparrow K} \rho^{\prime \prime}$. Let us write $\boldsymbol{\Omega}^{\prime \prime}=\Phi\left(K^{\prime \prime}\right) \subset \mathfrak{M}$; we can find $\mu^{\prime \prime} \in \hat{\mathfrak{\Omega}}^{\prime \prime}$ such that $\rho^{\prime \prime}=\left[\pi^{e} \otimes\left(\mu^{\prime \prime} \circ \Phi\right)\right]$. Since $\lambda=\operatorname{ind}_{L_{\mathrm{r}} \uparrow} \rho$, and $L_{\pi}=K^{\prime \prime} \cap L$ we conclude first that $\rho^{\prime \prime} \mid L_{\pi}=\rho$. Since $\rho=\left[\pi^{e} \otimes(\mu \circ \Phi)\right]^{\sim}$, we also have $\mu^{\prime \prime} \mid \mathfrak{L}=\mu$ and thus, in particular, $\mu^{\prime \prime} \mid \mathbb{Z}$ gives rise to the orbit $\mathfrak{\Omega} \subset \subset \hat{3}$. Hence there exists $\tau^{\prime \prime} \in \Omega_{\sigma}^{\prime \prime}$ such that $\tau^{\prime \prime} \mid \mathbb{Z}$ is a multiple of $\sigma$ and that $\mu^{\prime \prime}=$ ind $_{\Omega_{\sigma}^{\prime \prime} \Re^{\prime \prime}} \tau^{\prime \prime}$. By virtue of $\mathfrak{R}^{\prime \prime}=\mathfrak{Z} \cdot \mathfrak{\Omega}_{\sigma}^{\prime \prime}$, $\mathfrak{R} \cap \mathfrak{\Omega}_{\sigma}^{\prime \prime}=\mathfrak{R}_{0}$ and $\mu=\operatorname{ind}_{\mathfrak{R}_{\sigma} \uparrow \mathfrak{l}} \nu$, where $\nu \mid \mathbb{Z}$ is a multiple of $\sigma$, we infer, similarly as above, that $\tau^{\prime \prime} \mid \mathfrak{L}_{\sigma}=\nu$. Let us write $\Delta^{\prime \prime}=\varphi\left(\Omega_{\sigma}^{\prime \prime e}\right)$ and observe that, since $\mathfrak{I}_{0} \subseteq \mathfrak{\Omega}_{0}^{\prime \prime}$, we have $\mathfrak{F}^{e} \subseteq \Delta^{\prime \prime}$ and thus $\Delta^{\prime \prime}$ is invariant in $\Gamma$. Setting

$$
\tau^{\prime \prime}=\left[\sigma^{e} \otimes\left(\bar{\nu}^{\prime \prime} \circ \varphi\right)\right]^{\sim} \quad\left(\bar{\nu}^{\prime \prime} \in \hat{\Delta}^{\prime \prime}\right),
$$

by $\nu=\left[\sigma^{e} \otimes\left(\nu^{\prime} \circ \varphi\right)\right]^{\sim}$ we obtain $\bar{\nu}^{\prime \prime} \mid \mathfrak{F}^{e}=\nu^{\prime}$. In the sequel, whenever convenient, we shall write $\tau$ for $\nu^{\prime} \in \hat{\mathfrak{F}}^{e}$. Denoting again by $\Gamma_{1}$ the centralizer of $\Gamma_{0}$ in $\Gamma$ we set $\Delta=\left(\Gamma_{1}\right)_{\tau}$.

4.2. LEMMA 16. With the previous notations we have $\Delta^{\prime \prime} \subseteq \Delta$, and $\bar{\nu}^{\prime \prime}$ is invariant with respect to $\Delta$.

Proof. (a) We start by observing (cf. (c)(3), loc. cit.) that $G_{\lambda} \subseteq G_{\pi} \cdot L$, and hence also $\mathfrak{U}=\mathfrak{U}_{\pi} L$. Since $\mathfrak{U}_{\pi}=\left(\mathfrak{U}_{\pi}\right)_{\sigma} L_{\pi}$, in this manner we see that $\lambda^{\prime \prime}$ is invariant with respect to $\mathfrak{U}$ if and only if it has the same property in regard to $\left(\mathfrak{u}_{\pi}\right)_{\sigma}$.

(b) Let $\Gamma_{0}^{\natural}$ be the center of $\Gamma_{0}$ and $U$ its centralizer in $\Gamma$. We claim that $U=\Gamma_{1} \cdot \Gamma_{0}$. With the notations of (b), proof of Lemma 10, let us assume that $d \in \gamma^{1}$ is such that $(v, d) \neq 0$. As proved loc. cit., we then have $\Gamma_{1}=\Gamma_{d}$. Since evidently $\Gamma_{1} \cdot \Gamma_{0} \subseteq U$, to obtain the desired conclusion it will be enough to show that $U \subseteq \Gamma_{d} \cdot \Gamma_{0}$. To this end we first prove that $\Gamma_{0} d=d+\left(\Gamma^{q}\right)^{\perp}$. In fact, since $[\gamma, \gamma] \subseteq \mathrm{R} v$, we have

$$
\Gamma_{0} d=d+(a d \gamma)^{\prime} d=d+\left(\gamma_{d}\right)^{\perp}
$$

but $(v, d) \neq 0$ implies $\gamma_{d}=\gamma^{\natural}$. From this we conclude that if $a \in U$, then $a \Gamma_{0} d=\Gamma_{0} d$, and thus $a \in \Gamma_{d} \cdot \Gamma_{0}$, completing our proof that $U=\Gamma_{1} \cdot \Gamma_{0}$. Since $\mathfrak{F}^{e} \subseteq \Gamma_{1}$, we infer from this that $U_{\tau}=\Delta \Gamma_{0}$.

(c) We have an evident action of $\left(G_{\pi}\right)_{0}$ on $\Gamma$; we denote by $i$ the corresponding morphism $\left(G_{\pi}\right)_{\sigma} \rightarrow \operatorname{Aut}(\Gamma)$. Writing $j(\gamma)$ for the inner automorphism implemented by $\gamma \in \Gamma$ we have clearly $i\left(\left(G_{\pi}\right)_{0}\right)=j(\Gamma)$. We are going to prove now that $i\left(\left(\mathfrak{U}_{\pi}\right)_{\sigma}\right)=j\left(U_{\tau}\right)$, which statement will be used also in the next two lemmas. In fact, (1) if $a \in\left(\mathfrak{U}_{\pi}\right)_{\sigma}$, we have $a \lambda^{\prime \prime}=\lambda^{\prime \prime}$, and hence $a \rho^{\prime \prime}=\rho^{\prime \prime}$ and $a \mu^{\prime \prime}=\mu^{\prime \prime}$. Since $a \sigma=\sigma$, this implies $a \tau^{\prime \prime}=\tau^{\prime \prime}$ and $a \bar{\nu}^{\prime \prime}=\bar{\nu}^{\prime \prime}$. Therefore to 
prove that $i(a) \in j\left(U_{\tau}\right)$ it is enough to observe that, proceeding as in (d)(5), proof of Lemma 11, we can show that if $b \in \Gamma$ and $b \bar{\nu}^{\prime \prime}=\bar{\nu}^{\prime \prime}$, then $b \in U$. (2) To prove the opposite inclusion it suffices to establish that $a \in\left(G_{q}\right)_{\text {a }}$ and $i(a) \in j\left(U_{\tau}\right)$ implies $a \in\left(\mathfrak{U}_{\pi}\right)_{0}$. But (cf. loc. cit. (b), end) it follows from our assumption that $a \bar{\nu}^{\prime}=\bar{\nu}^{\prime}$ and thus $a \tau^{\prime}=\tau^{\prime}, a \rho^{\prime}=\rho^{\prime}$ and $a \lambda^{\prime}=\lambda^{\prime}$, proving that $a \in\left(\mathfrak{U}_{z}\right)_{\text {o }}$.

(d) We conclude from all this that $\bar{\nu}^{\prime \prime}$ is invariant with respect to $U_{\tau}=\Delta$. $\Gamma_{0}$ (cf. (b)). We claim that $\Delta^{\prime \prime}$ and $\Gamma_{0}$ commute. In fact, by $\left[\Gamma, \Gamma_{0}\right] \subseteq T$, for any fixed $\delta \in \Delta^{\prime \prime}$ there is a morphism $u: \Gamma_{0} \rightarrow T$ such that $\gamma^{-1} \delta \gamma=u(\gamma) \delta$. Suppose that $\bar{\nu}^{\prime \prime} \mid T$ is a multiple of $\chi \in X(T)$. If $N=\operatorname{dim}\left(\nu^{\prime}\right)$, we have, since $\bar{\nu}^{\prime \prime}$ is invariant under $\Gamma_{0}$ :

$$
(\chi(u(\gamma)))^{N} \operatorname{det}\left(\bar{\nu}^{\prime \prime}(\delta)\right)=\operatorname{det}\left(\left(\gamma \bar{\nu}^{\prime \prime}\right)(\delta)\right)=\operatorname{det}\left(\bar{\nu}^{\prime \prime}(\delta)\right),
$$

and thus $(\chi(u(\gamma)))^{N} \equiv 1$ for $\gamma \in \Gamma_{0}$. But then $\chi(u(\gamma)) \equiv 1$ and hence, by $\vec{\nu}^{\prime \prime} \in \hat{\Delta}_{-1}^{\prime \prime}, u(\gamma)=$ unity, and $\delta$ and $\gamma$ commute. Therefore, first, $\Delta^{\prime \prime} \subseteq \Gamma_{1}$, but by $\bar{\nu}^{\prime \prime} \mid \mho^{e}=\nu^{\prime}=\tau$, also $\Delta^{\prime \prime} \subseteq\left(\Gamma_{1}\right)_{\tau}=\Delta$. Q.E.D.

Given a group $A$, in the following we shall write $o(A)$ for its order.

LEMMA 17. With the previous notations, $o\left(\Delta / \Delta^{\prime \prime}\right)$ is finite if and only if $o\left(\Gamma_{1} / \Gamma_{1}^{\natural}\right)$ is.

Proof. (a) We start by observing that, since $o\left(F^{e} / T\right)<+\infty$, the set $\mathfrak{f}_{-1}^{e}$ is finite and thus $o\left(\Gamma_{1} / \Delta\right)<+\infty$.

(b) Using the maximality property of $K$ (cf. 3.4(3)) we prove next that $\Delta^{\prime \prime} \supseteq \Delta^{\natural}$. In fact, let us assume that $\Delta^{\prime \prime} \chi \Delta^{\natural}$; we can derive a contradiction from this hypothesis as follows. (1) Since $\left(\Delta^{\prime \prime}\right)_{0}=\Gamma_{0}^{\natural}=\left(\Delta^{\natural}\right)_{0}, E=\Delta^{\prime \prime} \cdot \Delta^{\natural}$ is a closed subgroup of $\Gamma$ which, by assumption, properly contains $\Delta^{\prime \prime}$. (2) Next we show that there is an $\eta \in \hat{E}$ such that $\eta \mid \Delta^{\prime \prime}=\bar{\nu}^{\prime \prime}$. In fact, let us assume that $\bar{\nu}^{\prime \prime} \mid\left(\Delta^{\prime \prime} \cap \Delta^{\natural}\right)$ is a multiple of $\varphi \in X\left(\Delta^{\prime \prime} \cap \Delta^{\natural}\right)$ and that $\chi \in X\left(\Delta^{\natural}\right)$ is an extension of $\varphi$. Then if $\delta=\delta_{1} \cdot \delta_{2}\left(\delta_{1} \in \Delta^{\prime \prime}, \delta_{2} \in \Delta^{\natural}\right)$, the value of $\bar{\nu}^{\prime \prime}\left(\delta_{1}\right) \chi\left(\delta_{2}\right)$ depends on $\delta$ only, and can be used to define the value, at $\delta \in E$, of the required $\eta \in \hat{E}$. (3) In the following we employ a construction previously considered on several occasions. Let us put

$$
\Re=q\left({ }^{-1}(E)\right) \cdot L, \quad R=h\left(\Phi^{-1}(\Re)\right) \text { and } K_{1}=R L .
$$

$K_{1}$ is a closed subgroup, properly containing $K$, of $G$. In fact, if $K_{1}=K$, we also have $R=\left(K_{1}\right)_{\pi}=K_{\pi}=K^{\prime \prime}($ cf. $4(1))$, and thus $\Re=\Omega^{\prime \prime}$ and $q\left(\bar{\varphi}^{-1}(E)\right)=$ $\Re_{\sigma}=\Re_{\sigma}^{\prime \prime}$, whence $E=\Delta^{\prime \prime}$, in contradiction to what we saw in (1). We next show the existence of $T \in \hat{K}_{1}$, such that $T \mid L=\lambda$, and which is invariant with respect to $\mathfrak{U}$. This will provide the required contradiction with the maximality of $K$. To achieve our aim let us put $\alpha=\left[\sigma^{e} \otimes(\eta \circ \varphi)\right]^{\sim} \in \hat{\mathfrak{R}}_{\sigma}$ and define successively 


$$
(\hat{\Re} \ni) \beta=\operatorname{ind}_{\Re_{0} \uparrow \Re} \alpha, \quad U=\left[\pi^{e} \otimes(\beta \circ \Phi)\right]^{\sim}, \quad T=\operatorname{ind}_{R \uparrow K_{1}} U .
$$

We claim that $T \mid L=\lambda$. In fact, we first observe that

$$
T \mid L=\operatorname{ind}_{L_{\pi} \uparrow L}\left(U \mid L_{\pi}\right) \text { and } U \mid L_{\pi}=\left[\pi^{e} \otimes((\beta \mid \mathfrak{R}) \circ \Phi)\right]^{\sim} \text {. }
$$

In this manner it will be enough to show that $\beta \mid \mathfrak{R}=\mu$ (cf. e.g. (b)(1), proof of Lemma 11). By $\eta \mid \Delta^{\prime \prime}=\bar{\nu}^{\prime \prime}$ (cf. (2)) we have $\eta \mid \mathcal{F}^{e}=\nu^{\prime}$ and thus $\alpha \mid L_{0}=\nu$. Hence the desired conclusion follows by observing that

$$
\beta \mid \mathfrak{I}=\operatorname{ind}_{\mathfrak{S}_{0} \uparrow \mathfrak{l}}\left(\alpha \mid \mathfrak{I}_{0}\right)=\operatorname{ind}_{\mathfrak{I}_{0} \uparrow \mathfrak{l}} \nu=\mu .
$$

Let us prove finally that $T$ is invariant with respect to $\mathfrak{U}$. Suppose that $a \in\left(\mathfrak{U}_{\pi}\right)_{0}$. One sees at once that upon replacing $\eta \in \hat{E}$ by $a \eta$, our construction provides $a T$ in place of $T$. Taking into account (cf. (c), proof of Lemma 16) that $i\left(\left(\mathfrak{U}_{\pi}\right)_{\sigma}\right)=j\left(U_{\tau}\right)$ and that, evidently, $\eta$ is invariant with respect to $U_{\tau}$, we conclude that $T$ is $\left(\mathfrak{U}_{\pi}\right)_{0}$ invariant which (cf. (a), loc. cit.) suffices to prove our point. Summing up, we have established that $\Delta^{\prime} \subseteq \Delta^{\prime \prime}$.

(c) We show next that $\Delta^{\prime \prime} / \Delta^{\phi}$ is finite. To this end it will be enough to prove that, given $\delta \in \Delta^{\prime \prime}$ and $\gamma \in \Delta$, there is a positive integer $M$, such that $\delta^{M}$ and $\gamma$ commute. This, however, follows from the following observations. (1) Since $[\Gamma, \Gamma] \subseteq \mathfrak{F}^{e}$ and $\mathfrak{F}^{e} / T$ is finite, if $\gamma, \delta \in \Gamma$, there is an integer $k>0$ with $\delta^{k} \cdot \gamma=\gamma \cdot \delta^{k}$. (2) Let us put $N=\operatorname{dim}\left(\nu^{\prime}\right)$. Assuming $\delta \in \Delta^{\prime \prime}, \gamma \in \Delta$ and $u=\gamma^{-1} \delta \gamma \delta^{-1} \in T$, we claim that $\delta^{N}$ and $\gamma$ commute. In fact, suppose again that $\bar{\nu}^{\prime \prime} \mid T$ is a multiple of $\chi \in X(T)$. Since, by Lemma $16, \bar{\nu}^{\prime \prime}$ is invariant with resepct to $\Delta$, proceeding as at the end of the proof loc. cit., we infer that $u=1$ implying $\delta^{N} \cdot \gamma=\gamma \cdot \delta^{N}$.

(d) We claim that $\Delta^{\natural} / \Gamma_{1}^{\natural}$ is finite. To this end it is again enough to show that if $\delta \in \Delta^{q}$ and $\gamma \in \Gamma_{1}$ then there is an integer $n>0$ such that $\delta^{n} \cdot \gamma=\gamma$. $\delta^{n}$. Using (c)(1), we can assume from the beginning that $\delta \gamma \delta^{-1}=\varepsilon \gamma(\varepsilon \in T)$. Since, by (a), $\Gamma_{1} / \Delta$ is finite, there is an integer $k>0$ such that $\gamma^{k} \in \Delta$, whence

$$
\gamma^{k}=\delta \gamma^{k} \delta^{-1}=\varepsilon^{k} \gamma^{k}
$$

and thus $\varepsilon^{k}=1$ and $\delta^{k} \cdot \gamma=\gamma \cdot \delta^{k}$. Summing up, we have

$$
\Gamma_{1} \supset \Delta \supset \Delta^{\prime \prime} \supset \Delta^{\natural} \supset \Gamma_{1}^{\natural} \text {. }
$$

By (a), $\Gamma_{1} / \Delta$ is finite; (c) and (d) imply that so is $\Delta^{\prime \prime} / \Delta_{1}^{q}$, completing the proof of our lemma. Q.E.D.

LEMMA 18. With the previous notations we have $\mathfrak{U} / K\left(G_{\lambda}\right)_{0}=\Delta / \Delta^{\prime \prime}$.

Proof. We start by observing that $\Delta \cap \Gamma_{0}=\Delta^{\prime \prime} \cap \Gamma_{0}$. In fact, to this end it is enough to note that

$$
\Gamma_{0}^{\natural}=\Gamma_{1} \cap \Gamma_{0} \supseteq \Delta \cap \Gamma_{0} \supseteq \Delta^{\prime \prime} \cap \Gamma_{0} \supseteq \Gamma_{0}^{\natural}
$$


In this manner we can conclude that

$$
\Delta / \Delta^{\prime \prime}=\Delta \Gamma_{0} / \Delta^{\prime \prime} \Gamma_{0}=U_{\tau} / \Delta^{\prime \prime} \Gamma_{0}=q\left(\varphi^{-1}\left(U_{\tau}\right)\right) \cdot \mathfrak{L} / \mathfrak{R}^{\prime \prime}\left(\mathfrak{M}_{\sigma}\right)_{0} .
$$

We recall (cf. (d)(4), proof of Lemma 11) that if $H=\left(G_{\lambda} \cap G_{\pi}\right)_{0}$, we have $\Phi\left(H^{e}\right)=\left(\mathfrak{R}_{\mathrm{o}}\right)_{0}$. Therefore, by what we saw in (c), proof of Lemma 16, we infer that

$$
\Delta / \Delta^{\prime \prime}=\left(\mathfrak{U}_{\pi}\right)_{\sigma} L_{\pi} / K^{\prime \prime} H=\mathfrak{U} / K\left(G_{\lambda}\right)_{0}
$$

completing the proof of our main assertion. Q.E.D.

4.3. The purpose of the following will be the definition of the notion of the reduced stabilizer of an element $g \in W(G)$. In the special case, when $G$ is solvable, this was discussed in [18, Definition 4.1, p. 492]. Let us put $\mathcal{G}_{g}^{\prime}=p^{-1}\left(\left(G_{g}\right)_{0}\right)$. Then (cf. 2.1) there is a $\chi_{g}^{\prime} \in X\left(\mathcal{G}_{g}^{\prime}\right)$ such that $\chi_{g}^{\prime} \mid\left(\mathcal{G}_{g}\right)_{0}=\chi_{g}$, $\chi_{8}(\varepsilon)=-1$. Writing for $a, b \in G:[a, b]=a b a^{-1} b^{-1}$, we set

$$
\overline{\mathcal{G}}_{\mathrm{g}}=\left\{a ; a \in \mathcal{G}_{\boldsymbol{g}},[a, b] \in \operatorname{ker}\left(\chi_{\boldsymbol{g}}^{\prime}\right) \text { for all } b \in \mathcal{G}_{\mathbf{g}}\right\} \text {. }
$$

DEFINITION. With the previous notations, we define the reduced stabilizer $\bar{G}_{g}$ of an admissible element $g \in g^{\prime}$ by $p\left(\overline{\mathcal{G}}_{g}\right)$.

We observe that $\bar{G}_{g}$ is an open subgroup in $G_{g}$. In fact, to see this it is enough to remark that since $\chi_{g}^{\prime}$ is invariant with respect to $\mathcal{G}_{g}$, we have $\mathcal{G}_{g} \supseteq \mathcal{G}_{g}^{\prime}$. It is also of some interest to bear in mind that $\bar{G}_{g}$ depends on $g \mid b$ only, the verification of which we leave to the reader.

4.4. We continue to denote (cf. 4.1) by $\lambda$ a fixed element of $\hat{L}$ which is not trivial on $N$.

Lemma 19. Let $g \in W(G)$ be such that $g \mid \mathfrak{b} \in \eta(\lambda)$ (cf. Lemma 6). With the notations of 4.2 and 4.3 we have $\Gamma_{1} / \Gamma_{1}^{\natural}=G_{g} / \bar{G}_{g}$.

Proof. (a) Using the notations of Lemma 10 let us show first that there is a $d \in \gamma^{\prime}$ such that $\delta \Lambda(d)=g \mid a$ and $(v, d) \neq 0$. We write again $h=g \mid d$ and set, as in the proof of Lemma $3, M=\left(L_{h}\right)_{0} N_{f}$ and $\Re={ }^{-1}(M) \subset \mathcal{P}$. We recall also (cf. loc. cit.) that $\Re=\Psi^{-1}\left(3_{\kappa}\right)$. Again forming $\chi=\chi_{*} \circ(\Psi \mid \Re)$ one sees at once that $d \chi=i(g \mid \mathfrak{m})\left(\mathfrak{m}=\delta_{h}+\mathfrak{n}_{f}\right)$ and that $\operatorname{ker}(\Lambda)=\operatorname{ker}(\chi)$. In this manner $\operatorname{ker}(d \Lambda)=\operatorname{ker}(g \mid \mathfrak{m})$ and thus there is a $d \in \gamma^{\prime}$ with $\delta \Lambda(d)=g \mid a$. Since $\Lambda^{-1}(T)=\mathscr{R}$, if $(v, d)=0$, we have $g \mid \mathfrak{m}=0$. Since $f=g \mid \mathfrak{n}$, this implies that $f \mid \mathfrak{n}_{f}=0$ and thus $f=0$; hence $\operatorname{ker}(\lambda) \supset N$ (cf. (a), proof of Lemma 6), which, however, contradicts our assumption on $\lambda$. By what we saw in Lemma 10 , we have $\Gamma_{1}=\Gamma_{d}$ and $\Lambda^{-1}\left(\Gamma_{d}\right)=\mathcal{G}_{g} \Re$. Let us set

$$
\mathscr{B}=\left\{a ; a \in \mathcal{G}_{\boldsymbol{g}} \mathfrak{R},[a, b] \in \operatorname{ker}(\chi) \text { for all } b \in \mathcal{G}_{\boldsymbol{g}} \mathfrak{R}\right\} \text {. }
$$


Summing up, we have

(b) Writing

$$
\mathscr{B}=\bar{\Lambda}^{-1}\left(\Gamma_{1}^{\mathfrak{q}}\right) \text { and } \Gamma_{1} / \Gamma_{1}^{\mathfrak{q}}=\mathcal{G}_{8} \Re / \mathscr{B}
$$

$$
\mathscr{F}=\left\{a ; a \in \mathcal{S}_{g},[a, b] \in \operatorname{ker}(\chi) \text { for all } b \in \mathcal{G}_{g}\right\}
$$

we clearly have $\mathscr{B}=\mathscr{F} \cdot \mathscr{T}$. We next show that $\mathscr{F}=\overline{\mathcal{G}}_{g}$. To this end we first recall (cf. (c), loc. cit.) that $\mathfrak{M} \cap G_{g}$ is connected. Therefore, if $a \in \mathcal{F}$ and $b \in \mathcal{G}_{g}$, we have

$$
[a, b] \in \operatorname{ker}(\chi) \cap \mathcal{G}_{g} \subset \mathscr{T} \cap \mathcal{G}_{g}=p_{p}^{-1}\left(M \cap G_{g}\right) \subset \stackrel{-1}{p}^{-1}\left(\left(G_{g}\right)_{0}\right)=\mathcal{G}_{g}^{\prime} \text {. }
$$

Since evidently $\chi\left|\left(\mathfrak{N} \cap \mathcal{G}_{g}\right)=\chi_{g}^{\prime}\right|\left(\mathfrak{R} \cap \mathcal{G}_{g}\right)$, this implies $a \in \overline{\mathcal{G}}_{g}$ and thus $\mathscr{F} \subseteq \overline{\mathcal{G}}_{\boldsymbol{g}}$. To establish the opposite inclusion it is enough to prove that $a$, $b \in \mathcal{S}_{g}$ and $[a, b] \in \operatorname{ker}\left(\chi_{g}^{\prime}\right)$ imply $[a, b] \in \mathfrak{R}$. This, however, follows from

$$
[p(a), p(b)] \in\left(G_{g}\right)_{0} \cap L=\left(L_{g}\right)_{0} \subseteq\left(L_{h}\right)_{0} N_{f}=M \text {. }
$$

(c) Bearing in mind that $G_{g} \cap M=\bar{G}_{g} \cap M$, we infer from what we have just seen that

$$
\Gamma_{1} / \Gamma_{1}^{\natural}=\mathcal{G}_{g} \Re / \overline{\mathcal{G}}_{g} \mathfrak{N}=G_{g} M / \bar{G}_{g} M=G_{g} / \bar{G}_{g}
$$

completing the proof of Lemma 19. Q.E.D.

We observe that the (not necessarily finite) order $o\left(G_{g} / \bar{G}_{g}\right)$ of $G_{g} / \bar{G}_{g}$ depends on $O=G g$ only; hence we may write it as $i(O)$. We recall (cf. 3.4(5)) that, given $J \in \operatorname{Prim}(G)$, by definition $n(J)=o\left(\mathfrak{H} / K\left(G_{\lambda}\right)_{0}\right) \quad \lambda \in$ $E(J))$. For $\Omega(J)$, cf. Lemma 14.

LEMMA 20. For any $J \in \operatorname{Prim}(G)$, the following conditions are equivalent: (i) $n(J)<+\infty$; (ii) for any $O \in \Omega(J) / G, i(O)<+\infty$.

Proof. Let us select a $g \in g^{\prime}$, such that $g \in \Omega(J)$ or $g \mid \mathfrak{d} \in \eta(E(J))$. If $g \mid \mathfrak{n}=0$, we have $\operatorname{ker}(\lambda) \supseteq N$ for all $\lambda \in E(J)$ and conversely; but then $\boldsymbol{n}(J)=i(O)=1(O=G g)$. Let us now assume that $g \mid \mathfrak{n} \neq 0$. We then have, by Lemma $18, o\left(\Delta / \Delta^{\prime \prime}\right)=n(J)$ and, by Lemma $19, o\left(\Gamma_{1} / \Gamma_{1}^{q}\right)=i(O)$. Finally, Lemma 17 implies that if one of the expressions on the left-hand side is finite, then so is the other. Q.E.D.

4.5. We are going to summarize the principal results of Chapter II in the following fashion.

Proposition 2. Suppose that $G$ is a connected and simply connected Lie group with the Lie algebra $\mathrm{g}$. Let us assume that the radical of $\mathrm{g}$ is cocompact. Then the following conditions bearing on $J \in \operatorname{Prim}(G)$ are equivalent: (i) $J$ is of type I; (ii) if $O \in \Omega(J) / G, O$ is open in its closure and $i(O)<+\infty$. 
Proof. Let us first assume that $J$ is of type I and that $O \in \Omega(J) / G$. We claim that $O$ is open in its closure. In fact, by what we said in 3.4(5), our assumption implies that $F(J) / G$ is a $T_{1}$ space whence, by Lemma 14 , so is $\Omega(J) / G$, and thus the desired conclusion follows from Lemma 15. Also, our hypothesis yields (cf. loc. cit.) that $n(J)<+\infty$ and therefore, by Lemma 20, $i(O)<+\infty$. The opposite implication can be established in a similar fashion. Q.E.D.

THEOREM 1. Suppose that $G$ is a connected and simply connected Lie group with the Lie algebra $g$, the radical of which is cocompact. $G$ is of type one if and only if any $G$ orbit $O$ in $W(G) \subset g^{\prime}(c f .2 .1)$ is open in its closure and $i(0)<+\infty$.

Proof. Let us first assume that $G$ is of type $I$. By definition this means that any factor representation of $G$ generates a factor of type I. But then (cf. 3.4(5) and [20, Lemma 3.1, p. 110]) any primitive ideal is of type I, and conversely. Suppose that $O$ is a $G$ orbit in $W(G)$. We claim that there is a $J \in \operatorname{Prim}(G)$ such that $O \subset \Omega(J)$. In fact, putting $D=O \mid \delta$, by Lemma $7, \mathfrak{D} \in W(L) / G$. If $h$ is some element of $D$, by Lemma 6 we can find $\lambda \in \hat{L}$ with $h \in \eta(\lambda)$. Thus, to arrive at the desired conclusion it is enough to note that $\hat{L}=$ $\cup_{J \in \operatorname{Prim}(G)} E(J)$. In this manner, assuming that $J$ is of type I, Proposition 2 implies that $O$ is open in its closure and $i(O)<+\infty$. Conversely, suppose that this pair of conditions is fulfilled for any $G$ orbit in $W(G)$. Let $J$ be some element in $\operatorname{Prim}(G)$. Since $\Omega(J) \subset W(G)$, by Proposition $2, J$ is of type $I$. Hence, by our opening observation $G$ is also of type I. Q.E.D.

Corollary (Auslander AND KostaNt). Suppose that $G$, as in Theorem 1, is also solvable. Then $G$ is of type $\mathrm{I}$ if and only if any $G$ orbit $O$ in $g^{\prime}$ is open in its closure and $i(O)$ is identically zero.

In fact, by virtue of what we said in 2.1 , here $W(G)=g^{\prime}$. Hence it suffices to observe that, trivially, if $i(O)$ is always finite, then it is identically zero.

\section{LIE GROUPS WITH COMPLETELY CONTINUOUS REPRESENTATIONS}

As already stated in the Introduction, the principal objective of this closing chapter is to obtain, as an application of our previous results, a characterization of all connected and simply connected CCR Lie groups; the corresponding result is contained in Theorem 2. To accomplish this, we are first going to establish the equivalence of a series of properties bearing on a connected Lie group (cf. Proposition 3) which is of a certain independent interest. The final conclusion will be derived by combining part of this with Theorem 1. 
1.1. Suppose that $M$ is a semifinite factor and $\Phi$ is a faithful normal trace on $\mathrm{M}^{+}$( = set of all positive elements of $M$ ). We shall denote in the same manner the corresponding linear form, defined on the (dense) 2-sided ideal, composed of all linear combinations of elements of $\mathrm{M}^{+}$with a finite trace. We shall say that $A \in M$ is a generalized completely continuous operator $(A=C C)$ if so is $|A| ; A \in M^{+}$with the spectral representation $\int_{0}^{+\infty} \lambda d E_{\lambda}$ will be said to have this property if, for any $\lambda>0, \Phi\left(I-E_{\lambda}\right)<+\infty$. We denote the set of all such operators by $C(M)$. Let $G$ be a separable locally compact group and $\mathcal{G}$ its group $C^{*}$ algebra. We recall (cf. [20, p. 81, bottom]) that a factor representation $U$ of $G$ is called normal if, putting $M=R(T)$ (A.8), $U(\mathcal{G}) \cap C(M) \neq\{0\}$. We write $\operatorname{Facn}(G)$ for the set of all such representations. For the following it is of some interest to bear in mind that, if $G$ is a connected Lie group, given $J \in \operatorname{Prim}(G)$, there is an element $U$, uniquely determined up to quasi-equivalence, of $\operatorname{Facn}(G)$, such that $\operatorname{ker}(U)$ $=J$ (cf. [20, Theorem 1, p. 119]), but we shall not use this in our proofs. We shall say that a factor representation $T$ of $G$ is a generalized completely continuous representation if $T(\mathcal{G}) \subset C(R(T))$. Let us observe that, if $T$ is irreducible, this is the case if and only if the range of $T$ is contained in the set of all completely continuous operators (in the classical sense) of the representation space, in which case we call it a CCR representation. The previous definitions can be adopted, in an obvious manner, to an arbitrary separable $C^{*}$ algebra $A$. Any representation to be considered in the sequel will be assumed to be nondegenerate.

1.2. LEMMA 21. With the previous notations suppose that $T$ is a normal representation such that $\operatorname{ker}(T)$ is maximal. Then $T$ is a generalized completely continuous representation.

Proof. Let us put $M=R(T)$ (A.8); our assumption implies that $M$ is a semifinite factor.

(a) Let us note first that $C(M)$ is a uniformly closed 2-sided ideal of $M$. To see this it is enough to show that if $A \in M$ with $0<A<I$ is a uniform limit of $\left\{A_{n}\right\} \subset C(M)$, such that $0<A_{n}<I$, then $A \in C(M)$. We can assume also that $\Phi\left(A_{n}\right)<+\infty$. In fact, if $A_{n}=\int_{0}^{1} \lambda d E_{\lambda}^{(n)}$, it suffices to replace $A_{n}$ through $A_{n}\left(I-E_{1 / n}^{(n)}\right)(n=1,2, \ldots)$. Suppose that $A=\int_{0}^{1} \lambda d E_{\lambda}$. We have to show that, if $\alpha$ is fixed between zero and one and $P=I-E_{\alpha}$, then $\Phi(P)<+\infty$. Let us put $B_{n} \equiv P A_{n} P$. Since $\left\|P-B_{n}\right\|<(1-\alpha)+\| P A-$ $B_{n} \|$, the restriction of $B_{n}$ to the subspace of $P$ admits a bounded relative inverse for a sufficiently large $n$. But, since $\Phi\left(B_{n}\right)<+\infty$, this implies $\Phi(P)<+\infty$.

(b) Let us put $\mathfrak{I}=T(A)$ and $\mathfrak{\Im}_{1}=I \cap C(M)$. To complete the proof of our lemma we have to show that $\mathfrak{\Im}_{1}=\mathfrak{\Im}$. By what we saw in (a), $\mathfrak{\Im}_{1}$ is a norm 
closed 2 -sided ideal in $\mathfrak{\Im}$. Since $T$ is normal, $\mathfrak{\Im}_{1}$ is nonzero, hence its complete inverse image in $A$ properly contains $\operatorname{ker}(T)$. But the latter being maximal by assumption, we necessarily have $\mathfrak{\Im}=\mathfrak{\Im}_{1}$. Q.E.D.

2.1. In the sequel the following notations will be useful. Given a locally compact group $K$, we shall write $K=(P)$ if $K / K_{0}$ is finite (cf. A.6). Let $g$ be a Lie algebra of endomorphisms of a finite dimensional real vector space $V$. If $G=\exp (g)$ (cf. A.4), we shall write $G^{\prime}$ for the analytic subgroup, corresponding to $g$, of $G L(V)$. We recall that $g$ is called algebraic, if $G^{\prime}$ is the connected component of the identity of an algebraic subgroup of $G L(V)$. In this case, by virtue of a theorem due to J. Dixmier (cf. [7, Proposition, p. 425, bottom]), $G$ is of type I.

LEMMA 22. Suppose that $\mathrm{g}$ and $\mathfrak{h}$ are algebraic Lie algebras of endomorphisms of some finite dimensional real vector space such that $\mathfrak{h}$ is an ideal in $g$. Let $\lambda$ be some element in $\hat{H}(H=\exp (\mathfrak{h}))$. Then with notations as above, $G_{\lambda}^{\prime}=(P)$.

Proof. The subsequent reasonings will have many points in common with those of [19], to which paper we shall often refer for further details. Let us put $\boldsymbol{G}=\exp (\mathrm{g})$. We shall proceed by induction and assume that our statement is valid for any similar pair $\left(H_{1}, G_{1}\right)$ if $\operatorname{dim}\left(G_{1}\right)<\operatorname{dim}(G)$. Let us denote by $m$ the collection of all nilpotent endomorphisms in the radical of $\mathfrak{h} ; \mathfrak{m}$ is the greatest ideal of nilpotency of the identity representation of $\mathfrak{h}$ (cf. loc. cit., $p$. 385 , bottom). We can assume that $\mathfrak{m}$ is nontrivial. In fact, otherwise $\mathfrak{h}$ is reductive (cf. loc. cit., p. 389 ) and $\left(G^{\prime}\right)_{\lambda}$ is an algebraic subgroup of $G^{\prime}$. We shall distinguish two cases, according to whether: (A) There is an abelian ideal $a$, contained in $m$, of $g$ such that either $\operatorname{dim}(a)>1$ or, if $\operatorname{dim}(a)=1$, we have $a \not \subset g^{\natural}$ (A.7). (B) Condition (A) cannot be fulfilled. Ad(A) Let us put $A=\exp (\mathrm{a}) \subset H$ (A.4). $A$ is closed, simply connected and invariant in $H$, hence $\hat{A} / H$ is countably separated (cf. loc. cit., p. 386). Therefore $\lambda$, on $A$, restricts to a transitive quasi-orbit, $H \omega$ (say), in $\hat{A}$. We next divide our discussion into two parts: (A1) $\omega$ is not $G$ invariant or (A2) $\omega$ is $G$ invariant. Ad (A1) Let us put $S=H_{\omega}$; its Lie algebra is algebraic and $S^{\prime}=(P)$ (cf. loc. cit., p. 387, top). By what we saw above there is a $\rho \in \hat{S}$ such that $\rho \mid A$ is a multiple of $\omega$ and $\lambda=\operatorname{ind}_{S \uparrow H} \rho$. One easily verifies that $G_{\lambda}=\left(G_{\omega}\right)_{\lambda} H$.We also have $G^{\prime}=(P)$ (cf. loc. cit.). Therefore, putting $F=\left(G_{\omega}\right)_{0}$ it is enough to show that $F_{\lambda}^{\prime}=(P)$. Observe next that $F_{\lambda}=F_{\delta}$. The restriction of $\rho$ to $S_{0}$ gives rise to a finite $S$ orbit, $S_{\sigma}\left(\subset \hat{S}_{0}\right)$ say, transformed into itself by the action of $F_{\rho}$ on $\hat{S}_{0}$. Hence $F_{\rho} /\left(F_{\rho} \cap F_{\sigma}\right)$ is finite and $\left(F_{\rho}\right)_{0}=\left(F_{\rho} \cap F_{\sigma}\right)_{0}$. We claim that $F_{\sigma} /\left(F_{\rho} \cap F_{\sigma}\right)$ is also finite. In fact, let $\varphi$ be the canonical homomorphism $G \rightarrow G^{\prime}$ and let us put $\Gamma=\operatorname{ker}(\varphi) \cap S\left(\subset G^{\natural}\right)$. We write $\mathcal{H}=\Gamma \cdot S_{0}$ and observe that by $S^{\prime}=(P), S / \mathcal{H}$ and $S / S_{0}$ are finite. Let $\tau \in \hat{S}_{\circ}$ be such that $\tau \mid S_{0}$ is a multiple of $\sigma$ and $\rho=\operatorname{ind}_{S_{\diamond} \uparrow S} \tau$. Since $\tau \mid \mathcal{H C}$ is $F_{\circ}$ 
invariant, $F_{\sigma} \tau \subset \hat{S}_{\sigma}$ is finite and thus so is $F_{o} \rho$, and hence $F_{\sigma} /\left(F_{\rho} \cap F_{\sigma}\right)$ is finite. We infer from this that $\left(F_{\sigma}\right)_{0}=\left(F_{\rho} \cap F_{\sigma}\right)_{0}=\left(G_{\rho}\right)_{0}$. In this manner to prove that $F_{\rho}^{\prime}=(P)$ it suffices to show that $F_{\sigma}^{\prime}=(P)$. This we derive from the assumption of our inductive procedure in the following manner. Let $\tilde{S}$ and $\tilde{F}$, respectively, be the universal coverings of $S_{0}$ and $F$. They are locally isomorphic to algebraic subgroups of $G L(V)$. By virtue of the hypothesis (A1) we have $\operatorname{dim}(\tilde{F})<\operatorname{dim}(G)$. Let $\zeta$ be $\sigma$ lifted to $S$. Then we can conclude that $\tilde{F}_{\zeta}^{\prime}=(P)$ and hence also $F_{o}^{\prime}=(P)$. $\operatorname{Ad}(\mathrm{A} 2)$ Let us denote by $J$ the connected component of the identity in the kernel of $\omega$. By assumption, $J$ is an invariant subgroup of $G$ and we claim that its dimension is positive. This is clear if $\operatorname{dim}(\mathfrak{a})>1$. If, however, $\operatorname{dim}(\mathfrak{a})=1$, by hypothesis, $\mathfrak{a}$ is not contained in the center of $g$. Therefore $\omega$ can be invariant with respect to $G$ only if it is the identity character, implying $J=A$. Let $\mathrm{i}$ be the subalgebra of a corresponding to $J$. By what we have just seen, $i$ is a $g$ ideal contained in $m$ and therefore (cf. loc. cit., p. 389$) \mathrm{g} / \mathrm{i}$ is isomorphic to an algebraic Lie algebra $g_{1}$. We denote by $\mathfrak{h}_{1}$ the image of $\mathfrak{h} / \mathfrak{i}$ and form $\exp \left(g_{1}\right)=G_{1} \supseteq H_{1}=\exp \left(\mathfrak{h}_{1}\right)$. We have $\operatorname{dim}\left(G_{1}\right)<\operatorname{dim}(G)$ and $\lambda \in \hat{H}$ is obtained by lifting some $\lambda_{1} \in \hat{H}_{1}$ to $H$. Hence the desired conclusion results by applying the assumption of our induction to $\left(H_{1}, G_{1}\right)$ and $\lambda_{1}$. (Ad)(B) This can be settled by a simple adaptation of the reasoning of $[7$, p. 389 , loc. cit.], and hence we confine ourselves to recalling the principal steps of the argument. (1) We have $[\mathfrak{m}$, $\mathfrak{m}] \subseteq \mathfrak{m}^{q}$ and $\operatorname{dim}\left(\mathfrak{m}^{\mathfrak{g}}\right)=1$; we put $M=\exp (\mathfrak{m})$. (2) There is an ideal $g_{1}$ of $\mathfrak{g}$ such that $\mathfrak{g}=\mathfrak{h}+\mathfrak{g}_{1}$ and $\left[\mathfrak{g}_{1}, \mathfrak{h}\right] \subseteq \mathfrak{m}$. Hence, putting $G_{1}=\exp \left(g_{1}\right)$, we have $G_{\lambda}=\left(G_{1}\right)_{\lambda} \cdot H$. (3) One can assume that $\lambda \mid M^{\natural}$ is not constant; in fact, otherwise, reasoning as in (A2), one can effect an immediate reduction. (4) By (1), $\lambda \mid M$ is a multiple of some $\omega \in \hat{M}$; we assume that it belongs to the Kirillov orbit $M f \subset \mathfrak{m}^{\prime}$. (5) We have $G_{1}=\left(G_{1}\right)_{\omega}=\left(G_{1}\right)_{f} \cdot M$; hence, putting $T=\left(G_{1}\right)_{f}$, by (2), it will be enough to show that $T_{\lambda}$ is connected. (6) Let us form $K=H / M ; K$ is reductive. Let $\omega^{\prime}$ be a projective extension of $\omega$ to $H$. Then there is a continuous morphism $\chi: T \rightarrow X(K)$ such that $b \omega^{\prime}=$ $i(\chi(b)) \omega^{\prime}$, where, for $\varphi \in X(K), i(\varphi)$ stands for the corresponding element of $X(H)$. (7) We denote by $\hat{S}_{\omega}$ the set of all elements of $\hat{H}$, the restriction of which to $M$ is a multiple of $\omega$. We have $\lambda \in \hat{S}_{\omega}$. Let $\Lambda$ be the set of all class minus one elements of the dual of the extension, by the circle group, of $K$ ( $K_{a}$, say), corresponding to the obstruction class of $\omega^{\prime}$. Then there is a Borel isomorphism $\varphi: \Lambda \rightarrow \hat{S}_{\omega}$, such that $b \varphi(\tau)=\varphi\left(\chi^{\prime}(b) \tau\right)$, where $\chi^{\prime}(b)$ is equal to $\chi(b)$ lifted to $K_{a}(b \in T, \tau \in \Lambda)$. (8) We can write $K_{a}$ as $D \times A_{1} \times A_{2}$, where $D$ is semisimple, $A_{1}$ is an extension of a vector group by the circle group, such that $\operatorname{dim}\left(A_{1}^{4}\right)=1$, and $A_{2}$ is a vector group. Accordingly, $\chi^{\prime}(b)(b \in T)$ can be written as $1 \times \chi_{1}(b) \times \chi_{2}(b)$. The map $b \rightarrow \chi_{2}(b)$ is a morphism of $T$ into $X\left(A_{1}\right)$ and we have $T_{\lambda} \ni b$ if and only if $\chi_{2}(b)$ is the unit character proving that $T_{\lambda}$ is connected. Q.E.D. 
We recall that any Lie algebra $g$ admits a unique maximal semisimple ideal, $\subseteq$ say, which is a direct factor. We shall say that $g$ has no semisimple factors $(g=$ n.s.f.) if $\mathfrak{S}=0$. Let $r$ be the radical of $g$. We note that the preceding condition is fulfilled if and only if the centralizer of $r$ in $g$ is equal to the center of $r$. We recall that if $g$ is solvable its roots, by definition, are the complex-valued linear forms corresponding to the irreducible quotient modules of its adjoint representation. $g$ is said to be of type $R(g=(R))$ if its roots are purely imaginary.

The following reasoning is partly inspired by [12] (cf. proof of Theorem 3.1, p. 744) and [2, pp. 171-172], and has points in common with [14] (cf. proofs of Propositions 2.1-2.3 loc. cit.). Parts of the subsequent arguments will be used also in the proof of Lemma 24.

Lemma 23. Suppose that $\mathrm{g}$ has no semisimple factors. Putting $G=\exp (\mathrm{g})$ assume also that any irreducible normal representation of $G$ is $C C R$ (cf. 1.1). Then the radical $\mathrm{r}$ of $\mathfrak{g}$ is of type $R$ and $\mathfrak{g} / \mathrm{r}$ is compact.

Proof. If our claim fails to be correct, then either (A) $r$ is not of type $R$, or (B) $\mathfrak{r}$ is of type $R$ but $\mathrm{g} / \mathrm{r}$ is not compact. In each of these cases we shall exhibit a type I factor group of $G$ which is not CCR. Then a contradiction will be obtained by recalling that for a type I group any irreducible representation is normal (cf. [6, 9.1, Théorème, (ii) $\Rightarrow$ (iv), p. 168]).

$\operatorname{Ad}(A)(a)$ First we show the existence of a factor algebra $f$ of $g$ such that its radical is not of type $R$, but its nilradical is a minimal ideal. (1) We recall that if $\mathfrak{n}$ is nilpotent and the direct sum of a subspace $\mathfrak{a} \subset \mathfrak{n}$ and of $[\mathfrak{n}, \mathfrak{n}]$, then $a$ generates $\mathfrak{n}$. Now let $\mathfrak{n}$ be the nilradical of $\mathfrak{g}$. We conclude from our observation that the radical of $\mathrm{g} /[\mathrm{n}, \mathrm{n}]$ is not of type $R$, and that its nilradical is equal to $\mathfrak{n} /[\mathfrak{n}, \mathfrak{n}]$. Therefore we can assume from the beginning that $\mathfrak{n}$ is an abelian $\mathfrak{r}$ module. (2) Let $\mathcal{F}$ be the set of all roots of $r$. Then $\mathfrak{n}$ is a direct sum $\Sigma_{\gamma \in \mathscr{F}} \mathfrak{n}_{\gamma}$, where $\mathfrak{n}_{\gamma}$ is a $\gamma$ pure $r$ module (cf. [3, p. 41 and (12), p. 127]). We also recall that $n_{\gamma}$ is a $g$ ideal (cf. loc. cit., Proposition 1, p. 41). Suppose that $\alpha$ is not purely imaginary. Then putting $\mathfrak{m}=\Sigma_{\gamma \neq \alpha} \mathfrak{n}_{\gamma}$ and dividing through $\mathfrak{m}$ we can assume that $r$ has only one nonzero root and is not of type $R$. (3) This being so, $\mathfrak{n}$ is a direct sum $\mathfrak{n}_{0}+\mathfrak{n}_{\alpha}$, where $\mathfrak{n}_{0}$ is the largest subspace of $\mathfrak{n}$, on which $\operatorname{ad}(\mathfrak{r})$ is nilpotent. $\mathfrak{n}_{0}$ is a $g$ ideal, and by forming the corresponding quotient we can assume that $\mathfrak{r} \neq(R)$ and $\mathfrak{n}$ is $\alpha$ pure. (4) Finally let $m$ be a maximal proper $g$ ideal in $\mathfrak{n}$. Dividing through it we obtain a factor algebra $f$ of our starting algebra $g$ having all the properties listed at the start of (a). For later use observe that $f$ can be obtained by taking the quotient of $g$ according to an ideal contained in the radical.

(b) Putting $F=\exp (\mathfrak{f})$ (A.4) we claim that $F$ is of type I. In the following, to simplify our notations we shall write $f=(a)$ if $f$ is isomorphic to an 
algebraic Lie algebra of endomorphisms. We recall that this is the case if and only if the radical of $f$ admits the said property. Let $\mathfrak{r}$ be the radical, $\mathfrak{n}$ the nilradical of $f$ and $\alpha=\alpha_{1}+i \alpha_{2}$ the only nonzero root of $r$. We distinguish three possibilities: (1) $\alpha_{1}$ and $\alpha_{2}$ are linearly independent, (2) $\alpha_{2} \equiv 0$, (3) $\alpha_{1} \equiv c \alpha_{2}, c \neq 0$. Observe that, since $n$ is a minimal $g$ ideal, the restriction of $\operatorname{ad}(r)$ to $n$ is composed of semisimple endomorphisms. Hence in cases (1) and (2) we clearly have $r=(a)$, and thus $f=(a)$ implying that $F$ is of type I (cf. [7, Proposition, p. 425]). As regards case (3), let $\mathfrak{h}$ be a Levi section in $f$. We write $b=\mathfrak{h}+\mathfrak{n}=[\mathfrak{f}, \mathfrak{f}]+\mathfrak{n}$ and observe that since $\delta=(a), L=\exp (\delta)$ is a closed, type $I$, invariant subgroup in $F$. By $\operatorname{dim}(f / \delta)=1$, to prove that $F$ is of type I it suffices to show that $\hat{L} / F$ is countably separated. We can assume that $r$ is a direct sum $a+\mathfrak{n}$, where $\operatorname{dim}(\mathfrak{a})=1$ and $[\mathfrak{h}, \mathfrak{a}]=0$. Since $\mathfrak{n}$ is $\alpha$ pure and $\alpha \neq 0$, the center of $f$ is trivial and thus we can identify $f$ with $\operatorname{ad}(f)$. Let $\mathfrak{A}$ be the smallest algebraic Lie algebra of endomorphisms of $f$ containing a. We have $\operatorname{dim}(\mathfrak{A})=2,[\mathfrak{A}, \mathfrak{n}] \subseteq \mathfrak{n}$ and $[\mathfrak{A}, \mathfrak{h}]=0$. Therefore $\mathfrak{M}=\mathfrak{h}+\mathfrak{A}+\mathfrak{n}$ is a Lie algebra containing $d$ as an ideal and $\mathfrak{m}=(a)$. Let us put $\mathfrak{m}=\exp (\mathfrak{m})$ $\supset \mathbb{Q}=\exp (\mathfrak{U})$. Then $\hat{L} / M=\hat{L} / \mathbb{Q}$ is countably separated (cf. [19, Theorem, p. 379]). Hence, for any $\tau \in \hat{L}, Q \tau$ is locally closed in $\hat{L}$ (cf. [9, Theorem 1 , (3) $\Rightarrow(1)$, p. 124]). Let us write $A=\exp (a) \subset F$. To show that $\hat{L} / F$ is countably separated, it is enough to prove that $F \tau=A \tau$ is locally closed in $\hat{L}$ (cf. loc. cit. (1) $\Rightarrow(3)$ ). As in our previous lemma, let us again set $M^{\prime}=$ $\exp (\mathfrak{m}) \subset G L(\mathfrak{f})$, etc. We have a natural homeomorphism between $\mathbb{Q}_{\tau}=M \tau$ $\subset \hat{L}$ and, respectively, $M^{\prime} / M_{\tau}^{\prime}$ and $\mathcal{Q}^{\prime} / \mathcal{Q}_{\tau}^{\prime}$ (cf. loc. cit. (3) $\Rightarrow(6)$ ). Hence, by Lemma 22 applied to $m$ and $b$ in place of $\mathfrak{g}$ and $\mathfrak{h}$ loc. cit., $\mathbb{Q}^{\prime} \tau$ has finitely many connected components. Since $Q^{\prime}$ is isomorphic to the product of the circle group with the line, $A^{\prime} \cdot Q_{\tau}^{\prime}$ is closed in $\mathcal{Q}^{\prime}$. Therefore $F_{\tau}=A \tau$ is closed in $Q_{\tau}$ and thus locally closed in $\hat{L}$.

(c) Next we show that $F$ has an irreducible representation which is not CCR; as indicated at the start this implies a contradiction with the assumptions of our lemma. For the following reasoning cf. [12, p. 746]. Let us put $H=\exp (\mathfrak{h}) \subset F$ and $K=H A ; F$ is the semidirect product of the closed subgroups $K$ and $N$. We denote by $\chi_{0}$ the unit character of $X(N)$. Since $\mathbf{r} \neq(R)$, there is a $\chi \in X(N)$ and a sequence $\left\{a_{n}\right\} \subset A$ such that $\left\{a_{n} \chi\right\}$ converges to $\chi_{0}$ in $X(N)$, but $a_{n} \chi \neq \chi_{0}(n=1,2, \ldots$,$) . We have F_{\chi}=K_{\chi}$. $N$, and there is $\varphi \in X\left(F_{\chi}\right)$ such that $\varphi \mid K_{\chi} \equiv 1$ and $\varphi \mid N \equiv \chi$. Let us put $T=$ ind $_{F_{x} \uparrow F} \varphi ; T$ is an irreducible representation of $F$ but, as we are going to show, not CCR. To this end we prove that the $\operatorname{kernel} \operatorname{ker}(T)$ of $T$, considered as a representation of $C^{*}(F)$, is not a maximal 2-sided ideal. We have, in fact,

$$
T=a_{n} T=\operatorname{ind}_{F_{x} \uparrow F}\left(a_{n} \varphi\right) \text {, }
$$

and $\left\{a_{n} \varphi\right\}$ converges to the unit character $\varphi_{0}$ of $X\left(F_{X}\right)$. Putting $Z=$ 
$\operatorname{ind}_{F_{\chi} \uparrow F} \varphi_{0}$, by virtue of the continuity of induction we conclude that $\operatorname{ker}(Z)$ $\supseteq \operatorname{ker}(T)$ and thus also $\operatorname{ker}(Z \mid N) \supseteq \operatorname{ker}(T \mid N)$ (cf. e.g. [20, p. 90, top]). But this is impossible since $Z \mid N$ is the trivial representation and $T \mid N$ decomposes to the orbit $F \chi \subset \hat{N}=X(N)$ and $\chi \neq 1$.

$\operatorname{Ad}(B)$ We recall that here we assume that $r=(R)$ and $g / r$ is not compact. We shall now use also the hypothesis that $g$ has no semisimple factors $(\mathrm{g}=$ n.s.f. $)$.

(a) We show first the existence of a factor algebra $f$ of $g$ having the quoted properties and the nilradical of which is a minimal ideal. (1) By what we saw in $(A)(a)(1)$, since $\mathfrak{g}=$ n.s.f., if $\mathfrak{h}$ is a Levi section, it acts faithfully on $\mathfrak{n} /[\mathfrak{n}, \mathfrak{n}]$, and thus we can assume from the beginning that $\mathfrak{n}$ is abelian. (2) Since $g / r$ is not compact, $\mathfrak{h}$ contains a subalgebra $\mathfrak{h}_{1}$ which is isomorphic to the Lie algebra of all $2 \times 2$ matrices with real coefficients and trace zero. (3) There is a finite set $\mathcal{G}$ of pairwise inequivalent irreducible representations of $\mathfrak{h}$ such that $\mathfrak{n}$ is a direct sum $\Sigma_{\gamma \in \mathcal{G}}$ where $V_{\gamma}$ is a $\gamma$ pure $\mathfrak{h}$ module. Since $\mathfrak{n}$ is abelian, $\mathfrak{g}$ acts on $\mathfrak{n}$ as does $\mathfrak{g} / \mathfrak{n}$ and hence (cf. [3, Proposition 1, p. 41] $V_{\gamma}$ is a $g$ ideal. (4) Let us suppose that the restriction of $\alpha \in \mathcal{G}$, say, to $\mathfrak{h}_{1}$ is nontrivial. Dividing by $\Sigma_{\gamma \neq \alpha} V_{\gamma}$ we can assume that the action of $\mathfrak{h}$ on $\mathfrak{n}$ gives rise to only one nontrivial irreducible representation, the kernel of which does not contain $\mathfrak{h}_{1}$. (5) Imitating the procedure of $(A)(a)$ we can construct a factor algebra $f$ with the properties claimed at the start (note that one may have to divide by a semisimple direct factor). We continue to denote by $\mathfrak{h}$ a Levi section and assume that $\mathfrak{h}_{1} \subset \mathfrak{h}$ is as above in (2).

(b) We claim that $F=\exp (\mathfrak{f})$ is of type I. To this end, as in (A), it is enough to show that $\mathfrak{f}=(a)$. Let $r$ and $\mathfrak{n}$ be, respectively, the radical and nilradical of $\mathfrak{f}$. Since $\mathfrak{n}$ is minimal, the restriction of $\operatorname{ad}(\mathfrak{r})$ to $\mathfrak{n}$ is composed of semisimple endomorphisms. Also, the only nonzero root of $r$ is purely imaginary. Hence $r=(a)$ and thus also $f=(a)$.

(c) We show finally that $F$ admits an irreducible unitary representation which is not CCR. To this end we recall first that there is an $l \neq 0$ in $\mathfrak{h}_{1}$ such that $\operatorname{ad}(l) \mid \mathfrak{n}$ has integral eigenvalues (cf. [22, 4.3.10, Proposition, p. 89]). Let $0 \neq f \in \mathfrak{n}^{\prime}$ be such that $l f=k l(k>0$, say) and suppose that $\chi \in X(N)$ satisfies $d \chi=i$. If $h(t) \equiv \exp (t l)$, we have $\lim _{t \rightarrow+\infty} h(t) \chi=\chi_{0}$ (= unit of $X(N))$. Let us put $H=\exp (\mathfrak{h}), A=\exp (\mathfrak{a})$ and $K=H A \subset F ; F$ is the direct product of the closed subgroups $K$ and $N$. We have also $F_{\chi}=K_{\chi} \cdot N$, and there is a $\varphi \in X\left(F_{\chi}\right)$ such that $\varphi \mid K_{\chi} \equiv 1$ and $\varphi \mid N \equiv \chi$. Observing that $F_{\chi}$ is normalized by $\{h(t) ; t \in \mathrm{R}\}$, we can show, similarly as in $(\mathrm{A})(\mathrm{c})$, that $T=\operatorname{ind}_{F_{x} \uparrow F} \varphi$ is an irreducible representation which is not CCR.

2.2. Lemma 24. Assume that $\mathrm{g}$ has no semisimple factors. Putting $G=\exp (\mathrm{g})$ and denoting by $\mathfrak{r}$ the radical of $\mathfrak{g}$ let us consider the following properties. (i) If $O \in W(G) / G$ and $\operatorname{dim}(O)>0$, then the closure $\bar{O}$ of $O$ does not contain the 
neutral element of $g^{\prime}$; (ii) $r$ is of type $R$ and $g / r$ is compact. We claim that (1) (i) implies (ii); (2) if (ii) holds and $O \in \mathrm{g}^{\prime} / G, \bar{O}$ is a union of $G$ orbits of the same dimension; (3) if (i) or (ii) holds, and $O \in \mathrm{g}^{\prime} / G$ is open in its closure, then $\boldsymbol{O}$ is closed.

Proof. Ad(1) If (ii) does not hold, then either (a) $g / \mathfrak{r}$ is not compact or (b) $r$ is nor of type $R$.

(a) We write $\mathfrak{h}$ for the semisimple algebra $\mathfrak{g} / \mathfrak{r}$ and set $H=\exp (\mathfrak{h})$. There is a canonical identification, by aid of the Killing form, between $\mathfrak{h}$ and $\mathfrak{h}^{\prime}$ considered as $H$ modules. Since $\mathfrak{g}$ acts as $\mathfrak{g} / \mathfrak{r}=\mathfrak{h}$ on the orthogonal complement $\mathfrak{r}^{\perp}$ of $\mathfrak{r}$ in $\mathfrak{g}^{\prime}$, there is a similar identification between $\mathfrak{h}^{\prime}$ and $\mathbf{r}^{\perp} \subset \mathfrak{g}^{\prime}$. Therefore, to obtain a contradiction to our assumption it is enough to show that there is a $l \in \mathfrak{h}$ such that $\operatorname{dim}(H l)>0$ and $\overline{H l}$ contains the neutral element of $\mathfrak{h}$. Since $\mathfrak{h}$ is noncompact, it contains a subalgebra $\mathfrak{h}_{1}$ isomorphic to the Lie algebra of all $2 \times 2$ real matrices with trace zero. $\mathfrak{h}_{1}$ admits a basis $\{h, l, k\}$ such that $[h, l]=2 l,[h, k]=-2 k$ and $[l, k]=h$. Since $\exp (t h) l \equiv \exp (2 t) \cdot l$, we have $\operatorname{dim}(H l)>0$ and $\overline{H l}$ contains the origin. In this fashion it will suffice to prove that $l \in W(H)$. Since $\mathfrak{h}$ is semisimple, to this end it is enough to exhibit a $\chi_{1} \in X\left(\left(H_{l}\right)_{0}\right)$ such that $d \chi_{1}=i\left(l \mid \mathfrak{h}_{1}\right)$ (cf. II.2.1). But this point is settled at once by observing that $h \mid \mathfrak{h}_{1}=0$ since: (1) $l$ is a nilpotent element of $\mathfrak{h}$; (2) $\mathfrak{h} l$ is just the centralizer of $l$ in $\mathfrak{h}$.

(b) Now we assume that $r \neq(R)$. By what we saw in the proof of Lemma 23 (cf. loc. cit. (A)(a) end) there is a $g$ ideal $r_{0}$ in $r$ such that, putting $f=g / r_{0}$ the radical of $f$ is not of type $R$ and its nilradical $\mathrm{m}$, say, is minimal. We write $F=\exp (f)$ and observe that it is enough to establish the existence of a $g \in W(F)$ such that $\overline{F g}$ contains the zero element of $f^{\prime}$. In fact, let $h$ be the corresponding element in $r_{0}^{\perp} \subset g^{\prime}$; it is enough then to show that $h \in W(G)$. We denote by $\mathfrak{n}$ the nilradical of $\mathfrak{g}$ and put $f=h \mid \mathfrak{n}$. Then $\mathfrak{n}_{f}=\mathfrak{n}$. In fact, since $\mathfrak{m}$ is abelian in $\mathfrak{f}$, and $\mathfrak{n} /\left(\mathfrak{n} \cap \mathfrak{r}_{0}\right) \subseteq \mathfrak{m}$, we have $[\mathfrak{n}, \mathfrak{n}] \subset \mathfrak{r}_{0}$. In this manner $h \in W(G)$. We can write $\mathfrak{f}=\mathfrak{h}+\mathfrak{a}+\mathfrak{m}$, where $\mathfrak{h}$ is a Levi section, $\mathfrak{a}$ an abelian subalgebra of dimension $\langle 2$, and $[\mathfrak{h}, a]=0$. Let us put $A=$ $\exp (a)$. Since the radical of $f$ is not of type $R$, there is a $0 \neq f \in \mathfrak{m}^{\prime}$ such that $\overline{A f}$ contains zero. Writing $\mathfrak{b}=\mathfrak{h}+\mathfrak{a}$, we define $g \in \mathfrak{f}^{\prime}$ by $g|\mathfrak{b}=0, g| \mathfrak{m}=f$. Since $\mathfrak{f}^{\prime}=\mathfrak{b}^{\perp}+\mathfrak{m}^{\perp}$, the right-hand side being an $A$ invariant direct sum, $\overline{A g}$, too, contains the neutral element of $f^{\prime}$. In this manner to complete our proof, it suffices to establish that $g \in W(F)$. Since $m$ is abelian, it is enough to show the existence of $\chi_{g} \in X\left(\left(F_{g}\right)_{0}\right)$ such that $d \chi_{g}=i\left(g \mid f_{g}\right)$. Putting $B=\exp (\mathfrak{b})$ and $M=\exp (\mathfrak{m})$, we have $F=B M$ and $F_{f}=B_{f} M$. Since evidently $B_{f} \subset F_{g}$, we conclude that $F_{g}=B_{f} M_{g}$ and $\left(F_{g}\right)_{0}=\left(B_{f}\right)_{0} \cdot M_{g}$. This being so, we can take for $\chi_{g}$ the extension of $\chi_{f} \mid M_{g}$ to $\left(F_{g}\right)_{0}$ which is identically one on $\left(B_{f}\right)_{0}$.

$\operatorname{Ad}(2)$ Let $\mathfrak{L}$ be the connected component of the smallest linear algebraic group of endomorphisms of $g$ containing $\operatorname{Ad}(G)$. By virtue of assumption (ii), 
$\mathfrak{Z}$ is a semidirect product of a compact group with a connected unipotent group. Hence any orbit, under the contragredient action. of $\mathfrak{L}$ on $g^{\prime}$ is closed. In this fashion, if $O=G g$ we have $\bar{O} \subset \mathfrak{Q}$. Since $\mathfrak{L}$ contains $\operatorname{Ad}(G)$ as an invariant subgroup, its action on $g^{\prime}$ preserves $G$ orbits; therefore $\bar{O}$ is the union of $G$ orbits of the same dimension.

Ad(3) Let us put again $b=[\mathfrak{g}, g]+\mathfrak{n}$, where $\mathfrak{n}$ is the nilradical of $\mathfrak{g}$, and $L=\exp (\delta)$ (cf. II.2.4). $\&$ contains $\operatorname{Ad}(L)$ as a closed invariant subgroup such that $\mathbb{L} / \operatorname{Ad}(L)$ is abelian. Assume that $O=G g$ is open in its closure. Reasoning as in Lemma 15, with $\mathfrak{R}$ and $\operatorname{Ad}(L)$ in place of $P$ and $L$ loc. cit., we conclude that $\& g / G$ is a $T_{1}$ space. But then $O=\bar{O} \cap \& g=\bar{O}$. Q.E.D.

We summarize the previous results in

Proposmion 3. Suppose that $\mathrm{g}$ does not have semisimple factors, and let $G$ be the corresponding connected and simply connected Lie group. Then the following properties are equivalent: (i) $\operatorname{Prim}(G)$ is a $T_{1}$ space; (ii) any normal representation is generalized completely continuous; (iii) any irreducible normal representation is completely continuous; (iv) the radical is cocompact and its roots are purely imaginary; (v) if $O$ is a $G$ orbit of positive dimension in $W(G)$, its closure does not contain the neutral element of the vector space $g^{\prime}$.

Proof. (i) $\Rightarrow$ (ii) Our assumption says that any primitive ideal in $C^{*}(G)$ is maximal. On the other hand, the kernel of any factor representation of $C^{*}(G)$ is primitive. Hence the desired conclusion follows from Lemma 21. (ii) $\Rightarrow$ (iii) Trivial. (iii) $\Rightarrow$ (iv) Cf. Lemma 23. (iv) $\Rightarrow$ (i) Cf. $[14$, §4]. (iv) $\Rightarrow$ (v) This is clearly implied by Lemma 24 . Q.E.D.

3. As already mentioned (cf. 2.1) any Lie algebra admits a unique representation as a direct product of a semisimple algebra and of an n.s.f. algebra ( $g_{1}$ say). We shall call the subgroup, determined by $g_{1}$, of $G=\exp (g)$ (A.4) the n.s.f. component of the latter. For the definition of $i(O)(O \in$ $W(G) / G)$ cf. II.4.4.

THEOREM 2. Suppose that $G$ is a connected and simply connected Lie group with the Lie algebra g. Then (1) $G$ is CCR if and only if so is its component with no semisimple factors. (2) Assume that $G$ has no semisimple factors. Then it is $C C R$ if and only if any $G$ orbit $O$ in $W(G)$ is closed and $i(O)<+\infty$.

Proof. Ad(1) Since $G$ is a direct product of its n.s.f. component and of a connected semisimple group, to prove our point it suffices to recall that any group of the latter kind is CCR (as follows e.g. from [10, Theorem 5, p. 248]).

$\operatorname{Ad}(2)(a)$ We suppose first that $G$ is a CCR group. Then by Proposition 3 ((iii) $\Rightarrow$ (iv)), in particular, the radical is cocompact. Since now $G$ is of type $I$, we infer from Theorem 1 that any $G$ orbit $O$ in $W(G)$ is locally closed and that $i(O)<+\infty$. Hence it suffices to show that $O$ is closed. By the quoted 
implication of Proposition 3, the radical of $g$ is also of type $R$. Thus the desired conclusion follows from Lemma 24(3).

(b) If any $O \in W(G) / G$ is closed and $i(O)<+\infty$, by Proposition 3 $((v) \Rightarrow(i v))$ and Theorem $1, G$ is of type I. If so, any irreducible unitary representation is normal (cf. $[6,9.1$. Théorème, (i) $\Rightarrow$ (iv), p. 168]). Hence, invoking again Proposition $3((v) \Rightarrow($ iii)) we conclude finally that $G$ is $C C R$. Q.E.D.

COROLLARY. Let $G$ be a connected and simply connected solvable Lie group. Then $G$ is $C C R$ if and only if any $G$ orbit in $g^{\prime}$ is closed and, for any $g \in g^{\prime}$, there is $\chi_{g} \in X\left(G_{g}\right)$ such that $d \chi_{g}=i\left(g \mid g_{g}\right)$.

This follows immediately from Theorem 2 and Corollary II.4.5 by observing that now $W(G)=g^{\prime}$ (cf. II.2.1).

\section{APPENDIX. SOME GENERAL ASSUMPTIONS AND NOTATIONAL CONVENTIONS}

(1) If $G$ is a group acting on a set $S, p$ fixed in $S, G_{p}$ stands for the stabilizer of $p$ in $G$. If $G$ is a Lie group with the Lie algebra $g$, and if $G_{p}$ is closed, $g_{p}$ stands for the corresponding subalgebra of $\mathfrak{g} . \mathfrak{g}$ and its ideals will be considered as $G$ modules with respect to the adjoint representation and their duals under the corresponding contragredient action.

(2) Given a unitary representation $V$, we denote by $H(V)$ the space of representation. All Hilbert spaces considered are assumed separable. We shall indicate by $U \sim V$ the unitary equivalence of the unitary representations $U$ and $V$. Often the same symbol will be used for a concrete unitary representation and its unitary equivalence class. Accordingly, we shall frequently write $U=V$ in place of $U \sim V$.

(3) If $G$ is a group, $H$ a subgroup of $G, \rho$ a representation of $H$ and $\alpha \in \operatorname{Aut}(G), \alpha \rho$ will stand for the representation of $\alpha(H)$ defined by $(\alpha \rho)(\alpha(x)) \equiv \rho(x)(x \in H)$.

(4) If $g$ is a Lie algebra, unless otherwise specified, $\exp (g)$ will stand for a corresponding connected and simply connected Lie group. If $\mathfrak{h}$ is a subalgebra of $\mathfrak{g}$, and some connected Lie group, belonging to $\mathfrak{g}$, is specified by the context, $\exp (\mathfrak{h})$ denotes its analytic subgroup determined by $\mathfrak{h}$.

(5) Given a topological group $G$, we denote by $X(G)$ the set of all continuous homomorphisms of $G$ into the circle group. If $\Gamma$ is some subgroup of $G, Y(G / \Gamma)$ will stand for the subgroup of elements, trivial on $\Gamma$, of $X(G)$.

(6) $G_{0}$ denotes the connected component of the identity in $G$.

(7) Given a group or algebra $A, A^{\natural}$ stands for its center.

(8) Given a unitary representation $T$ of some group, $R(T)$ will denote the von Neumann algebra it generates. 


\section{BIBLIOGRAPHY}

1. L. Auslander and B. Kostant, Polarization and unitary representations of solvable Lie groups, Invent. Math. 14 (1971), 255-354. MR 45 \#2092.

2. L. Auslander and C. C. Moore, Unitary representations of solvable Lie groups, Mem. Amer. Math. Soc. No. 62 (1966), MR 34 \#7723.

3. N. Bourbaki, Eléménts de mathématique. XXVI. Chap. 1: Algèbres de Lie, Actualités Sci. Indust., no. 1285, Hermann, Paris, 1960. MR 24 \# A2641.

4. I. D. Brown, Dual topology of a nilpotent Lie group, Ann. Sci. École Norm. Sup. (4) 6 (1973), 407-411. MR 50 \#4813.

5. J. Dixmier, Sur les representations unitaires des groupes de Lie nilpotents. V, Bull. Soc. Math. France 87 (1959), 65-79. MR 22 \#5900a.

6. __ Les $C^{*}$ algèbres et leurs représentations, Cahiers Scientifiques, fasc. 29, GauthierVillars, Paris, 1964. MR 30 \# 1404.

7. Sur la représentation régulière d'un groupe localement compacte connexe, Ann. Sci. École Norm. Sup. (4) 2 (1969), 423-436. MR 41 \#5553.

8. M. Duflo, Sur les extensions des représentations irréductibles des groupes de Lie nilpotents, Ann. Sci. Ecole Norm. Sup. (4) 5 (1972), 71-120. MR 46 \# 1966.

9. J. Glimm, Locally compact transformation groups, Trans. Amer. Math. Soc. 101 (1961), 124-138. MR 25 \# 146.

10. Harish-Chandra, Representations of semisimple Lie groups. III, Trans. Amer. Math. Soc. 76 (1954), 234-253. MR 16, 11.

11. B. Kostant, Quantization and unitary representations, Lecture Notes in Math., vol. 170, Springer-Verlag, Berlin, 1970, pp. 87-208. MR 45 \#3638.

12. R. Lipsman, The CCR property for algebraic groups, Amer. J. Math. 97 (1975), 741-752.

13. G. W. Mackey, Unitary representations of groups extensions. I, Acta Math. 99 (1958), 265-311. MR 20 \#4789.

14. C. C. Moore and J. Rosenberg, Groups with $T_{1}$ primitive ideal spaces, J. Functional Analysis 22 (1976), 204-224.

15. L. Pukaniszky, On the characters and the Plancherel formula of nilpotent groups, J. Functional Analyzis 1 (1967), 255-280. MR 37 \#4236.

16. On the unitary representations of exponential groups, J. Functional Analysis 2 (1968), 73-113. MR 37 \#4205.

17. Characters of algebraic solvable groups, J. Functional Analysis 3 (1969), 435-494. MR 40 \# 1539.

18. 457-608.

, Unitary representations of solvable Lie groups, Ann. Sci. Ėcole Norm. Sup. 4 (1971),

19. __, Action of algebraic groups of automorphisms on the dual of a class of type I groups, Ann. Sci. Ecole Norm. Sup. (4) 5 (1972), 379-395. MR 48 \#466.

20. __, Characters of connected Lie groups, Acta Math. 133 (1974), 81-137.

21. Lie groups with completely continuous representations, Bull. Amer. Math. Soc. 81 (1975), 1061-1063.

22. N. Wallach, Harmonic analysis on homogeneous spaces, Dekker, New York, 1973.

Departmant of Mathematics, University of Pennsyzvania, Philadelphia, Pennsylvania 19174 